NIST Special Publication 1500-9r1

\title{
NIST Big Data Interoperability Framework: Volume 8, Reference Architecture Interfaces
}

\section{Version 3}

NIST Big Data Public Working Group Definitions and Taxonomies Subgroup 


\title{
NIST Special Publication 1500-9r1
}

\section{NIST Big Data Interoperability Framework: Volume 8, Reference Architecture Interfaces}

Version 3

\author{
NIST Big Data Public Working Group \\ Definitions and Taxonomies Subgroup \\ Information Technology Laboratory \\ National Institute of Standards and Technology \\ Gaithersburg, MD 20899
}

This publication is available free of charge from:

https://doi.org/10.6028/NIST.SP.1500-9r1

October 2019

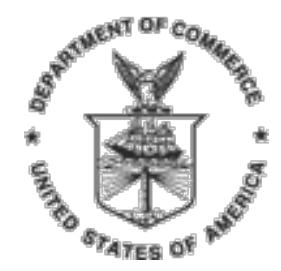

U.S. Department of Commerce Wilbur L. Ross, Jr., Secretary

National Institute of Standards and Technology Walter Copan, NIST Director and Undersecretary of Commerce for Standards and Technology 


\title{
National Institute of Standards and Technology (NIST) Special Publication 1500-9r1 168 pages (October 2019)
}

NIST Special Publication series 1500 is intended to capture external perspectives related to NIST standards, measurement, and testing-related efforts. These external perspectives can come from industry, academia, government, and others. These reports are intended to document external perspectives and do not represent official NIST positions.

Certain commercial entities, equipment, or materials may be identified in this document to describe an experimental procedure or concept adequately. Such identification is not intended to imply recommendation or endorsement by NIST, nor is it intended to imply that the entities, materials, or equipment are necessarily the best available for the purpose.

There may be references in this publication to other publications currently under development by NIST in accordance with its assigned statutory responsibilities. The information in this publication, including concepts and methodologies, may be used by federal agencies even before the completion of such companion publications. Thus, until each publication is completed, current requirements, guidelines, and procedures, where they exist, remain operative. For planning and transition purposes, federal agencies may wish to closely follow the development of these new publications by NIST.

Organizations are encouraged to review all publications during public comment periods and provide feedback to NIST. All NIST publications are available at http://www.nist.gov/publication-portal.cfm.

\section{Copyrights and Permissions}

Official publications of the National Institute of Standards and Technology are not subject to copyright in the United States. Foreign rights are reserved. Questions concerning the possibility of copyrights in foreign countries should be referred to the Office of Chief Counsel at NIST via email to nistcounsel@nist.gov.

\section{Comments on this publication may be submitted to Wo Chang}

\author{
National Institute of Standards and Technology \\ Attn: Wo Chang, Information Technology Laboratory \\ 100 Bureau Drive (Mail Stop 8900) Gaithersburg, MD 20899-8930 \\ Email: SP1500comments@nist.gov
}




\section{Reports on Computer Systems Technology}

The Information Technology Laboratory (ITL) at NIST promotes the U.S. economy and public welfare by providing technical leadership for the Nation's measurement and standards infrastructure. ITL develops tests, test methods, reference data, proof of concept implementations, and technical analyses to advance the development and productive use of information technology. ITL's responsibilities include the development of management, administrative, technical, and physical standards and guidelines for the cost-effective security and privacy of other than national security-related information in federal information systems. This document reports on ITL's research, guidance, and outreach efforts in Information Technology and its collaborative activities with industry, government, and academic organizations.

\section{Abstract}

This document summarizes interfaces that are instrumental for the interaction with Clouds, Containers, and High Performance Computing (HPC) systems to manage virtual clusters to support the NIST Big Data Reference Architecture (NBDRA). The REpresentational State Transfer (REST) paradigm is used to define these interfaces, allowing easy integration and adoption by a wide variety of frameworks.

Big Data is a term used to describe extensive datasets, primarily in the characteristics of volume, variety, velocity, and/or variability. While opportunities exist with Big Data, the data characteristics can overwhelm traditional technical approaches, and the growth of data is outpacing scientific and technological advances in data analytics. To advance progress in Big Data, the NIST Big Data Public Working Group (NBD-PWG) is working to develop consensus on important fundamental concepts related to Big Data. The results are reported in the NIST Big Data Interoperability Framework (NBDIF) series of volumes. This volume, Volume 8, uses the work performed by the NBD-PWG to identify objects instrumental for the NIST Big Data Reference Architecture (NBDRA) which is introduced in the NBDIF: Volume 6, Reference Architecture.

\section{Keywords}

Adoption; barriers; implementation; interfaces; market maturity; organizational maturity; project maturity; system modernization. 


\section{Acknowledgements}

This document reflects the contributions and discussions by the membership of the NBD-PWG, cochaired by Wo Chang (NIST ITL), Bob Marcus (ET-Strategies), and Chaitan Baru (San Diego Supercomputer Center; National Science Foundation). For all versions, the Subgroups were led by the following people: Nancy Grady (SAIC), Natasha Balac (San Diego Supercomputer Center), and Eugene Luster (R2AD) for the Definitions and Taxonomies Subgroup; Geoffrey Fox (Indiana University) and Tsegereda Beyene (Cisco Systems) for the Use Cases and Requirements Subgroup; Arnab Roy (Fujitsu), Mark Underwood (Krypton Brothers; Synchrony Financial), and Akhil Manchanda (GE) for the Security and Privacy Subgroup; David Boyd (InCadence Strategic Solutions), Orit Levin (Microsoft), Don Krapohl (Augmented Intelligence), and James Ketner (AT\&T) for the Reference Architecture Subgroup; and Russell Reinsch (Center for Government Interoperability), David Boyd (InCadence Strategic Solutions), Carl Buffington (Vistronix), and Dan McClary (Oracle), for the Standards Roadmap Subgroup.

The editors for this document were the following:

- Version 1: This volume resulted from Stage 2 work and was not part of the Version 1 scope.

- Version 2: Gregor von Laszewski (Indiana University) and Wo Chang (NIST).

- Version3: Gregor von Laszewski (Indiana University) and Wo Chang (NIST).

Laurie Aldape (Energetics Incorporated) and Elizabeth Lennon (NIST) provided editorial assistance across all NBDIF volumes.

NIST SP 1500-9, Draft NIST Big Data Interoperability Framework: Volume 8, Reference Architecture Interfaces, Version 2 has been collaboratively authored by the NBD-PWG. As of the date of publication, there are over six hundred NBD-PWG participants from industry, academia, and government. Federal agency participants include the National Archives and Records Administration (NARA), National Aeronautics and Space Administration (NASA), National Science Foundation (NSF), and the U.S. Departments of Agriculture, Commerce, Defense, Energy, Census, Health and Human Services, Homeland Security, Transportation, Treasury, and Veterans Affairs.

NIST would like to acknowledge the specific contributions ${ }^{1}$ to this volume, during Version 1 , Version 2, and/or Version 3 activities, by the following NBD-PWG members:

Wo Chang

National Institute of Standard and Technology

Geoffrey C. Fox

Indiana University

Pratik Thakkar

Philips

Gregor von Laszewski

Indiana University
Badi Abdhul Wahid

Indiana University

Fugang Wang

Indiana University

Robert C. Whetsel

DISA/NBIS

Alicia Zuniga-Alvarado

Consultant

\footnotetext{
1 “Contributors” are members of the NIST Big Data Public Working Group who dedicated great effort to prepare and gave substantial time on a regular basis to research and development in support of this document.
} 


\section{TABLE OF CONTENTS}

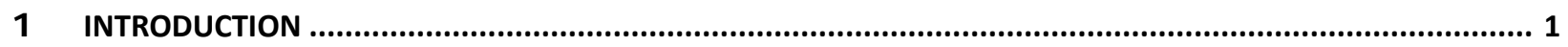

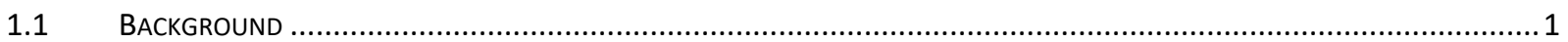

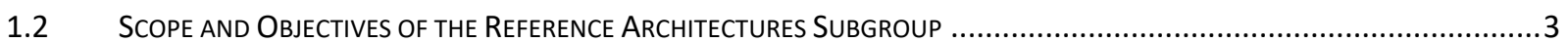

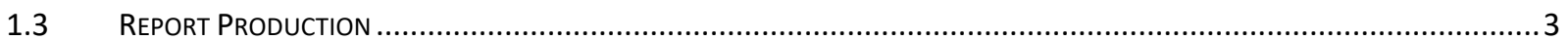

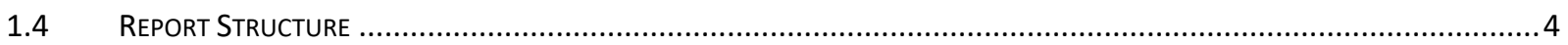

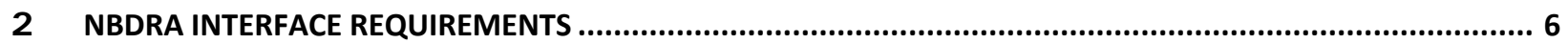

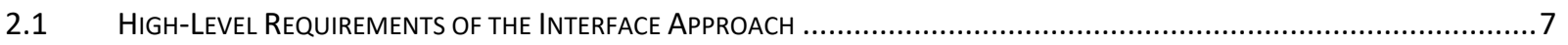

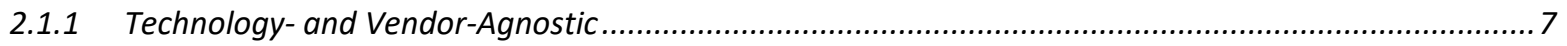

2.1.2 Support of Plug-In Compute Infrastructure................................................................................... 7

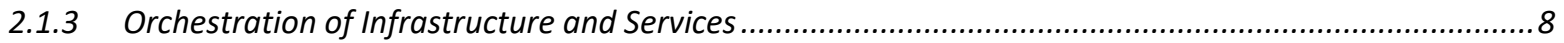

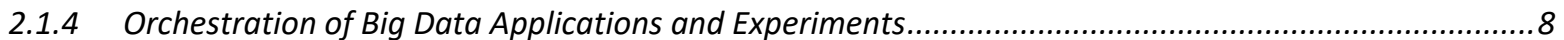

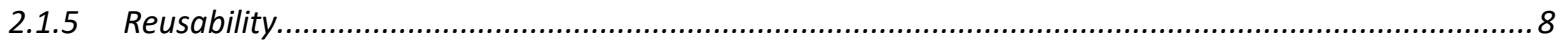

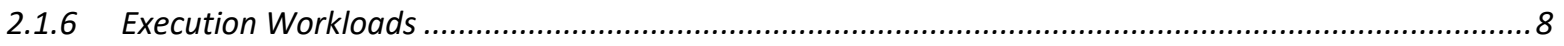

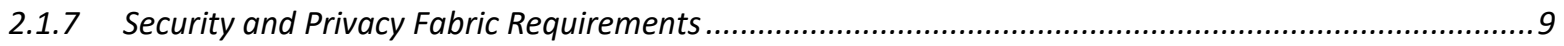

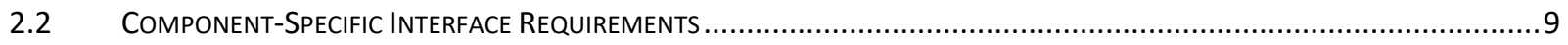

2.2.1 System Orchestrator Interface Requirements..............................................................................

2.2.2 Data Provider Interface Requirements .................................................................................... 10

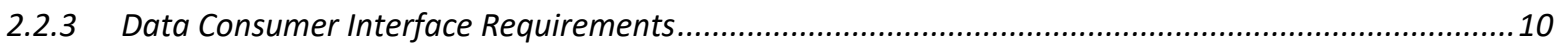

2.2.4 Big Data Application Provider Interface Requirements ................................................................ 10

2.2.5 Big Data Framework Provider Interface Requirements ................................................................12

2.2.6 Big Data Application Provider to Big Data Framework Provider Interface..........................................13

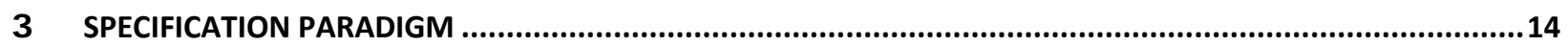

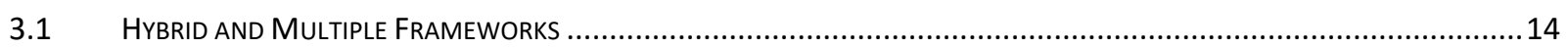

3.2 DESIGN BY RESOURCE-ORIENTED ARCHITECTURE

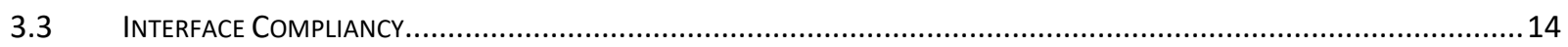

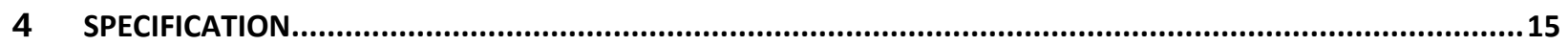

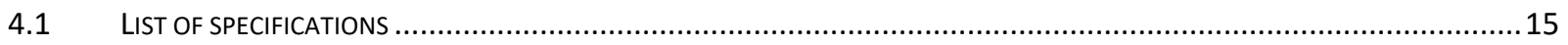

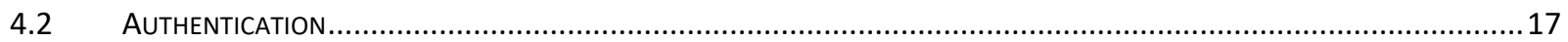

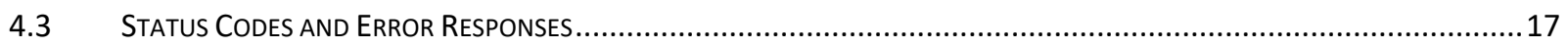

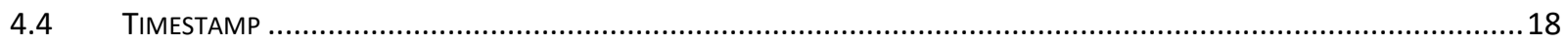

4.4.1 Timestamp

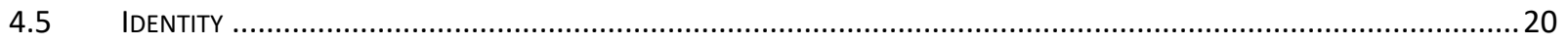

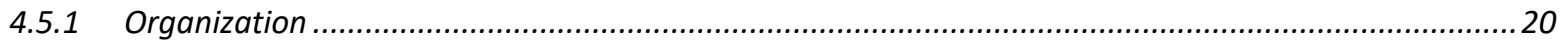

4.5.2 User

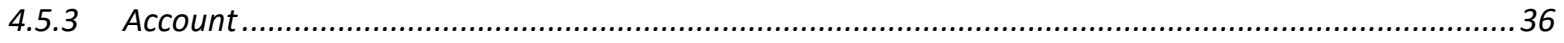

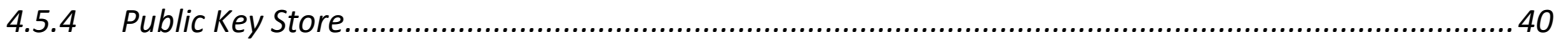

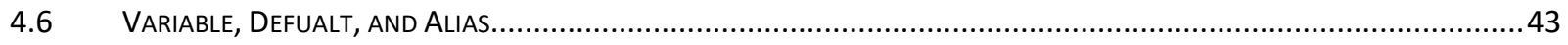

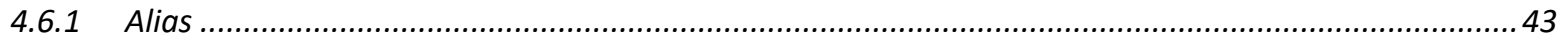

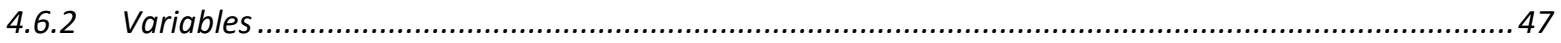

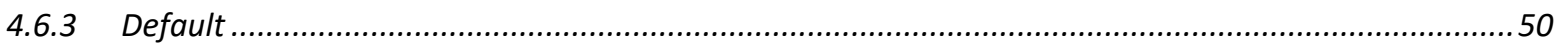

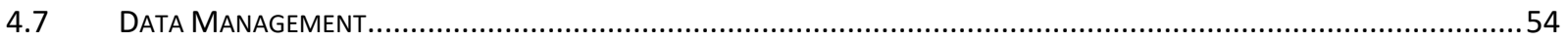

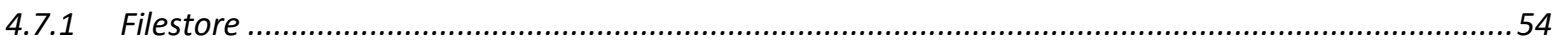

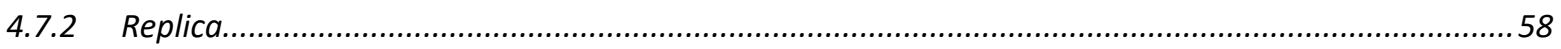


NIST Big DATA INTEROPERABILITY FRAMEWORK: Volume 8, REFERENCE ARCHITECTURE INTERFACES

4.7.3 Database

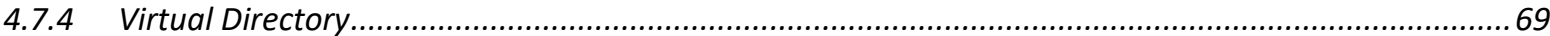

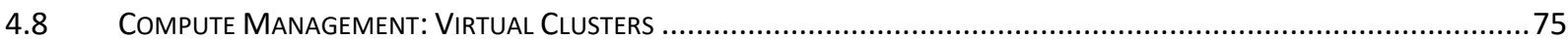

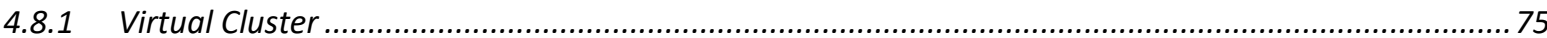

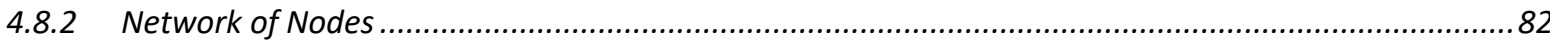

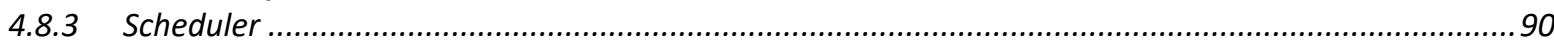

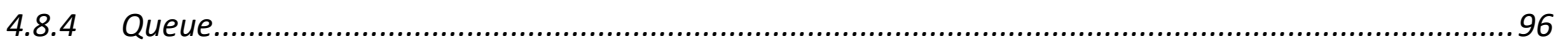

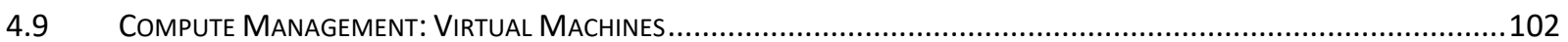

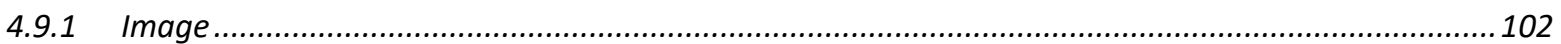

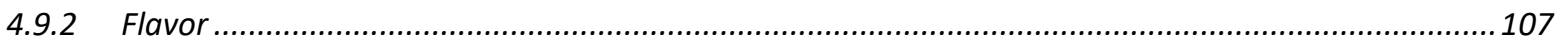

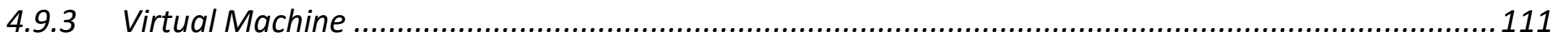

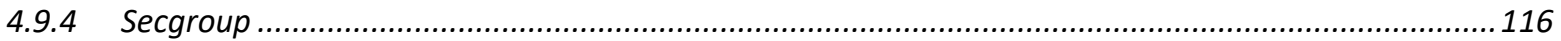

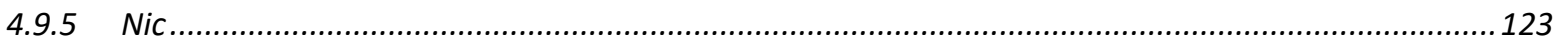

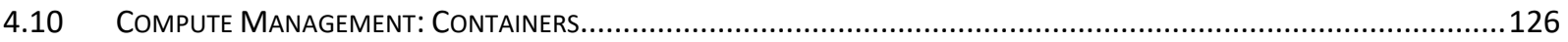

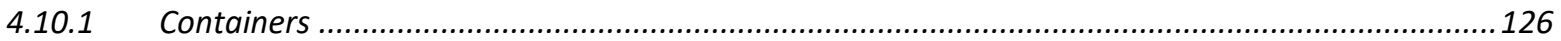

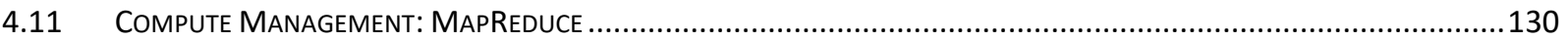

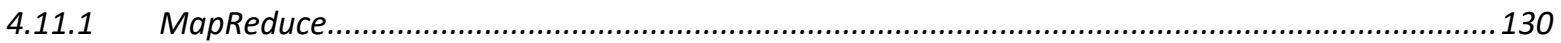

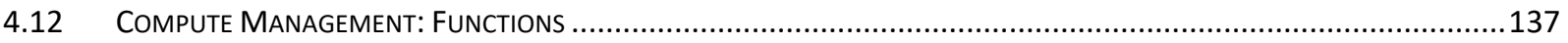

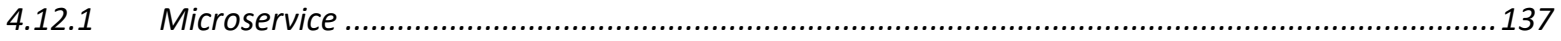

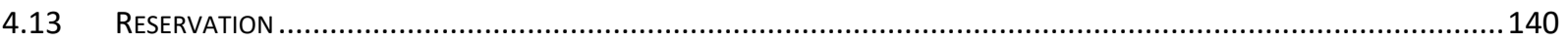

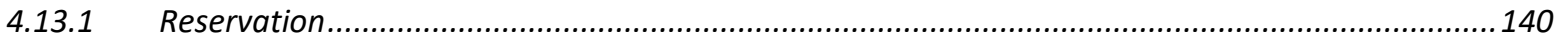

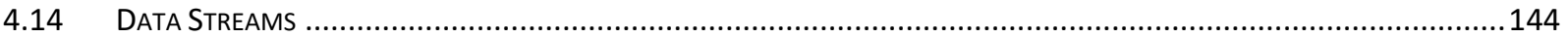

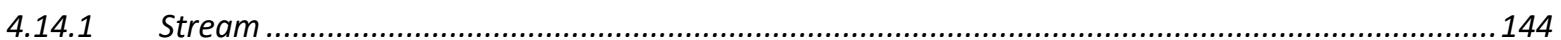

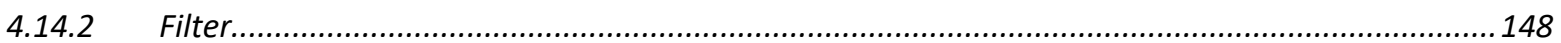

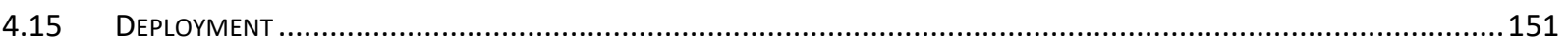

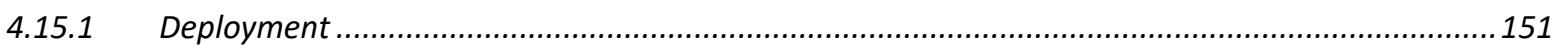

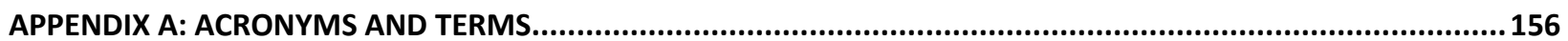

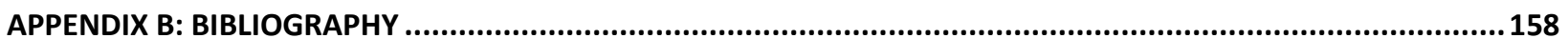

\section{FI GURES}

Figure 1: NBDif Documents Navigation Diagram Provides Content Flow Between Volumes ..........................................5

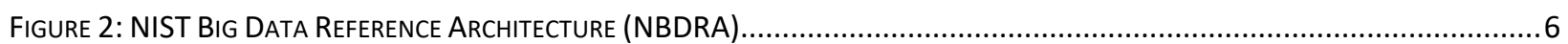

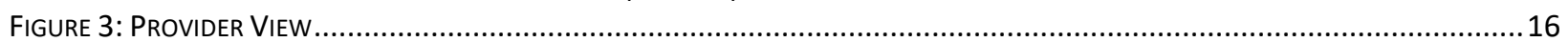

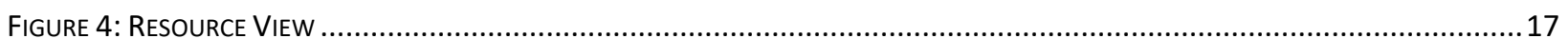

\section{TABLES}

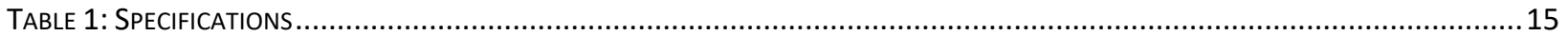

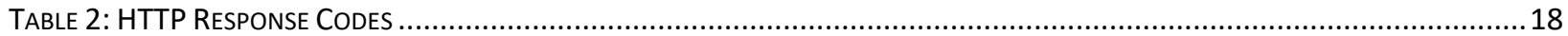




\section{Executive Summary}

The NIST Big Data Interoperability Framework (NBDIF): Volume 8, Reference Architecture Interfaces document was prepared by the NIST Big Data Public Working Group (NBD-PWG) Reference Architecture Subgroup to identify interfaces in support of the NIST Big Data Reference Architecture (NBDRA). The interfaces define resources that are part of the NBDRA. These resources are formulated in OpenAPI 3.0.2 format and can be easily integrated into a REpresentational State Transfer (REST) framework or an object-based framework.

The resources were categorized in groups that are identified by the NBDRA set forth in the NBDIF: Volume 6, Reference Architecture document. While the NBDIF: Volume 3, Use Cases and General Requirements document provides application-oriented high-level use cases, the use cases defined in this document are subsets of them and focus on interface use cases. The interface use cases are not meant to be complete examples, but showcase why a resource has been defined. Hence, the interfaces use cases are only representative and do not encompass the entire spectrum of Big Data usage. All the interfaces were openly discussed in the NBD-PWG [1].

The NBDIF was released in three versions, which correspond to the three stages of the NBD-PWG work. Version 3 (current version) of the NBDIF volumes resulted from Stage 3 work with major emphasis on the validation of the NBDRA Interfaces and content enhancement. Stage 3 work built upon the foundation created during Stage 2 and Stage 1. The current effort documented in this volume reflects concepts developed within the rapidly evolving field of Big Data. The three stages (in reverse order) aim to achieve the following with respect to the NIST Big Data Reference Architecture (NBDRA).

Stage 3: Validate the NBDRA by building Big Data general applications through the general interfaces;

Stage 2: Define general interfaces between the NBDRA components; and

Stage 1: Identify the high-level Big Data reference architecture key components, which are technology-, infrastructure-, and vendor-agnostic.

The NBDIF consists of nine volumes, each of which addresses a specific key topic, resulting from the work of the NBD-PWG. The nine volumes are as follows:

- Volume 1, Definitions [2]

- Volume 2, Taxonomies [3]

- Volume 3, Use Cases and General Requirements [4]

- Volume 4, Security and Privacy [5]

- Volume 5, Architectures White Paper Survey [6]

- Volume 6, Reference Architecture [7]

- Volume 7, Standards Roadmap [8]

- Volume 8, Reference Architecture Interfaces (this volume)

- Volume 9, Adoption and Modernization [9]

During Stage 1, Volumes 1 through 7 were conceptualized, organized, and written. The finalized Version 1 documents can be downloaded from the V1.0 Final Version page of the NBD-PWG website (https://bigdatawg.nist.gov/V1 output docs.php).

During Stage 2, the NBD-PWG developed Version 2 of the NBDIF Version 1 volumes, with the exception of Volume 5, which contained the completed architecture survey work that was used to inform Stage 1 work of the NBD-PWG. The goals of Version 2 were to enhance the Version 1 content, define general interfaces between the NBDRA components by aggregating low-level interactions into high-level 
NIST Big DATA INTEROPERABILITY FRAMEWORK: VOLUME 8, REFERENCE ARCHITECTURE INTERFACES

44 general interfaces, and demonstrate how the NBDRA can be used. As a result of the Stage 2 work, the 45 need for NBDIF Volume 8 and NBDIF Volume 9 was identified and the two new volumes were created.

46 Version 2 of the NBDIF volumes, resulting from Stage 2 work, can be downloaded from the V2.0 Final

47 Version page of the NBD-PWG website (https://bigdatawg.nist.gov/V2 output docs.php).

48 This document is the result of Stage 3 work of the NBD-PWG. Coordination of the group is conducted on 49 the NBD-PWG web page [1].

50 


\section{INTRODUCTION}

\subsection{BACKGROUND}

There is broad agreement among commercial, academic, and government leaders about the potential of Big Data to spark innovation, fuel commerce, and drive progress. Big Data is the common term used to describe the deluge of data in today's networked, digitized, sensor-laden, and information-driven world. The availability of vast data resources carries the potential to answer questions previously out of reach, including the following:

- How can a potential pandemic reliably be detected early enough to intervene?

- Can new materials with advanced properties be predicted before these materials have ever been synthesized?

- How can the current advantage of the attacker over the defender in guarding against cybersecurity threats be reversed?

There is also broad agreement on the ability of Big Data to overwhelm traditional approaches. The growth rates for data volumes, speeds, and complexity are outpacing scientific and technological advances in data analytics, management, transport, and data user spheres.

Despite widespread agreement on the inherent opportunities and current limitations of Big Data, a lack of consensus on some important fundamental questions continues to confuse potential users and stymie progress. These questions include the following:

- How is Big Data defined?

- What attributes define Big Data solutions?

- What is new in Big Data?

- What is the difference between Big Data and bigger data that has been collected for years?

- How is Big Data different from traditional data environments and related applications?

- What are the essential characteristics of Big Data environments?

- How do these environments integrate with currently deployed architectures?

- What are the central scientific, technological, and standardization challenges that need to be addressed to accelerate the deployment of robust, secure Big Data solutions?

Within this context, on March 29, 2012, the White House announced the Big Data Research and Development Initiative [10]. The initiative's goals include helping to accelerate the pace of discovery in science and engineering, strengthening national security, and transforming teaching and learning by improving analysts' ability to extract knowledge and insights from large and complex collections of digital data.

Six federal departments and their agencies announced more than \$200 million in commitments spread across more than 80 projects, which aim to significantly improve the tools and techniques needed to access, organize, and draw conclusions from huge volumes of digital data. The initiative also challenged industry, research universities, and nonprofits to join with the federal government to make the most of the opportunities created by Big Data.

Motivated by the White House initiative and public suggestions, the National Institute of Standards and Technology (NIST) accepted the challenge to stimulate collaboration among industry professionals to further the secure and effective adoption of Big Data. As a result of NIST's Cloud and Big Data Forum held on January 15-17, 2013, there was strong encouragement for NIST to create a public working group 
for the development of a Big Data Standards Roadmap. Forum participants noted that this roadmap should define and prioritize Big Data requirements, including interoperability, portability, reusability, extensibility, data usage, analytics, and technology infrastructure. In doing so, the roadmap would accelerate the adoption of the most secure and effective Big Data techniques and technology.

On June 19, 2013, the NIST Big Data Public Working Group (NBD-PWG) was launched with extensive participation by industry, academia, and government from across the nation. The scope of the NBD-PWG involves forming a community of interests from all sectors-including industry, academia, and government - with the goal of developing consensus on definitions, taxonomies, secure reference architectures, security and privacy, and, from these, a standards roadmap. Such a consensus would create a vendor-neutral, technology- and infrastructure-independent framework that would enable Big Data stakeholders to identify and use the best analytics tools for their processing and visualization requirements on the most suitable computing platform and cluster, while also allowing added value from Big Data service providers.

The NIST Big Data Interoperability Framework (NBDIF) was released in three versions, which correspond to the three stages of the NBD-PWG work. Version 3 (current version) of the NBDIF volumes resulted from Stage 3 work with major emphasis on the validation of the NBDRA Interfaces and content enhancement. Stage 3 work built upon the foundation created during Stage 2 and Stage 1. The current effort documented in this volume reflects concepts developed within the rapidly evolving field of Big Data. The three stages (in reverse order) aim to achieve the following with respect to the NIST Big Data Reference Architecture (NBDRA).

Stage 3: Validate the NBDRA by building Big Data general applications through the general interfaces;

Stage 2: Define general interfaces between the NBDRA components; and

Stage 1: Identify the high-level Big Data reference architecture key components, which are technology-, infrastructure-, and vendor-agnostic.

The NBDIF consists of nine volumes, each of which addresses a specific key topic, resulting from the work of the NBD-PWG. The nine volumes are as follows:

- Volume 1, Definitions [11]

- Volume 2, Taxonomies (this volume)

- Volume 3, Use Cases and General Requirements [12]

- Volume 4, Security and Privacy [13]

- Volume 5, Architectures White Paper Survey [6]

- Volume 6, Reference Architecture [14]

- Volume 7, Standards Roadmap [15]

- Volume 8, Reference Architecture Interfaces [16]

- Volume 9, Adoption and Modernization [17]

During Stage 1, Volumes 1 through 7 were conceptualized, organized, and written. The finalized Version 1 documents can be downloaded from the V1.0 Final Version page of the NBD-PWG website (https://bigdatawg.nist.gov/V1 output docs.php).

During Stage 2, the NBD-PWG developed Version 2 of the NBDIF Version 1 volumes, with the exception of Volume 5, which contained the completed architecture survey work that was used to inform Stage 1 work of the NBD-PWG. The goals of Version 2 were to enhance the Version 1 content, define general interfaces between the NBDRA components by aggregating low-level interactions into high-level general interfaces, and demonstrate how the NBDRA can be used. As a result of the Stage 2 work, the need for NBDIF Volume 8 and NBDIF Volume 9 was identified and the two new volumes were created. Version 2 of the NBDIF volumes, resulting from Stage 2 work, can be downloaded from the V2.0 Final Version page of the NBD-PWG website (https://bigdatawg.nist.gov/V2_output_docs.php). 


\subsection{SCOPE AND OBJECTIVES OF THE REFERENCE ARCHITECTURES SUBGROUP}

Reference architectures provide "an authoritative source of information about a specific subject area that guides and constrains the instantiations of multiple architectures and solutions” [18]. Reference architectures generally serve as a foundation for solution architectures and may also be used for comparison and alignment of instantiations of architectures and solutions.

The goal of the NBD-PWG Reference Architecture Subgroup is to develop an open reference architecture for Big Data that achieves the following objectives:

- Provides a common language for the various stakeholders;

- Encourages adherence to common standards, specifications, and patterns;

- Provides consistent methods for implementation of technology to solve similar problem sets;

- Illustrates and improves understanding of the various Big Data components, processes, and systems, in the context of a vendor- and technology-agnostic Big Data conceptual model;

- Provides a technical reference for U.S. government departments, agencies, and other consumers to understand, discuss, categorize, and compare Big Data solutions; and

- Facilitates analysis of candidate standards for interoperability, portability, reusability, and extendibility.

The NBDRA is a high-level conceptual model crafted to serve as a tool to facilitate open discussion of the requirements, design structures, and operations inherent in Big Data. The NBDRA is intended to facilitate the understanding of the operational intricacies in Big Data. It does not represent the system architecture of a specific Big Data system, but rather is a tool for describing, discussing, and developing systemspecific architectures using a common framework of reference. The model is not tied to any specific vendor products, services, or reference implementation, nor does it define prescriptive solutions that inhibit innovation.

The NBDRA does not address the following:

- Detailed specifications for any organization's operational systems;

- Detailed specifications of information exchanges or services; and

- Recommendations or standards for integration of infrastructure products.

The goals of the Subgroup were realized throughout the three planned phases of the NBD-PWG work, as outlined in Section 1.1.

\subsection{REPORT PRODUCTION}

The NBDIF: Volume 8, References Architecture Interfaces is one of nine volumes, whose overall aims are to define and prioritize Big Data requirements, including interoperability, portability, reusability, extensibility, data usage, analytic techniques, and technology infrastructure to support secure and effective adoption of Big Data. The overall goals of this volume are to define and specify interfaces to implement the Big Data Reference Architecture. This volume arose from discussions during the weekly NBD-PWG conference calls. Topics included in this volume began to take form in Phase 2 of the NBDPWG work. During the discussions, the NBD-PWG identified the need to specify a variety of interfaces.

Phase 3 work, which built upon the groundwork developed during Phase 2, included an early specification based on resource object specifications that provided a simplified version of an API interface design.

The following is a list of milestone releases for this volume: 
- Version 2.1: A previous volume used the definitions of the schema based on examples only. It was easier to read but only included the definition of the resources and not the interaction with the resources. This volume was in place until June 2018.

- Version 2.2: This version was significantly changed and used OpenAPI 2.0 to specify the interfaces between the various services and components.

- Version 3.1.1: The version includes significant improvements of the object specifications but are still using OpenAPI 2.0.

- Version 3.2.0: All specifications were updated to OpenAPI 3.0.2. Significant updates were done to a number of specifications.

\subsection{REPORT STRUCTURE}

To enable interoperability between the NBDRA components, a list of well-defined NBDRA interfaces is needed. These interfaces are documented in this volume. To introduce them, the NBDRA structure will be followed, focusing on interfaces that allow bootstrapping of the NBDRA. The document begins with a summary of requirements that will be integrated into our specifications. Subsequently, each section will introduce a number of objects that build the core of the interface addressing a specific aspect of the NBDRA. A selected number of interface use cases will be showcased to outline how the specific interface can be used in a reference implementation of the NBDRA. Validation of this approach can be achieved while applying it to the application use cases that have been gathered in the NBDIF: Volume 3, Use Cases and Requirements document. These application use cases have considerably contributed towards the design of the NBDRA. Hence the expectation is that the interfaces can be used to help (1) implement a Big Data architecture for a specific use case; and (2) achieve the proper implementation. This approach can facilitate subsequent analysis and comparison of the use cases.

The organization of this document roughly corresponds to the process used by the NBD-PWG to develop the interfaces. Following the introductory material presented in Section 1, the remainder of this document is organized as follows:

- Section 2 presents the interface requirements;

- Section 3 presents the specification paradigm that is used; and

- Section 4 presents several objects grouped by functional use, with a summary table of selected proposed objects in Section 4.1.

While each NBDIF volume was created with a specific focus within Big Data, all volumes are interconnected. During the creation of the volumes, information from some volumes was used as input for other volumes. Broad topics (e.g., definition, architecture) may be discussed in several volumes with each discussion circumscribed by the volume's particular focus. Arrows shown in Figure 1 indicate the main flow of information input and/or output from the volumes. Volumes 2, 3, and 5 (blue circles) are essentially standalone documents that provide output to other volumes (e.g., to Volume 6). These volumes contain the initial situational awareness research. During the creation of Volumes 4, 7, 8, and 9 (green circles), input from other volumes was used. The development of these volumes took into account work on the other volumes. Volumes 1 and 6 (red circles) were developed using the initial situational awareness research and continued to be modified based on work in other volumes. The information from these volumes was also used as input to the volumes in the green circles. 


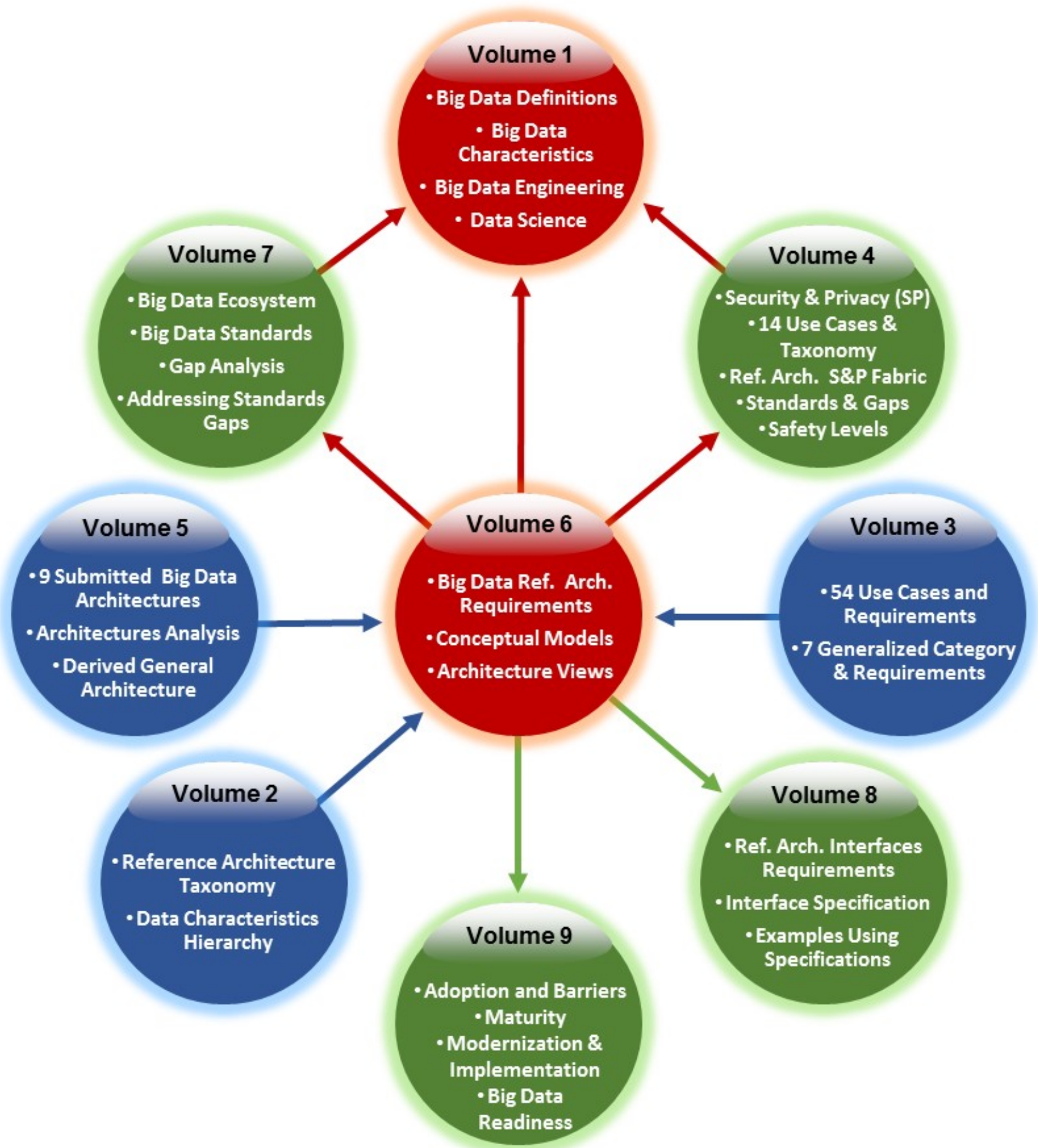

Figure 1: NBDIF Documents Navigation Diagram Provides Content Flow Between Volumes 


\section{NBDRA INTERFACE REQUIREMENTS}

The development of a Big Data reference architecture requires a thorough understanding of current techniques, issues, and concerns. To this end, the NBD-PWG collected use cases to gain an understanding of current applications of Big Data, conducted a survey of reference architectures to understand commonalities within Big Data architectures in use, developed a taxonomy to understand and organize the information collected, and reviewed existing technologies and trends relevant to Big Data. The results of these NBD-PWG activities were used in the development of the NBDRA (Figure 2) and the interfaces presented herein. Detailed descriptions of these activities can be found in the other volumes of the NBDIF.

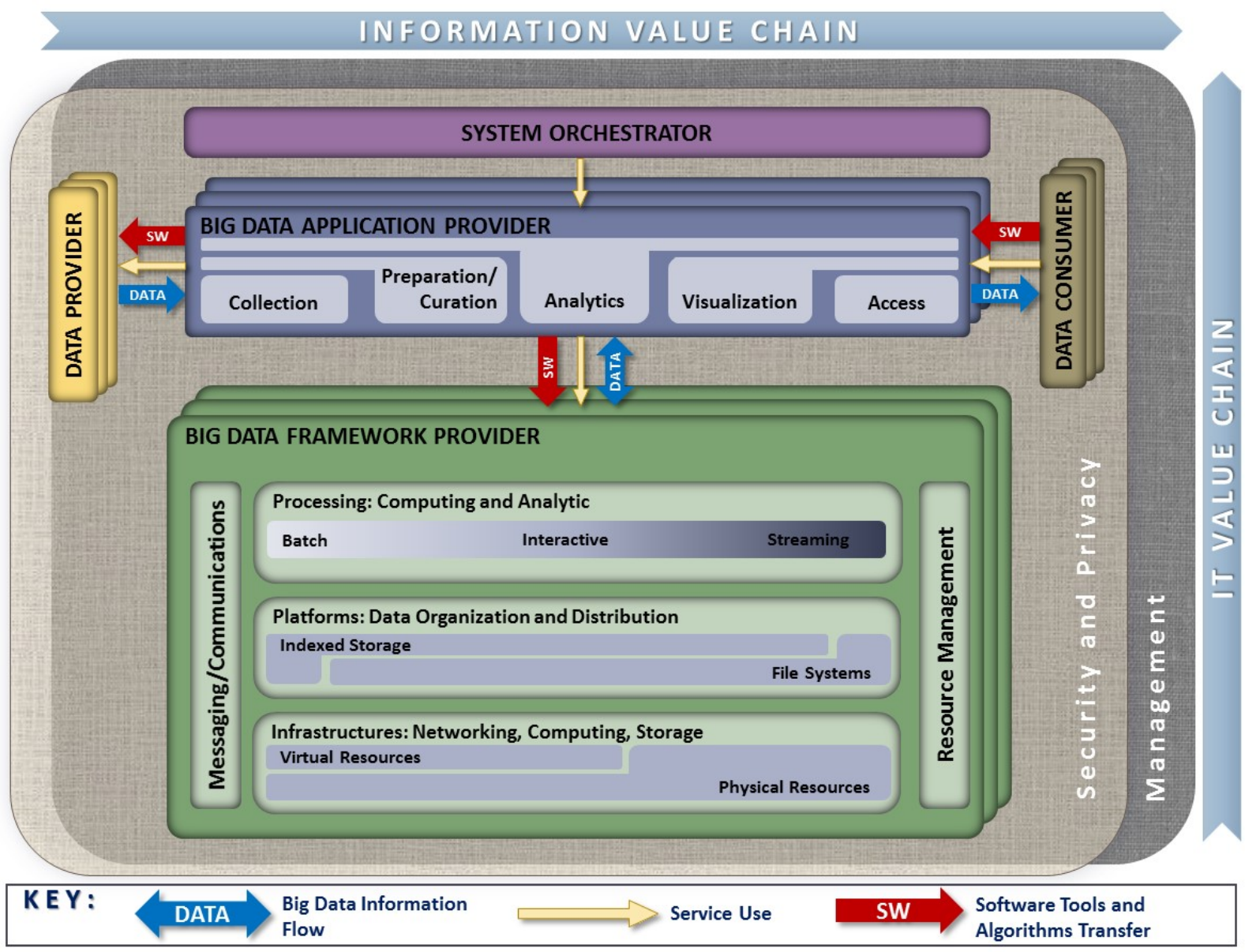

Figure 2: NIST Big Data Reference Architecture (NBDRA)

This vendor-neutral, technology- and infrastructure-agnostic conceptual model, the NBDRA, is shown in Figure 2 and represents a Big Data system composed of five logical functional components connected by interoperability interfaces (i.e., services). Two fabrics envelop the components, representing the interwoven nature of management and security and privacy with all five of the components. These two fabrics provide services and functionality to the five main roles in the areas specific to Big Data and are crucial to any Big Data solution. Note: None of the terminology or diagrams in these documents is 
242

243

244

245

246

247

248

249

250

251

252

253

254

255

256

257

258

259

260

261

262

263

264

265

266

267

268

269

270

271

272

273

274

275

276

277

278

279

280

281

282

intended to be normative or to imply any business or deployment model. The terms provider and consumer as used are descriptive of general roles and are meant to be informative in nature.

The NBDRA is organized around five major roles and multiple sub-roles aligned along two axes representing the two Big Data value chains: the Information Value (horizontal axis) and the Information Technology (IT; vertical axis). Along the Information Value axis, the value is created by data collection, integration, analysis, and applying the results following the value chain. Along the IT axis, the value is created by providing networking, infrastructure, platforms, application tools, and other IT services for hosting of and operating the Big Data in support of required data applications. At the intersection of both axes is the Big Data Application Provider role, indicating that data analytics and its implementation provide the value to Big Data stakeholders in both value chains. The term provider as part of the Big Data Application Provider and Big Data Framework Provider is there to indicate that those roles provide or implement specific activities and functions within the system. It does not designate a service model or business entity.

The DATA arrows in Figure 2 show the flow of data between the system's main roles. Data flows between the roles either physically (i.e., by value) or by providing its location and the means to access it (i.e., by reference). The SW arrows show transfer of software tools for processing of Big Data in situ. The Service Use arrows represent software programmable interfaces. While the main focus of the NBDRA is to represent the run-time environment, all three types of communications or transactions can happen in the configuration phase as well. Manual agreements (e.g., service-level agreements) and human interactions that may exist throughout the system are not shown in the NBDRA.

Detailed information on the NBDRA conceptual model is presented in the NBDIF: Volume 6, Reference Architecture document.

Prior to outlining the specific interfaces, the general requirements are introduced and the interfaces are defined in the following sections.

\subsection{HIGH-LEVEL REQUIREMENTS OF THE INTERFACE APPROACH}

This section focuses on the high-level requirements of the interface approach that are needed to implement the reference architecture depicted in Figure 2.

\subsubsection{TECHNOLOGY- AND VENDOR-AgNOSTIC}

Due to the many different tools, services, and infrastructures available in the general area of Big Data, an interface ought to be as vendor-independent as possible, while, at the same time, be able to leverage best practices. Hence, a methodology is needed that allows extension of interfaces to adapt and leverage existing approaches, but also allows the interfaces to provide merit in easy specifications that assist the formulation and definition of the NBDRA.

\subsubsection{SUPPORT OF PLUG-IN COMPUTE INFRASTRUCTURE}

As Big Data is not just about hosting data, but about analyzing data, the interfaces provided herein must encapsulate a rich infrastructure environment that is used by data scientists. This includes the ability to integrate (or plug-in) various compute resources and services to provide the necessary compute power to analyze the data. These resources and services include the following:

- Access to hierarchy of compute resources from the laptop/desktop, servers, data clusters, and clouds; 
NIST Big DATA INTEROPERABILITy FRAMEWORK: VoLUME 8, REFERENCE ARCHITECTURE INTERFACES

- The ability to integrate special-purpose hardware such as graphics processing units (GPUs) and field-programmable gate arrays (FPGAs) that are used in accelerated analysis of data; and

- The integration of services including microservices that allow the analysis of the data by delegating them to hosted or dynamically deployed services on the infrastructure of choice.

\subsubsection{ORCHESTRATION OF INFRASTRUCTURE AND SERVICES}

From review of the use case collection, presented in the NBDIF: Volume 3, Use Cases and General Requirements document [4], the need arose to address the mechanism of preparing suitable infrastructures for various use cases. As not every infrastructure is suited for every use case, a custom infrastructure may be needed. As such, this document is not attempting to deliver a single deployed NBDRA, but allow the setup of an infrastructure that satisfies the particular use case. To achieve this task, it is necessary to provision software stacks and services while orchestrating their deployment and leveraging infrastructures. It is not the focus of this document to replace existing orchestration software and services, but provide an interface to them to leverage them as part of defining and creating the infrastructure. Various orchestration frameworks and services could therefore be leveraged, even as part of the same framework, and work in orchestrated fashion to achieve the goal of preparing an infrastructure suitable for one or more applications.

\subsubsection{OrChEstration of BIg Data APPLICATIONS AND EXPERIMENTS}

The creation of the infrastructure suitable for Big Data applications provides the basic computing environment. However, Big Data applications may require the creation of sophisticated applications as part of interactive experiments to analyze and probe the data. For this purpose, the applications must be able to orchestrate and interact with experiments conducted on the data while assuring reproducibility and correctness of the data. For this purpose, a System Orchestrator (either the data scientists or a service acting on behalf of the data scientist) is used as the command center to interact on behalf of the Big Data Application Provider to orchestrate dataflow from Data Provider, carry out the Big Data application life cycle with the help of the Big Data Framework Provider, and enable the Data Consumer to consume Big Data processing results. An interface is needed to describe these interactions and to allow leveraging of experiment management frameworks in scripted fashion. A customization of parameters is needed on several levels. On the highest level, application-motivated parameters are needed to drive the orchestration of the experiment. On lower levels, these high-level parameters may drive and create service-level agreements, augmented specifications, and parameters that could even lead to the orchestration of infrastructure and services to satisfy experiment needs.

\subsubsection{REUSABILITY}

The interfaces provided must encourage reusability of the infrastructure, services, and experiments described by them. This includes (1) reusability of available analytics packages and services for adoption; (2) deployment of customizable analytics tools and services; and (3) operational adjustments that allow the services and infrastructure to be adapted while at the same time allowing for reproducible experiment execution.

\subsubsection{EXECUTION WORKLOADS}

One of the important aspects of distributed Big Data services can be that the data served is simply too big to be moved to a different location. Instead, an interface could allow the description and packaging of analytics algorithms, and potentially also tools, as a payload to a data service. This can be best achieved, not by sending the detailed execution, but by sending an interface description that describes how such an algorithm or tool can be created on the server and be executed under security considerations (integrated with authentication and authorization in mind). 


\subsubsection{SECURITY AND PRIVACY FABRIC REQUIREMENTS}

Although the focus of this document is not security and privacy, which are documented in the NBDIF: Volume 4, Security and Privacy [5], the interfaces defined herein must be capable of integration into a secure reference architecture that supports secure execution, secure data transfer, and privacy. Consequently, the interfaces defined herein can be augmented with frameworks and solutions that provide such mechanisms. Thus, diverse requirement needs stemming from different use cases addressing security need to be distinguished. To contrast that the security requirements between applications can vary drastically, the following example is provided. Although many of the interfaces and their objects to support Big Data applications in physics are similar to those in healthcare, they differ in the integration of security interfaces and policies. While in physics the protection of data is less of an issue, it is a stringent requirement in healthcare. Thus, deriving architectural frameworks for both may use largely similar components, but addressing security issues will be very different. The security of interfaces may be addressed in other documents. In this document, they are considered an advanced use case showcasing that the validity of the specifications introduced here is preserved, even if security and privacy requirements differ vastly among application use cases.

\subsection{COMPONENT-SPECIFIC INTERFACE REQUIREMENTS}

This section summarizes the requirements for the interfaces of the NBDRA components. The five components are listed in Figure 2 and addressed in Section 2.2.1 (System Orchestrator Interface Requirements) through Section 2.2.6 (Big Data Application Provider to Big Data Framework Provider Interface) of this document. The five main functional components of the NBDRA represent the different technical roles within a Big Data system and are the following:

- System Orchestrator: Defines and integrates the required data application activities into an operational vertical system (see Section 2.2.1);

- Data Provider: Introduces new data or information feeds into the Big Data system (see Section 2.2.2);

- Data Consumer: Includes end users or other systems that use the results of the Big Data Application Provider (see Section 2.2.3);

- Big Data Application Provider: Executes a data life cycle to meet security and privacy requirements as well as System Orchestrator-defined requirements (see Section 2.2.4);

- Big Data Framework Provider: Establishes a computing framework in which to execute certain transformation applications while protecting the privacy and integrity of data (see Section 2.2.5); and

- Big Data Application Provider to Framework Provider Interface: Defines an interface between the application specification and the provider (see Section 2.2.6).

\subsubsection{SYSTEM ORCHESTRATOR INTERFACE REQUIREMENTS}

The System Orchestrator role includes defining and integrating the required data application activities into an operational vertical system. Typically, the System Orchestrator involves a collection of more specific roles, performed by one or more actors, which manage and orchestrate the operation of the Big Data system. These actors may be human components, software components, or some combination of the two. The function of the System Orchestrator is to configure and manage the other components of the Big Data architecture to implement one or more workloads that the architecture is designed to execute. The workloads managed by the System Orchestrator may be assigning/provisioning framework components to individual physical or virtual nodes at the lower level, or providing a graphical user interface that supports the specification of workflows linking together multiple applications and components at the higher level. The System Orchestrator may also, through the Management Fabric, monitor the workloads and system to 
confirm that specific quality of service requirements is met for each workload, and may elastically assign and provision additional physical or virtual resources to meet workload requirements resulting from changes/surges in the data or number of users/transactions. The interface to the System Orchestrator must be capable of specifying the task of orchestration the deployment, configuration, and the execution of applications within the NBDRA. A simple vendor-neutral specification to coordinate the various parts either as simple parallel language tasks or as a workflow specification is needed to facilitate the overall coordination. Integration of existing tools and services into the System Orchestrator as extensible interfaces is desirable.

\subsubsection{DATA PROVIDER INTERFACE REQUIREMENTS}

The Data Provider role introduces new data or information feeds into the Big Data system for discovery, access, and transformation by the Big Data system. New data feeds are distinct from the data already in use by the system and residing in the various system repositories. Similar technologies can be used to access both new data feeds and existing data. The Data Provider actors can be anything from a sensor, to a human inputting data manually, to another Big Data system. Interfaces for data providers must be able to specify a data provider so it can be located by a data consumer. It also must include enough details to identify the services offered so they can be pragmatically reused by consumers. Interfaces to describe pipes and filters must be addressed.

\subsubsection{DATA CONSUMER INTERFACE REQUIREMENTS}

Like the Data Provider, the role of Data Consumer within the NBDRA can be an actual end user or another system. In many ways, this role is the mirror image of the Data Provider, with the entire Big Data framework appearing like a Data Provider to the Data Consumer. The activities associated with the Data Consumer role include the following:

- Search and Retrieve,

- Download,

- Analyze Locally,

- Reporting,

- Visualization, and

- Data to Use for Their Own Processes.

The interface for the data consumer must be able to describe the consuming services and how they retrieve information or leverage data consumers.

\subsubsection{Big Data ApPlication Provider INTERFACE ReQUiReMentS}

The Big Data Application Provider role executes a specific set of operations along the data life cycle to meet the requirements established by the System Orchestrator, as well as meeting security and privacy requirements. The Big Data Application Provider is the architecture component that encapsulates the business logic and functionality to be executed by the architecture. The interfaces to describe Big Data applications include interfaces for the various subcomponents including collections, preparation/curation, analytics, visualization, and access. Some of the interfaces used in these subcomponents can be reused from other interfaces, which are introduced in other sections of this document. Where appropriate, application-specific interfaces will be identified and examples provided with a focus on use cases as identified in the NBDIF: Volume 3, Use Cases and General Requirements.

\subsubsection{Collection}

In general, the collection activity of the Big Data Application Provider handles the interface with the Data Provider. This may be a general service, such as a file server or web server configured by the System Orchestrator to accept or perform specific collections of data, or it may be an application-specific service 
NIST Big DATA INTEROPERABILITY FRAMEWORK: VOLUME 8, REFERENCE ARCHITECTURE INTERFACES

designed to pull data or receive pushes of data from the Data Provider. Since this activity is receiving data at a minimum, it must store/buffer the received data until it is persisted through the Big Data Framework Provider. This persistence need not be to physical media but may simply be to an in-memory queue or other service provided by the processing frameworks of the Big Data Framework Provider. The collection activity is likely where the extraction portion of the Extract, Transform, Load (ETL)/Extract, Load, Transform (ELT) cycle is performed. At the initial collection stage, sets of data (e.g., data records) of similar structure are collected (and combined), resulting in uniform security, policy, and other considerations. Initial metadata is created (e.g., subjects with keys are identified) to facilitate subsequent aggregation or look-up methods.

\subsubsection{Preparation}

The preparation activity is where the transformation portion of the ETL/ELT cycle is likely performed, although analytics activity will also likely perform advanced parts of the transformation. Tasks performed by this activity could include data validation (e.g., checksums/hashes, format checks), cleaning (e.g., eliminating bad records/fields), outlier removal, standardization, reformatting, or encapsulating. This activity is also where source data will frequently be persisted to archive storage in the Big Data Framework Provider and provenance data will be verified or attached/associated. Verification or attachment may include optimization of data through manipulations (e.g., deduplication) and indexing to optimize the analytics process. This activity may also aggregate data from different Data Providers, leveraging metadata keys to create an expanded and enhanced data set.

\subsubsection{Analytics}

The analytics activity of the Big Data Application Provider includes the encoding of the low-level business logic of the Big Data system (with higher-level business process logic being encoded by the System Orchestrator). The activity implements the techniques to extract knowledge from the data based on the requirements of the vertical application. The requirements specify the data processing algorithms to produce new insights that will address the technical goal. The analytics activity will leverage the processing frameworks to implement the associated logic. This typically involves the activity providing software that implements the analytic logic to the batch and/or streaming elements of the processing framework for execution. The messaging/communication framework of the Big Data Framework Provider may be used to pass data or control functions to the application logic running in the processing frameworks. The analytic logic may be broken up into multiple modules to be executed by the processing frameworks which communicate, through the messaging/communication framework, with each other and other functions instantiated by the Big Data Application Provider.

\subsubsection{Visualization}

The visualization activity of the Big Data Application Provider prepares elements of the processed data and the output of the analytic activity for presentation to the Data Consumer. The objective of this activity is to format and present data in such a way as to optimally communicate meaning and knowledge. The visualization preparation may involve producing a text-based report or rendering the analytic results as some form of graphic. The resulting output may be a static visualization and may simply be stored through the Big Data Framework Provider for later access. However, the visualization activity frequently interacts with the access activity, the analytics activity, and the Big Data Framework Provider (processing and platform) to provide interactive visualization of the data to the Data Consumer based on parameters provided to the access activity by the Data Consumer. The visualization activity may be completely application-implemented, leverage one or more application libraries, or may use specialized visualization processing frameworks within the Big Data Framework Provider. 


\subsubsection{Access}

The access activity within the Big Data Application Provider is focused on the communication/interaction with the Data Consumer. Like the collection activity, the access activity may be a generic service such as a web server or application server that is configured by the System Orchestrator to handle specific requests from the Data Consumer. This activity would interface with the visualization and analytic activities to respond to requests from the Data Consumer (who may be a person) and uses the processing and platform frameworks to retrieve data to respond to Data Consumer requests. In addition, the access activity confirms that descriptive and administrative metadata and metadata schemes are captured and maintained for access by the Data Consumer and as data is transferred to the Data Consumer. The interface with the Data Consumer may be synchronous or asynchronous in nature and may use a pull or push paradigm for data transfer.

\subsubsection{Big DATA FrameWORK PROVIDER INTERFACE REQUIREMENTS}

Data for Big Data applications are delivered through data providers. They can be either local providers, data contributed by a user, or distributed data providers, data on the Internet. This interface must be able to provide the following functionality:

- Interfaces to files,

- Interfaces to virtual data directories,

- Interfaces to data streams, and

- Interfaces to data filters.

\subsubsection{Infrastructures Interface Requirements}

This Big Data Framework Provider element provides all the resources necessary to host/run the activities of the other components of the Big Data system. Typically, these resources consist of some combination of physical resources, which may host/support similar virtual resources. The NBDRA needs interfaces that can be used to deal with the underlying infrastructure to address networking, computing, and storage.

\subsubsection{Platforms Interface Requirements}

As part of the NBDRA platforms, interfaces are needed that can address platform needs and services for data organization, data distribution, indexed storage, and file systems.

\subsubsection{Processing Interface Requirements}

The processing frameworks for Big Data provide the necessary infrastructure software to support implementation of applications that can deal with the volume, velocity, variety, and variability of data. Processing frameworks define how the computation and processing of the data is organized. Big Data applications rely on various platforms and technologies to meet the challenges of scalable data analytics and operation. A requirement is the ability to interface easily with computing services that offer specific analytics services, batch processing capabilities, interactive analysis, and data streaming.

\subsubsection{Crosscutting Interface Requirements}

Several crosscutting interface requirements within the Big Data Framework Provider include messaging, communication, and resource management. Often these services may be hidden from explicit interface use as they are part of larger systems that expose higher-level functionality through their interfaces. However, such interfaces may also be exposed on a lower level in case finer-grained control is needed. The need for such crosscutting interface requirements will be extracted from the NBDIF: Volume 3, Use Cases and General Requirements document. 


\subsubsection{Messaging/Communications Frameworks}

Messaging and communications frameworks have their roots in the High Performance Computing environments long popular in the scientific and research communities. Messaging/Communications Frameworks were developed to provide application programming interfaces (APIs) for the reliable queuing, transmission, and receipt of data.

\subsubsection{Resource Management Framework}

As Big Data systems have evolved and become more complex, and as businesses work to leverage limited computation and storage resources to address a broader range of applications and business challenges, the requirement to effectively manage those resources has grown significantly. While tools for resource management and elastic computing have expanded and matured in response to the needs of cloud providers and virtualization technologies, Big Data introduces unique requirements for these tools. However, Big Data frameworks tend to fall more into a distributed computing paradigm, which presents additional challenges.

\subsubsection{Big Data Application Provider to Big Data Framework Provider INTERFACE}

The Big Data Framework Provider typically consists of one or more hierarchically organized instances of the components in the NBDRA IT value chain (Figure 2). There is no requirement that all instances at a given level in the hierarchy be of the same technology. In fact, most Big Data implementations are hybrids that combine multiple technology approaches to provide flexibility or meet the complete range of requirements, which are driven from the Big Data Application Provider. 


\section{SPECIFICATION PARADIGM}

This section summarizes the elementary specification paradigm.

\section{4}

525

526

527

528

529

530

531

532

533

534

535

536

537

538

539

540

541

542

543

544

545

546

547

548

549

550

551

\subsection{HYBRID AND MULTIPLE FRAMEWORKS}

To avoid vendor lock-in, Big Data systems must be able to deal with hybrid and multiple frameworks. This is not only true for Clouds, containers, and DevOps, but also for components of the NBDRA.

\subsection{DESIGN BY RESOURCE-ORIENTED ARCHITECTURE}

A resource-oriented architecture represents a software architecture and programming paradigm for designing and developing software in the form of resources. It is often associated with REpresentational State Transfer (REST) interfaces. The resources are software components which can be reused in concrete reference implementations. The service specification is conducted with OpenAPI, allowing use to provide it in a very general form that is independent of the framework or computer language in which the services can be specified. Note that OpenAPI defines services in REST The previous version only specified the resource objects.

\subsection{INTERFACE COMPLIANCY}

Due to the easy extensibility of the resource objects specified in this document and their interfaces, it is important to introduce a terminology that allows the definition of interface compliancy. Three levels of interface compliance are defined as follows:

- Full Compliance: These are reference implementations that provide full compliance to the objects defined in this document. A version number is added to assure that the snapshot in time of the objects is associated with the version. A full compliant framework implements all objects.

- Partial Compliance: These are reference implementations that provide partial compliance to the objects defined in this document. A version number is added to assure that the snapshot in time of the objects is associated with the version. This reference implementation implements a partial list of the objects and interfaces. A document is added that specifies the differences to a full compliant implementation.

- Extended Compliance: In addition to full and partial compliance, additional resources can be identified while documenting additional resource objects and interfaces that are not included in the current specification. The extended compliance document can lead to additional improvements of the current specification. 
553 The specifications in this section are provided through an automated document creation process using the 554 actual OpenAPI specifications yaml files as the source. It is a demonstration that showcases the generation of a fully functioning REST service based on the specifications provided in this document. However, it is expected that scalability, distribution of services, and other advanced options need to be addressed based on application requirements. Limitations of the current implementation are provided in 558 this section.

\subsection{LIST OF SPECIFICATIONS}

560 Table 1 shows the list of specifications included in this version of the document.

Table 1: Specifications

\begin{tabular}{|c|c|c|c|}
\hline Service & Version & Date & Section \\
\hline Alias & 3.2 .0 & 17-06-2019 & Section 4.6.1 \\
\hline Account & 3.2 .0 & 17-06-2019 & Section 4.5.3 \\
\hline Containers & 3.2 .0 & 17-06-2019 & Section 4.10 .1 \\
\hline Database & 3.2 .0 & 17-06-2019 & Section 4.7.3 \\
\hline Default & 3.2 .0 & 17-06-2019 & Section 4.6 .3 \\
\hline Deployment & 3.2 .0 & 17-06-2019 & Section 4.15 .1 \\
\hline Filestore & 3.2 .0 & 17-06-2019 & Section 4.7.1 \\
\hline Filter & 3.2 .0 & 17-06-2019 & Section 4.14 .2 \\
\hline Flavor & 3.2 .0 & 17-06-2019 & Section 4.9.2 \\
\hline Image & 3.2 .0 & 17-06-2019 & Section 4.9.1 \\
\hline MapReduce & 3.2 .0 & 17-06-2019 & Section 4.11 .1 \\
\hline Microservice & 3.2 .0 & $17-06-2019$ & Section 4.12 .1 \\
\hline Nic & 3.2 .0 & 17-06-2019 & Section 4.9.5 \\
\hline Network of Nodes & 3.2 .0 & 17-06-2019 & Section 4.8 .2 \\
\hline Organization & 3.2 .0 & 17-06-2019 & Section 4.5.1 \\
\hline Public Key Store & 3.2 .0 & 17-06-2019 & Section 4.5 .4 \\
\hline Queue & 3.2 .0 & 17-06-2019 & Section 4.8.4 \\
\hline Replica & 3.2 .0 & 17-06-2019 & Section 4.7 .2 \\
\hline Reservation & 3.2 .0 & 17-06-2019 & Section 4.13 .1 \\
\hline Scheduler & 3.2 .0 & 17-06-2019 & Section 4.8.3 \\
\hline
\end{tabular}


NIST Big DATA INTEROPERABILITY FRAMEWORK: VOLUME 8, REFERENCE ARCHITECTURE INTERFACES

\begin{tabular}{llll} 
Service & Version & Date & Section \\
\hline Secgroup & 3.2 .0 & $17-06-2019$ & Section 4.9.4 \\
\hline Stream & 3.2 .0 & $17-06-2019$ & Section 4.14.1 \\
\hline Timestamp & 3.2 .0 & $17-06-2019$ & Section 4.4.1 \\
\hline User & 3.2 .0 & $17-06-2019$ & Section 4.5.2 \\
\hline Variables & 3.2 .0 & $17-06-2019$ & Section 4.6.2 \\
\hline Virtual Cluster & 3.2 .0 & $17-06-2019$ & Section 4.8.1 \\
\hline Virtual Directory & 3.2 .0 & $17-06-2019$ & Section 4.7.4 \\
\hline Virtual Machine & 3.2 .0 & $17-06-2019$ & Section 4.9.3
\end{tabular}

562 Figure 3 shows the provider view of the specification resources.

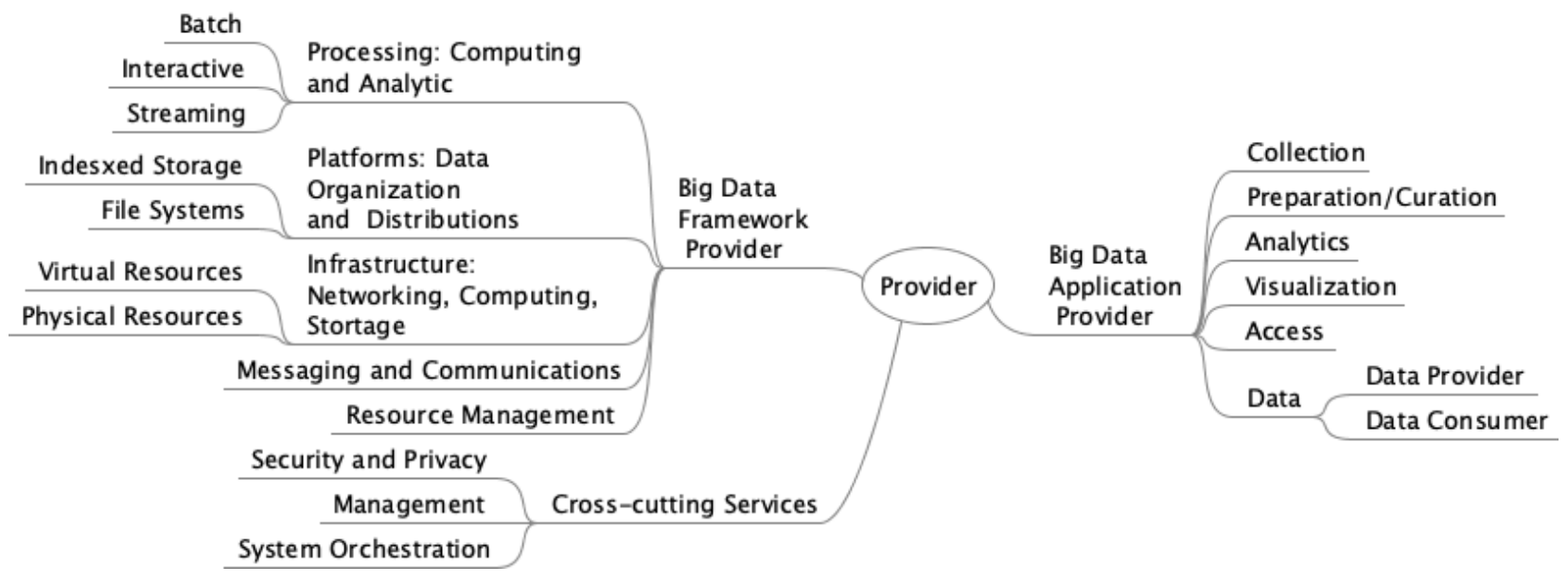

Figure 3: Provider View 
Figure 4 shows the resources view of the specification resources.

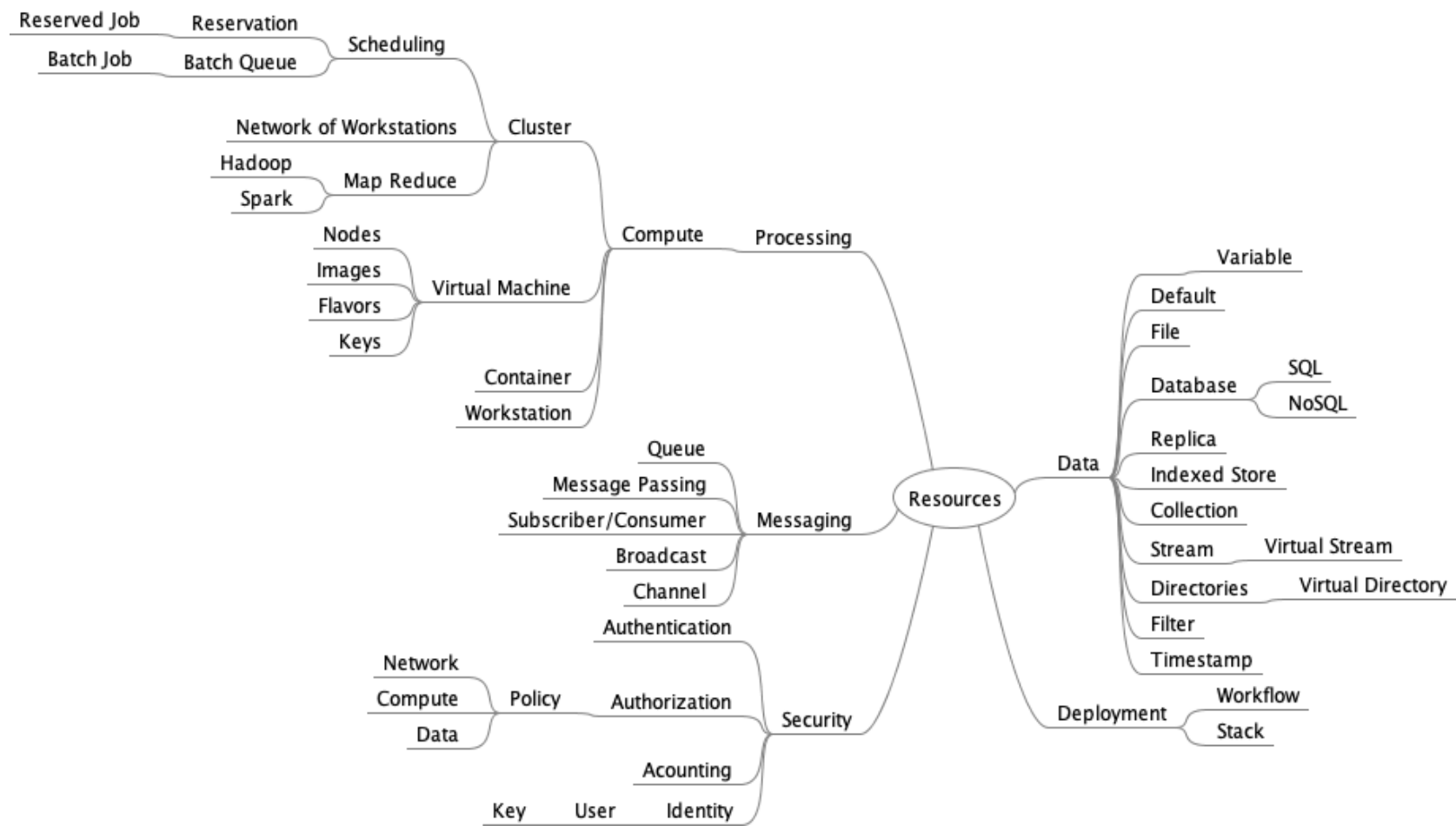

Figure 4: Resource View

\subsection{AUTHENTICATION}

Mechanisms are not included in this specification to manage authentication to external services. However, the working group has shown multiple solutions to this as part of Cloudmesh. These include the possibility of the following:

- Local configuration file: A configuration file is managed locally to allow access to the clouds. It is the designer's responsibility not to expose such credentials.

- Session based authentication: No passwords are stored in the configuration file and access is granted on a per session basis where the password needs to be entered.

- Service based authentication: The authentication is delegated to an external process. The service that acts on behalf of the user needs to have access to the appropriate cloud provider credentials.

\subsection{STATUS CODES AND ERROR RESPONSES}

In case of an error or a successful response, the response header contains a HyperText Transfer Protocol (HTTP) code [19]. The response body usually contains the following:

- $\quad$ The HTTP response code;

- An accompanying message for the HTTP response code; and

- A field or object where the error occurred. 
Table 2: HTTP Response Codes

\begin{tabular}{lll} 
HTTP Response & Operation & Description \\
\hline $\mathbf{2 0 0}$ Ok & GET, PUT, DELETE & No error, operation successful. \\
\hline $\mathbf{2 0 1}$ Created & POST & Successful creation of a resource. \\
\hline $\mathbf{2 0 4}$ No Content & GET, PUT, DELETE & Successful but no content. \\
\hline $\mathbf{4 0 0}$ Bad Request & GET, POST, PUT, & The request could not be understood. \\
& DELETE & \\
\hline $\mathbf{4 0 1}$ & GET, POST, PUT, & User must authorize. \\
\hline Unauthorized & DELETE & \\
\hline $\mathbf{4 0 3}$ Forbidden & GET, POST, PUT, & $\begin{array}{l}\text { The request has been refused due to authorization } \\
\text { failure. }\end{array}$ \\
\hline $\mathbf{4 0 4}$ Not Found & GELETE & The requested resource could not be found. \\
& DELETE & \\
\hline $\mathbf{4 0 5}$ Not Allowed & GET, POST, PUT, & The method is not allowed on the resource. \\
& DELETE & \\
\hline $\mathbf{5 0 0}$ Server Error & GET, POST PUT & Internal Server error.
\end{tabular}

583 In the specification such responses are indicated and if a simple response is returned the term Message is 584 used.

\subsection{TIMESTAMP}

Timestamps can be used in conjunction with any server side implementation of the interfaces. It can be useful to return information about when a particular resource has been created, updated, or accessed. To simplify the specification in the document, a timestamp is not explicitly listed as part of the resource, but it can be assumed that it may be added as part of the service implementation. To obtain an example timestamp a simple get function is provided.

\subsubsection{TIMESTAMP}

Data often needs to be time stamped to indicate when it has been accessed, created, or modified. All objects defined in this document will have, in their final version, a timestamp. The date-time string is defined in RFC3339 [20].

\subsubsection{Schema Timestamp}

\begin{tabular}{lll} 
Property & Type & Description \\
\hline accessed & string(date-time) & The time stamp when the object was last accessed \\
created & string(date-time) & The time stamp when the object was created \\
modified & string(date-time) & The time stamp when the object was modified
\end{tabular}


NIST Big DATA INTEROPERABILITY FRAMEWORK: Volume 8, REFERENCE ARCHITECTURE INTERFACES

596

4.4.1.2 Paths

\begin{tabular}{lll} 
HTTP & Path & Summary \\
\hline get & /timestamp & Returns the timestamp
\end{tabular}

\subsection{Itimestamp}

\subsection{GET/timestamp}

599 Returns the timestamp

600 Responses

Code Description Schema

200 The current time and date string

601

602

603

604

605

606

607

608

609

610

611

612

613

614

615

616

617

618

619

620

621

622

623

624

625

626

627

628

629

630

631

632

633

634

635

636

637

638

639

640

641

642

643

644

645

646

647

\subsubsection{3 timestamp.yaml}

openapi: "3.0.2"

info:

version: 3.2 .0

$x$-date: 17-06-2019

$x$-status: defined

title: Timestamp

description: |-

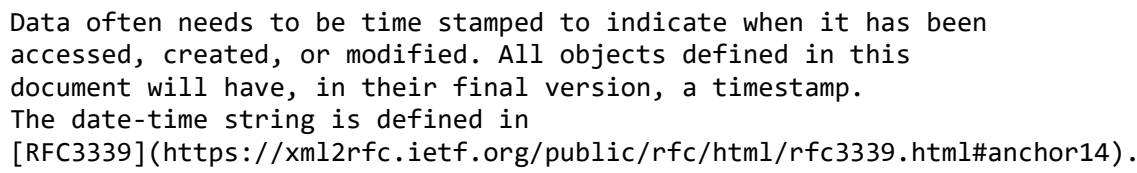




\subsection{IDENTITY}

As part of services an identity often needs to be specified. Such persons [21] are often part of groups.

Three important terms related to the identity are distinguished as follows:

- Organization: The information representing an Organization that manages a Big Data Service (Section 4.5.1)

- Group: A group that a person may belong to that is important to define access to services (included in Section 4.5.1)

- User: The information identifying the profile of a person (Section 4.5.2)

\subsubsection{ORGANIZATION}

An important concept in many services is the management of a group of users in an organization. Within an organization, different groups of users are distinguished. Groups can be used to characterize roles that users can fulfill. Users can belong to multiple groups. Such groups can also be used to specify access rights to services.

\subsubsection{Schema Organization}

\begin{tabular}{lll} 
Property & Type & Description \\
\hline name & string & Name of the organization \\
users & array[User] & List of users
\end{tabular}

\subsubsection{Schema Group}

\begin{tabular}{lll} 
Property & Type & Description \\
\hline name & string & The name of the group \\
description & string & The description of the group \\
users & array[string] & The user names that are members of the group
\end{tabular}

\subsubsection{Paths}

\begin{tabular}{ll} 
HTTP & Path \\
\hline get & /organization \\
get & /organization/ $\{$ name $\}$ \\
put & /organization/ $\{$ name $\}$ \\
& \\
delete & /organization/ $\{$ name $\}$
\end{tabular}

Summary

Returns a list of organizations

Returns the named organization

Uploads an organization to the list of organizations

Deletes the named organization 


\begin{tabular}{|c|c|c|}
\hline HTTP & Path & Summary \\
\hline get & /organization/\{name\}/user & Returns all users of the organization \\
\hline get & /organization/ $\{$ name $\} /$ user/ $\{$ user $\}$ & Returns the specific user of that organization \\
\hline put & /organization/ $\{$ name $\} /$ user/ $\{$ user $\}$ & Updates or adds a user in the organization \\
\hline delete & /organization/ $\{$ name $\} /$ user/ $\{$ user\} & Delete an user in the organization \\
\hline get & /organization/\{name\}/group/ & Returns all group names \\
\hline get & /organization/\{name\}/group/\{group\} & Returns the specific group of that organization \\
\hline put & /organization/\{name\}/group/\{group\} & Updates or adds a group in the organization \\
\hline delete & /organization/\{name\}/group/\{group\} & Delete a group in the organization \\
\hline put & /organization/\{name\}/group/\{group $\} /\{$ user $\}$ & Updates or adds a user name to the group \\
\hline delete & /organization/\{name\}/group/\{group $\} /\{$ user $\}$ & Delete a user in the group \\
\hline
\end{tabular}

\section{4.5.1.3.1 lorganization}

676 4.5.1.3.1.1 GET /organization

677 Returns a list of all organizations

678 Responses

Code Description Schema

200 The list of organizations array[Organization]

$401 \quad$ Not authorized String

679 4.5.1.3.2 lorganization/\{name\}

680 4.5.1.3.2.1 GET/organization/\{name\}

681 Returns an organization by name

682 Responses

Code Description Schema

200 Returning the information of the organization Organization

401 Not authorized String

404 The named organization could not be found String

683 Parameters

\begin{tabular}{lllll} 
Name & Located in & Description & Required & Schema \\
\hline name & path & The name of the organization & True & String
\end{tabular}

685 Uploads an organization to the list of organizations 
Responses

\begin{tabular}{lll} 
Code & Description & Schema \\
\hline 200 & Organization created or updated & String \\
401 & Not authorized & String \\
404 & The organization could not be found & String
\end{tabular}

687 Request Body

\begin{tabular}{llll} 
Located in & Description & Required & Schema \\
\hline Body & The organization to be uploaded & True & Organization
\end{tabular}

\subsection{DELETE /organization/\{name\}}

689 Deletes an organization by name

690 Responses

Code Description Schema

200 Deletion successful String

401 Not authorized String

404 The named organization could not be found String

691

Parameters

\begin{tabular}{lllll} 
Name & Located in & Description & Required & Schema \\
\hline name & path & The name of the organization & True & String
\end{tabular}

692 4.5.1.3.3 lorganization/\{name\}/user

693 4.5.1.3.3.1 GET/organization/\{name\}/user

694 Returns all users of the organization

695 Responses

Code Description Schema

200 The organization Organization

401 Not authorized String

696 Parameters

\begin{tabular}{lllll} 
Name & Located in & Description & Required & Schema \\
\hline name & path & The name of the organization & True & String
\end{tabular}

697 4.5.1.3.4 lorganization/\{name\}/userl\{user\}

698 4.5.1.3.4.1 GET/organization/\{name\}/user/\{user\}

699 Returns the specific user of that organization 
Responses

Code Description

Schema

200 The user

$\underline{\text { User }}$

401 Not authorized

String

404 The organization or user could not be found String

701 Parameters

\begin{tabular}{lllll} 
Name & Located in & Description & Required & Schema \\
\hline name & path & The name of the organization & True & String \\
user & path & The user name & True & String
\end{tabular}

702 4.5.1.3.4.2 PUT /organization/\{name\}/user/\{user\}

703 Updates or adds a user in the organization

704 Responses

\begin{tabular}{lll} 
Code & Description & Schema \\
\hline 200 & User added successfully & String \\
401 & Not authorized & String
\end{tabular}

404 The organization or user could not be found String

705 Parameters

\begin{tabular}{lllll} 
Name & Located in & Description & Required & Schema \\
\hline name & path & The name of the organization & True & String \\
user & path & The user name & True & String
\end{tabular}

706 Request Body

\begin{tabular}{llll} 
Located in & Description & Required & Schema \\
\hline Body & The user to be uploaded & True & $\underline{\text { User }}$
\end{tabular}

\subsection{DELETE /organization/\{name\}/user/\{user\}}

708 Delete a user in the organization

709 Responses

\begin{tabular}{lll} 
Code & Description & Schema \\
\hline 200 & Deletion successful & String \\
401 & Not authorized & String \\
404 & The organization or user could not be found & String
\end{tabular}


NIST Big DATA INTEROPERABILITY FRAMEWORK: VOLUME 8, REFERENCE ARCHITECTURE INTERFACES

710

Parameters

\begin{tabular}{lllll} 
Name & Located in & Description & Required & Schema \\
\hline name & path & The name of the organization & True & String \\
user & path & The user name & True & String
\end{tabular}

711 4.5.1.3.5 lorganization/\{name\}/group/

\section{4.5.1.3.5.1 GET/organization/\{name\}/group/}

713 Returns all group names

714 Responses

Code Description Schema

200 Returning the information of the group array[String]

400 No group found String

401 Not authorized String

404 The organization or group could not be found String

715 Parameters

\begin{tabular}{lllll} 
Name & Located in & Description & Required & Schema \\
\hline name & path & The name of the organization & True & String
\end{tabular}

716 4.5.1.3.6 lorganization/\{name\}/group/\{group\}

717 4.5.1.3.6.1 GET/organization/\{name\}/group/\{group\}

718 Returns the specific group of that organization

719 Responses

Code Description Schema

200 The group $\quad$ Group

401 Not authorized String

404 The organization or group could not be found String

720 Parameters

Name Located in Description Required Schema

name path The name of the organization True String

group path The group name True String

\section{4.5.1.3.6.2 PUT /organization/\{name\}/group/\{group\}}

722 Updates or adds a group in the organization 
Responses

Code Description

Schema

200 Group added successfully

String

401 Not authorized

String

404 The organization or group could not be found String

724 Parameters

\begin{tabular}{lllll} 
Name & Located in & Description & Required & Schema \\
\hline name & path & The name of the group & True & String \\
group & path & The group name & True & String
\end{tabular}

725 4.5.1.3.6.3 DELETE /organization/\{name\}/group/\{group\}

726 Delete a group in the organization

727 Responses

\begin{tabular}{lll} 
Code & Description & Schema \\
\hline 200 & Deletion successful & String \\
401 & Not authorized & String
\end{tabular}

404 The organization or group could not be found String

728 Parameters

\begin{tabular}{lllll} 
Name & Located in & Description & Required & Schema \\
\hline name & path & The name of the organization & True & String \\
group & path & The group name & True & String
\end{tabular}

729 4.5.1.3.7 lorganization/\{name\}/group/\{group\}/\{user\}

730 4.5.1.3.7.1 PUT /organization/\{name\}/group/\{group\}/\{user\}

731 Updates or adds a user name to the group

732 Responses

\begin{tabular}{lll} 
Code & Description & Schema \\
\hline 200 & User added successfully & String \\
401 & Not authorized & String
\end{tabular}

404 The organization, group, or user could not be found String

733 Parameters

\begin{tabular}{lllll} 
Name & Located in & Description & Required & Schema \\
\hline name & path & The name of the group & True & String \\
group & path & The group name & True & String \\
user & path & The user name & True & String
\end{tabular}




\subsection{DELETE /organization/\{name\}/group/\{group\}/\{user\}}

735 Delete a user in the group

736 Responses

Code Description Schema

$\begin{array}{lll}200 & \text { Deletion successful } & \text { String } \\ 401 & \text { Not authorized } & \text { String } \\ 404 & \text { The organization, group, or user could not be found } & \text { String }\end{array}$

Parameters

\begin{tabular}{lllll} 
Name & Located in & Description & Required & Schema \\
\hline name & path & The name of the organization & True & String \\
group & path & The group name & True & String \\
user & path & The user name & True & String
\end{tabular}

\subsubsection{4 organization.yaml}

openapi: "3.0.2"

info:

version: 3.2 .0

$x$-date: $17-06-2019$

$x$-status: defined

title: Organization

description: |-

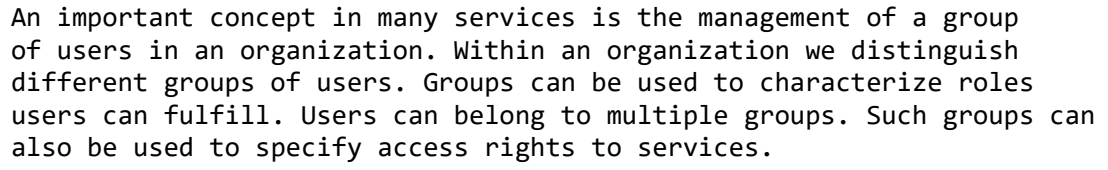




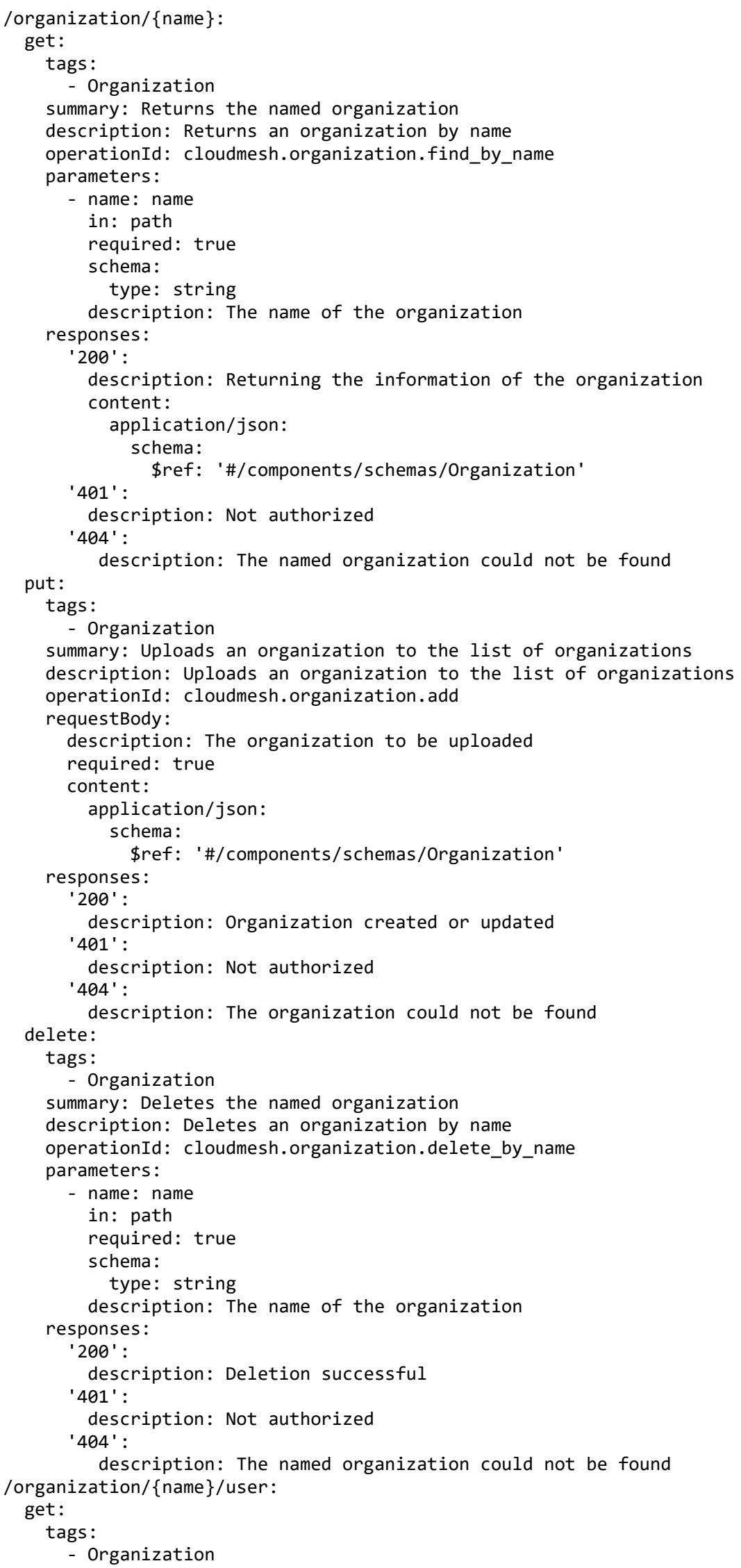


850

851

852

853

854

855

856

857

858

859

860

861

862

863

864

865

866

867

868

869

870

871

872

873

874

875

876

877

878

879

880

881

882

883

884

885

886

887

888

889

890

891

892

893

894

895

896

897

898

899

900

901

902

903

904

905

906

907

908

909

910

911

912

913

914

915

916

917

918

summary: Returns all users of the organization

description: Returns all users of the organization

operationId: cloudmesh.organization.user.list

parameters:

- name: name

in: path

required: true

schema:

type: string

description: The name of the organization responses:

' 200 ' :

description: The organization

content:

application/json:

schema:

\$ref: "\#/components/schemas/Organization"

'401' :

description: Not authorized

/organization/ $\{$ name $\} /$ user $/\{$ user $\}$ :

get:

tags :

- Organization

summary: Returns the specific user of that organization

description: Returns the specific user of that organization

operationId: cloudmesh.organization.user.get_by_name

parameters:

- name: name

in: path

required: true

schema:

type: string

description: The name of the organization

- name: user

description: The user name

in: path

required: true

schema:

type: string

responses:

'200' :

description: The user

content:

application/json:

schema:

' 401 ' :

\$ref: "user.yaml\#/components/schemas/User"

description: Not authorized

' 404 ' : put :

description: The organization or user could not be found

tags:

- Organization

summary: Updates or adds a user in the organization

description: Updates or adds a user in the organization

operationId: cloudmesh.organization.user.add

parameters:

- name: name

in: path

required: true

schema:

type: string

description: The name of the organization

- name: user

description: The user name

in: path

required: true

schema:

type: string 
NIST Big DATA INTEROPERABILITY FRAMEWORK: VOLUME 8, REFERENCE ARCHITECTURE INTERFACES

919

920

921

922

923

924

925

926

927

928

929

930

931

932

933

934

935

936

937

938

939

940

941

942

943

944

945

946

947

948

949

950

951

952

953

954

955

956

957

958

959

960

961

962

963

964

965

966

967

968

969

970

971

972

973

974

975

976

977

978

979

980

981

982

983

984

985

986

987

requestBody:

description: The user to be uploaded

required: true

content:

application/json:

schema:

\$ref: 'user.yaml\#/components/schemas/User'

responses:

'200' :

description: User added successfully

' 401 ' :

description: Not authorized

' 404 ' :

description: The organization or user could not be found $\backslash$ delete:

tags:

- Organization

summary: Delete an user in the organization

description: Delete an user in the organization

operationId: cloudmesh.organization.user.delete

parameters:

- name: name

in: path

required: true

schema:

type: string

description: The name of the organization

- name: user

description: The user name

in: path

required: true

schema:

type: string

responses:

'200' :

description: Deletion successful

' 401 ' :

description: Not authorized

'404' :

description: The organization or user could not be found /organization/\{name\}/group/:

get:

tags:

- Organization

summary: Returns all group names

description: Returns all group names

operationId: cloudmesh.organization.group.list

parameters :

- name: name

in: path

required: true

schema:

type: string

description: The name of the organization responses:

'200' :

description: Returning the information of the group

content:

application/json:

schema:

type: array

items:

'400' :

type: string

description: No group found

' 401 ' :

description: Not authorized

' 404 ' :

description: The organization or group could not be found 


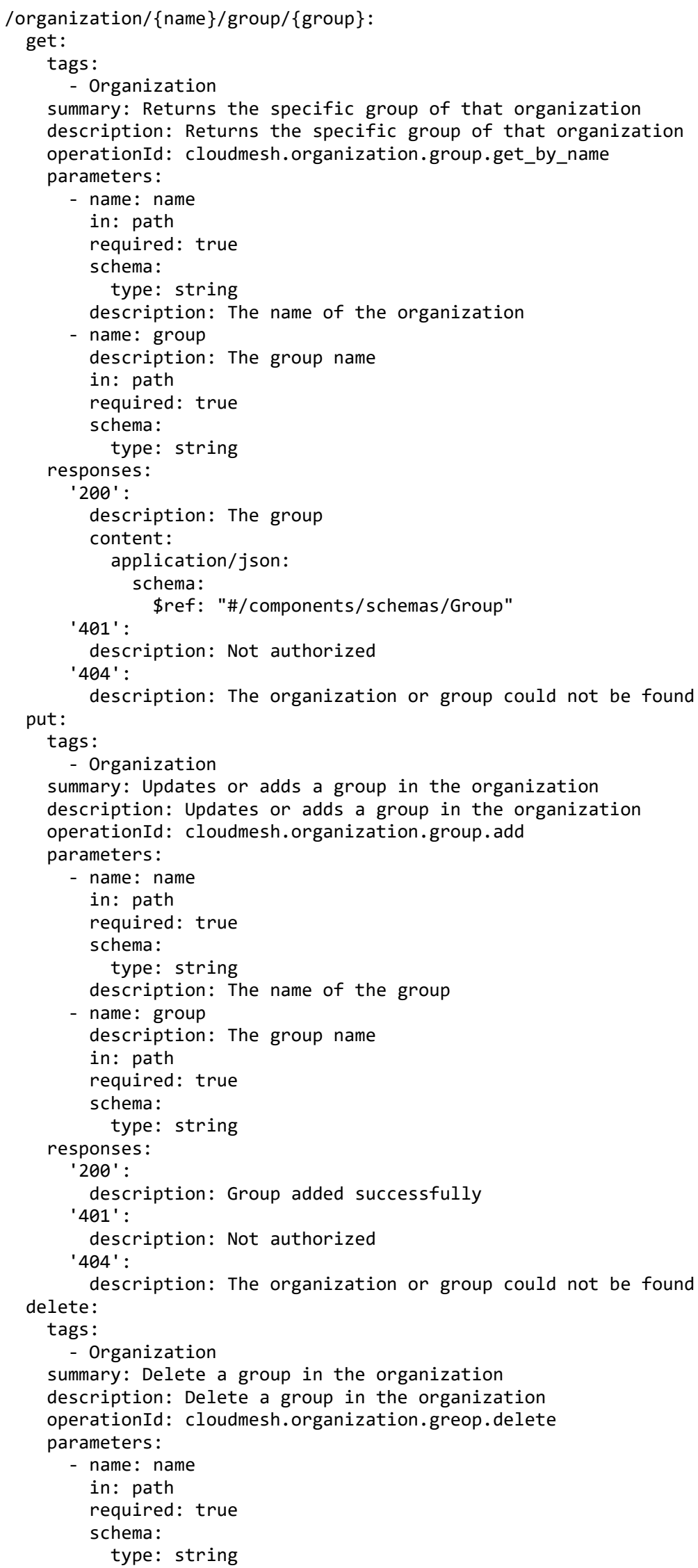


description: The name of the organization

- name: group

description: The group name

in: path

required: true

schema:

type: string

responses:

'200':

description: Deletion successful

' 401 ' :

description: Not authorized

'404' :

description: The organization or group could not be found /organization/\{name\}/group/\{group\}/\{user\}:

put:

tags:

- Organization

summary: Updates or adds a user name to the group

description: Updates or adds a user name to the group

operationId: cloudmesh.organization.group.user.add

parameters:

- name: name

in: path

required: true

schema:

type: string

description: The name of the group

- name: group

description: The group name

in: path

required: true

schema:

type: string

- name: user

description: The user name

in: path

required: true

schema:

type: string

responses:

'200' :

description: User added successfully

' 401 ' :

description: Not authorized

'404':

delete:

description: The organization, group, or user could not be found

tags:

- Organization

summary: Delete a user in the group

description: Delete a user in the group

operationId: cloudmesh.organization.greop.delete.user

parameters:

- name: name

in: path

required: true

schema:

type: string

description: The name of the organization

- name: group

description: The group name

in: path

required: true

schema:

type: string

- name: user

description: The user name in: path 
NIST Big DATA INTEROPERABILITY FRAMEWORK: VOLUME 8, REFERENCE ARCHITECTURE INTERFACES

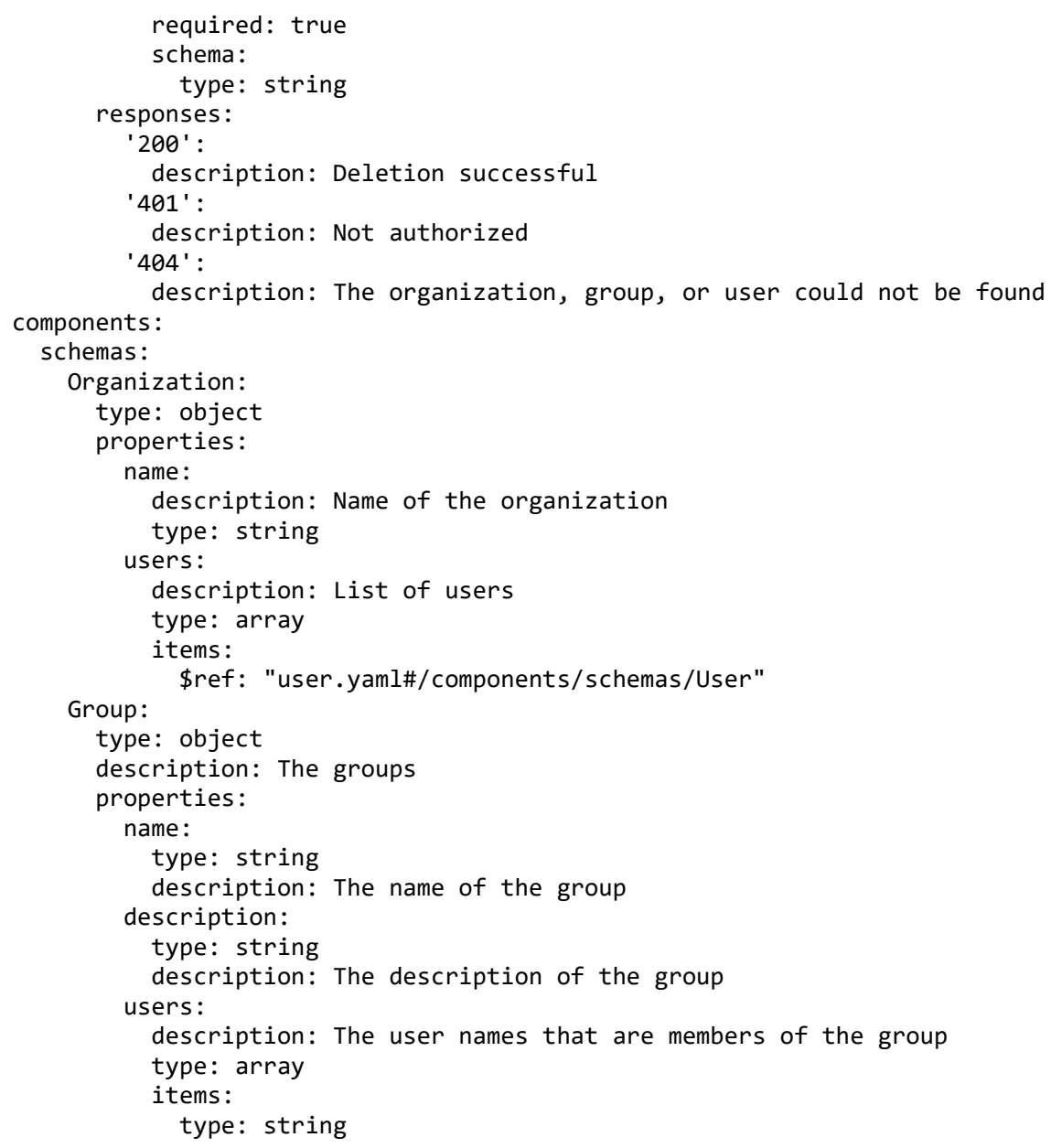

\subsubsection{USER}

Services need to specify which users have access to them. User information can be reused in other services and organized in a virtual organization. A user can be added to a named list of users within this organization. A group associated with the user can be used to augment users to be part of one or more groups.

\subsubsection{Schema User}

\begin{tabular}{lll} 
Property & Type & Description \\
\hline username & String & The unique username associated with the user \\
firstname & String & The firstname of the user \\
lastname & string & The lastname of the user \\
email & string & The email of the user \\
comment & string & A comment regarding the user \\
publickey & string & The public key of the user
\end{tabular}


4.5.2.2 Paths

\begin{tabular}{lll} 
HTTP & Path & Summary \\
\hline get & /user & Returns a list of users \\
get & $/$ user $/\{$ name $\}$ & Returns the named user \\
put & $/$ user $/\{$ name $\}$ & Uploads a user to the list of users \\
delete & $/$ user $/\{$ name $\}$ & Deletes the named user
\end{tabular}

$1172 \quad$ 4.5.2.2.1 /user

1173 4.5.2.2.1.1 GET/user

1174 Returns a list of all users

1175 Responses

\begin{tabular}{lll} 
Code & Description & Schema \\
\hline 200 & The list of users & array[ $\underline{\text { User] }}$
\end{tabular}

401 Not authorized String

1176 4.5.2.2.2 luser/\{name\}

1177 4.5.2.2.2.1 GET/user/\{name\}

1178 Returns a user by name

1179 Responses

Code Description Schema

200 Returning the information of the user $\underline{\text { User }}$

401 Not authorized String

404 The named user could not be found String

1180 Parameters

\begin{tabular}{lllll} 
Name & Located in & Description & Required & Schema \\
\hline name & path & The name of the user & True & String
\end{tabular}

1181 4.5.2.2.2.2 PUT /user/\{name\}

1182 Uploads a user to the list of users

1183 Responses

\begin{tabular}{lll} 
Code & Description & Schema \\
\hline 200 & User updated & String \\
401 & Not authorized & String \\
404 & The named user could not be found & String
\end{tabular}


NIST Big DATA INTEROPERABILITY FRAMEWORK: VOLUME 8, REFERENCE ARCHITECTURE INTERFACES

1184

Request Body

Located in Description Required Schema

Body The user to be uploaded True $\underline{\text { User }}$

1185

\subsection{DELETE /user/\{name\}}

1186

Deletes a user by name

1187

Responses

Code Description

Schema

200 Deletion successful

String

$401 \quad$ Not authorized

String

404 The named user could not be found String

1188

Parameters

Name Located in Description Required Schema

name path The name of the user True String

\subsubsection{3 user.yaml}

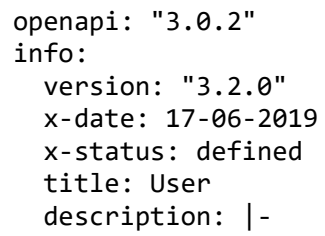


NIST Big DATA INTEROPERABILITY FRAMEWORK: VOLUME 8, REFERENCE ARCHITECTURE INTERFACES

1229

1230

1231

1232

1233

1234

1235

1236

1237

1238

1239

1240

1241

1242

1243

1244

1245

1246

1247

1248

1249

1250

1251

1252

1253

1254

1255

1256

1257

1258

1259

1260

1261

1262

1263

1264

1265

1266

1267

1268

1269

1270

1271

1272

1273

1274

1275

1276

1277

1278

1279

1280

1281

1282

1283

1284

1285

1286

1287

1288

1289

1290

1291

1292

1293

1294

1295

1296

1297

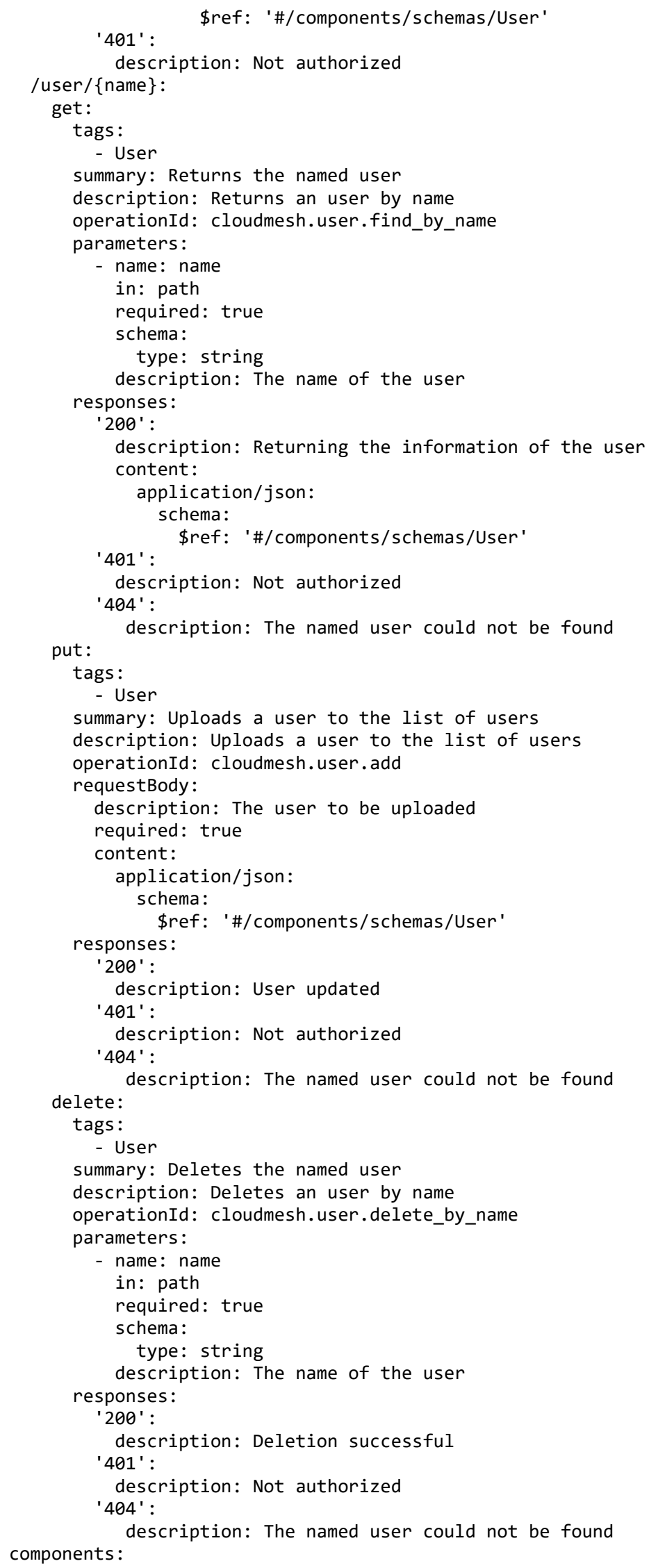


1298

1299

1300

1301

1302

1303

1304

1305

1306

1307

1308

1309

1310

1311

1312

1313

1314

1315

1316

1317

1318

1319

1320

1321

1322

1323

1324

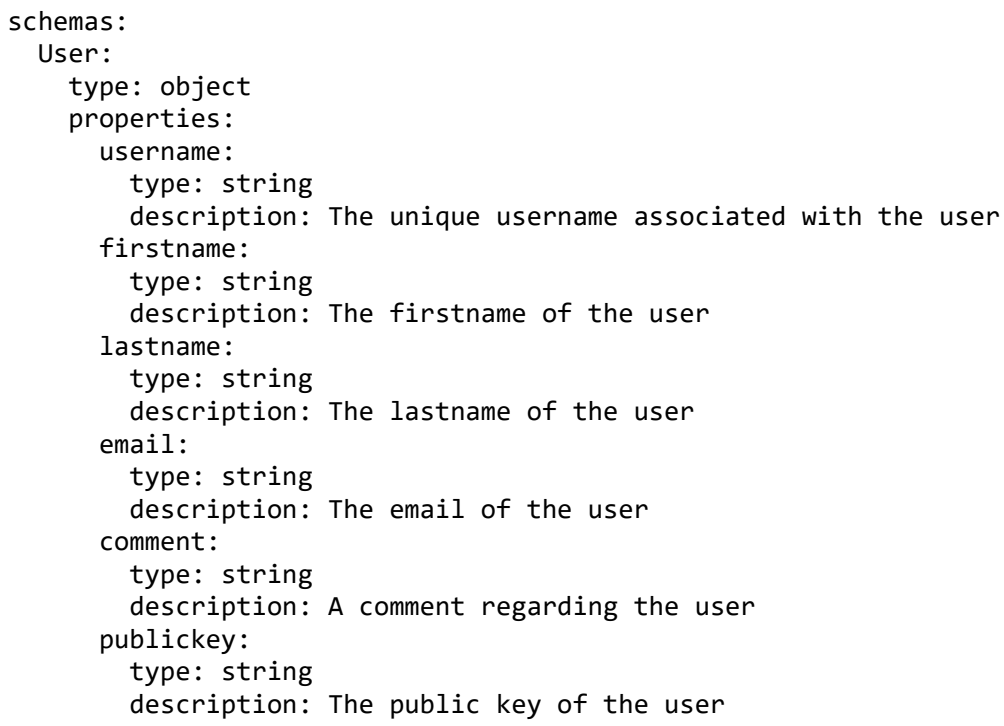

\subsubsection{ACCOUNT}

Accounting can be used to charge the use of resources. Accounting can be implemented on a variety of resources, such as users, groups, or organizations. It is up to the implementer to provide rules and cost for the accounting. If needed, multiple accounting resources can be implemented.

\subsubsection{Schema Account}

Property Type Description

name string name of account

description string the purpose of the account

charge integer The current charge of the account

unit string the unit in which the account is charged and the charge value is stored

1325 4.5.3.2 Paths

\begin{tabular}{lll} 
HTTP & Path & Summary \\
\hline get & /account & Returns the accounts \\
get & $/$ account $/\{$ name $\}$ & Returns the named account \\
put & $/$ account $/\{$ name $\}$ & Set the value of an account \\
delete & $/$ account $/\{$ name $\}$ & Deletes the named account
\end{tabular}

\subsubsection{1 laccount}

\subsection{GET /account}

1328 
NIST Big DATA INTEROPERABILITY FRAMEWORK: VOLUME 8, REFERENCE ARCHITECTURE INTERFACES

1329

Responses

Code Description

Schema

200 The list of accounts array[Account]

$401 \quad$ Not authorized String

$1330 \quad$ 4.5.3.2.2 laccount/\{name $\}$

1331 4.5.3.2.2.1 GET /account/\{name\}

1332 Returns the named account

1333 Responses

Code Description Schema

200 Returning the information of the account $\underline{\text { Account }}$

401 Not authorized String

404 The named account could not be found String

1334 Parameters

Name Located in Description Required Schema

name path The name of the account True String

1335 4.5.3.2.2.2 PUT /account/\{name\}

1336 Set the value of the named account

1337 Responses

Code Description Schema

200 Account updated or created String

400 Error updating account String

401 Not authorized String

1338 Request Body

Located in Description Required Schema

Body The account and its value True $\underline{\text { Account }}$

1339 4.5.3.2.2.3 DELETE /account/\{name\}

1340 Deletes an account by name

1341 Responses

\begin{tabular}{lll} 
Code & Description & Schema \\
\hline 200 & Deletion successful & String \\
401 & Not authorized & String \\
404 & The named account could not be found & String
\end{tabular}


NIST Big DATA INTEROPERABILITY FRAMEWORK: Volume 8, REFERENCE ARCHITECTURE INTERFACES

Parameters

\begin{tabular}{lllll} 
Name & Located in & Description & Required & Schema \\
\hline name & path & The name of the account & True & String
\end{tabular}

1343

1344

1345

건 1346

1347

1348

1349

1350

1351

1352

1353

1354

1355

1356

1357

1358

1359

1360

1361

1362

1363

1364

1365

1366

1367

1368

1369

1370

1371

1372

1373

1374

1375

1376

1377

1378

1379

1380

1381

1382

1383

1384

1385

1386

1387

1388

1389

1390

1391

1392

1393

1394

1395

1396

1397

1398

1399

1400

1401

1402

1403

\subsubsection{3 account.yaml}

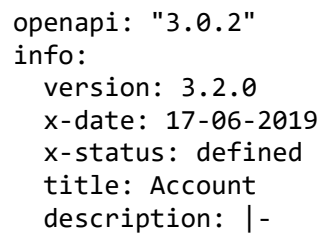


NIST Big DATA INTEROPERABILITY FRAMEWORK: Volume 8, REFERENCE ARCHITECTURE INTERFACES

1404

1405

1406

1407

1408

1409

1410

1411

1412

1413

1414

검 1415

1416

1417

1418

1419

1420

1421

1422

1423

1424

(2) 1425

๘. 1426

1427

1428

1429

1430

1431

1432

1433

1434

1435

1436

1437

1438

1439

1440

1441

1442

- 1443

1444

1445

1446

1447

1448

1449

1450

1451

1452

1453

1454

1455

1456

1457

1458

1459

- 1460

ב 1461

1462

1463

1464

1465

1466

1467

1468

1469

1470

1471

1472

content:

application/json:

schema:

'401' :

\$ref: '\#/components/schemas/Account'

description: Not authorized

' 404 ' :

description: The named account could not be found

put:

tags:

- Account

summary: Set the value of an account

description: Set the value of the named account

operationId: cloudmesh.account.add

requestBody:

description: The account and its value

required: true

content:

application/json:

schema:

responses:

\$ref: '\#/components/schemas/Account'

' 200 ' :

description: Account updated or created

'400' :

description: Error updating account

' 401 ' :

delete:

description: Not authorized

tags:

- Account

summary: Deletes the named account

description: Deletes an account by name

operationId: cloudmesh.account.delete_by_name

parameters:

- name: name

in: path

required: true

schema:

type: string

description: The name of the account

responses:

' 200 ' :

description: Deletion successful

' 401 ' :

description: Not authorized

' 404 ' :

components:

description: The named account could not be found

schemas:

Account:

type: object

description: account information including finances and summary.

properties:

name:

type: string

description: name of account

example: GroupAccount

description:

type: string

description: the purpose of the account

example: "Charging all users for the account access"

charge:

type: integer

minimum: 0

description: The current charge of the account example: 1

unit:

type: string 
1473

1474

1475

1476

1477

1478

description: the unit in which the account is charged and the charge value is stored example: SU

\subsubsection{Public Key Store}

Many services and frameworks use Secure Shell (SSH) keys to authenticate. This service allows the convenient storage of the public keys.

\subsubsection{Schema Key}

\begin{tabular}{lrl} 
Property & Type & Description \\
\hline name & string & The name of the public key \\
value & string & The value of the public key \\
kind & string & $\begin{array}{l}\text { The key kind such as Rivest-Shamir-Adleman (RSA) and Digital Signature } \\
\text { Algorithm (DSA) }\end{array}$ \\
group & string & $\begin{array}{l}\text { An optional group name allowing to group keys to create custom key groups } \\
\text { within the public key store }\end{array}$ \\
comment & string & A comment for the public key \\
uri & string & The Uniform Resource Identifier (URI) of the public key if any \\
fingerprint & string & The fingerprint of the public key
\end{tabular}

\subsubsection{Paths}

\begin{tabular}{lll} 
HTTP & Path & Summary \\
\hline get & /key & Returns a list of keys
\end{tabular}

get $\quad /$ key/\{name\} Returns the named key

put $/$ key/\{name $\quad$ Set a key

delete $/$ key/\{name\} Deletes the named key

\subsubsection{1 /key}

\subsection{GET/key}

Returns a list of all keys

Responses

Code Description Schema

200 The list of keys array[Key]

401 Not authorized String

\subsubsection{2 /keyl\{name\}}

\subsection{GET/key/\{name\}}

Returns a key by name

Responses 
NIST Big DATA INTEROPERABILITY FRAMEWORK: VOLUME 8, REFERENCE ARCHITECTURE INTERFACES

Code Description Schema

200 Returning the information of the key Key

401 Not authorized String

404 The named key could not be found String

Parameters

Name Located in Description Required Schema

name path The name of the key True String

\subsection{PUT /key/\{name\}}

1490 Sets the named key

1491 Responses

Code Description Schema

200 Key updated String

401 Not authorized String

404 The named key could not be found String

1492 Request Body

Located in Description Required Schema

Body The new key to create True Key

\subsection{DELETE /key/\{name\}}

1494 Deletes a key by name

1495 Responses

\begin{tabular}{lll} 
Code & Description & Schema \\
\hline 200 & Deletion successful & String \\
401 & Not authorized & String \\
404 & The named key could not be found & String
\end{tabular}

Parameters

Name Located in Description Required Schema

$\begin{array}{llll}\text { name path } & \text { The name of the key } & \text { True } & \text { String }\end{array}$

\subsubsection{3 publickeystore.yaml}

openapi: "3.0.2"

info:

version: 3.2 .0

$x$-date: $17-06-2019$

$x$-status: defined

title: Public Key Store

description: |- 
NIST Big DATA INTEROPERABILITY FRAMEWORK: Volume 8, REFERENCE ARCHITECTURE INTERFACES

1508

1509

1510

1511

1512

1513

1514

1515

1516

1517

1518

거 1519

1520

1521

1522

1523

1524

1525

1526

1527

1528

1529

1530

1531

1532

1533

1534

1535

1536

1537

1538

1539

1540

1541

1542

1543

1544

1545

1546

1547

1548

1549

1550

1551

1552

1553

1554

1555

1556

1557

1558

1559

1560

1561

1562

1563

1564

1565

1566

1567

1568

1569

1570

1571

1572

1573

1574

1575

1576

public keys.

termsOfService: "https://github.com/cloudmesh/cloudmesh-nist/blob/master/LICENSE.txt" contact:

name: NIST BDRA Interface Subgroup

url: https://cloudmesh-community.github.io/nist

license:

name: Apache 2.0

url: https://github.com/cloudmesh/cloudmesh-nist/blob/master/LICENSE.txt servers:

- url:/cloudmesh/v3

paths:

/key:

get:

tags:

- Key

summary: Returns a list of keys

description: Returns a list of all keys

operationId: cloudmesh.key.list

responses:

'200' :

description: The list of keys

content:

application/json:

schema:

type: array

items:

'401' :

\$ref: '\#/components/schemas/Key'

description: Not authorized

$/$ key/ $\{$ name $\}$ :

get:

tags:

- Key

summary: Returns the named key

description: Returns a key by name

operationId: cloudmesh.key.find_by_name

parameters:

- name: name

in: path

required: true

schema:

type: string

description: The name of the key

responses:

'200' :

description: Returning the information of the key

content:

application/json:

schema:

\$ref: '\#/components/schemas/Key'

'401' :

description: Not authorized

'404' :

description: The named key could not be found

put:

tags:

- Key

summary: Set a key

description: Sets the named key

operationId: cloudmesh.key.add

requestBody:

description: The new key to create

required: true

content:

application/json:

schema:

responses:

\$ref: '\#/components/schemas/Key' 


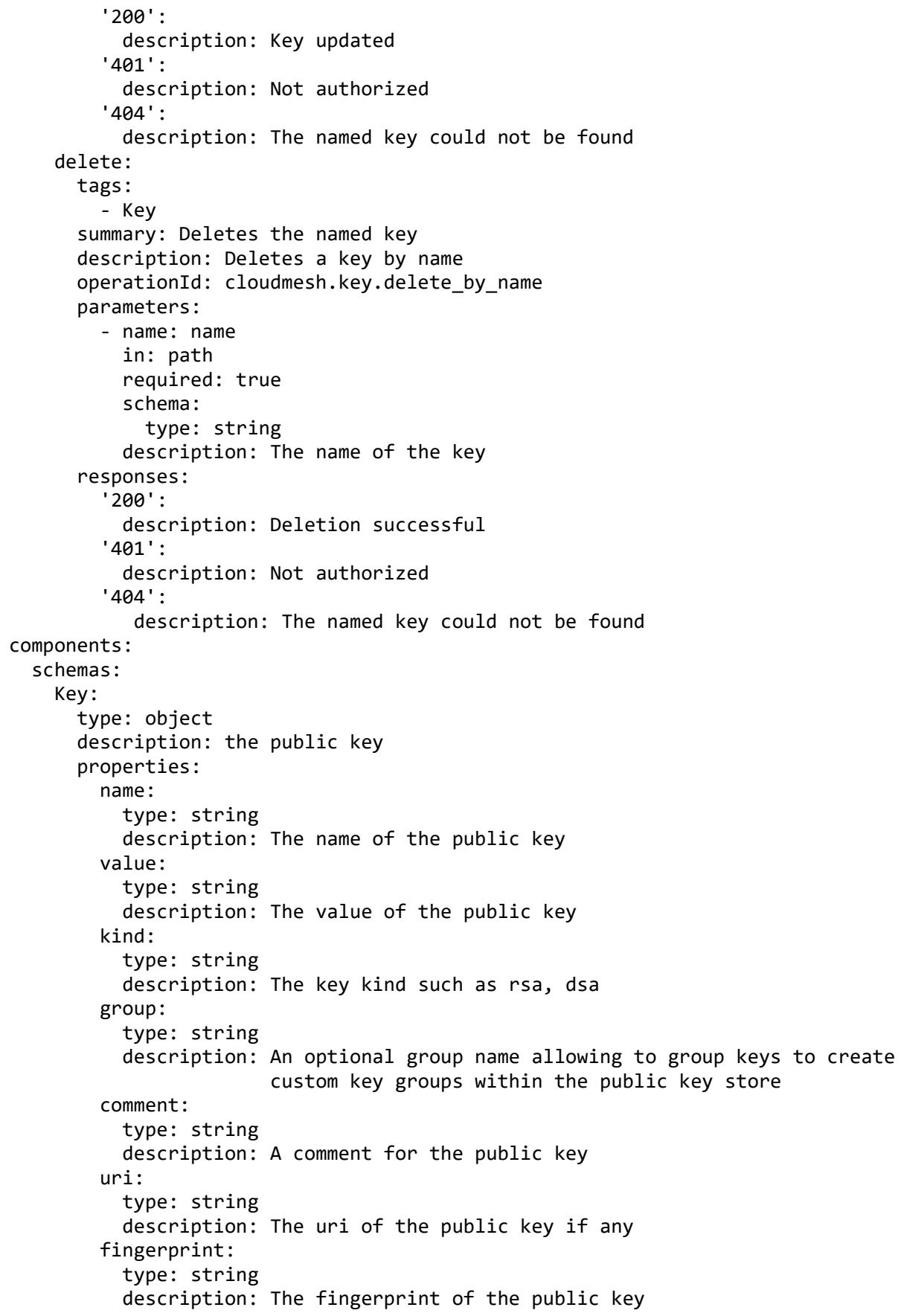

\subsection{VARIABLE, DEFUALT, AND ALIAS}

\subsubsection{ALIAS}

Often a user has the desire to create a custom name for an object. An alias allows for this possibility by users. A name could have one or more aliases. 


\subsubsection{Schema Alias}

\begin{tabular}{lll} 
Property & Type & Description \\
\hline Name & string & The name of the alias \\
Source & string & The original unique object name
\end{tabular}

\subsubsection{Paths}

\begin{tabular}{lll} 
HTTP & Path & Summary \\
\hline get & /alias & Returns a list of aliases \\
get & $/$ alias $/\{$ name $\}$ & Returns the named alias \\
put & $/$ alias $/\{$ name $\}$ & Set an alias \\
delete & $/$ alias $/\{$ name $\}$ & Deletes the named alias
\end{tabular}

$1638 \quad 4.6 .1 .2 .1$ lalias

1639 4.6.1.2.1.1 GET /alias

1640 Returns a list of all aliases

1641 Responses

\begin{tabular}{lll} 
Code & Description & Schema \\
\hline 200 & The list of aliases & array[Alias] \\
400 & No alias found & String \\
401 & Not authorized & String
\end{tabular}

$1642 \quad$ 4.6.1.2.2 lalias/\{name\}

\section{4.6.1.2.2.1 GET /alias/\{name\}}

1644 Returns an alias by name

1645 Responses

\begin{tabular}{lll} 
Code & Description & Schema \\
\hline 200 & Returning the information of the alias & $\underline{\text { Alias }}$ \\
401 & Not authorized & String \\
404 & The named alias could not be found & String
\end{tabular}

1646 Parameters

\begin{tabular}{lllll} 
Name & Located in & Description & Required & Schema \\
\hline name & path & The name of the alias & True & String
\end{tabular}

1647 4.6.1.2.2.2 PUT /alias/\{name\}

1648 Sets the named alias

1649 Responses 
NIST Big DATA INTEROPERABILITY FRAMEWORK: VOLUME 8, REFERENCE ARCHITECTURE INTERFACES

Code Description Schema

200 Alias updated or created String

$401 \quad$ Not authorized String

1650 Request Body

Located in Description Required Schema

Body The new alias to create True $\underline{\text { Alias }}$

\subsection{DELETE /alias/\{name\}}

1652 Deletes an alias by name

1653 Responses

Code Description Schema

200 Deletion successful String

401 Not authorized String

404 The named alias could not be found String

1654

Parameters

Name Located in Description Required Schema

name path The name of the alias True String

\subsubsection{3 alias.yaml}

openapi: '3.0.2'

info:

version: 3.2 .0

x-date: 17-06-2019

$\mathrm{x}$-status: defined

title: Alias

description: |-

Often a user has the desire to create a custom name for an object. An

alias allows to do that while associating a user defined name or

*alias* to a previously used name. The aliases could be shared with other

users. A name could have one or more aliases.

termsOfService: 'https://github.com/cloudmesh/cloudmesh-nist/blob/master/LICENSE.txt' contact:

name: NIST BDRA Interface Subgroup

url: https://cloudmesh-community.github.io/nist/spec/

license:

name: Apache 2.0

url: https://github.com/cloudmesh/cloudmesh-nist/blob/master/LICENSE.txt servers:

- url: /cloudmesh/v3

paths:

/alias:

get:

tags:

- Alias

summary: Returns a list of aliases

description: Returns a list of all aliases

operationId: cloudmesh.alias.list

responses:

'200' :

description: The list of aliases 
NIST Big DATA INTEROPERABILITY FRAMEWORK: Volume 8, REFERENCE ARCHITECTURE INTERFACES

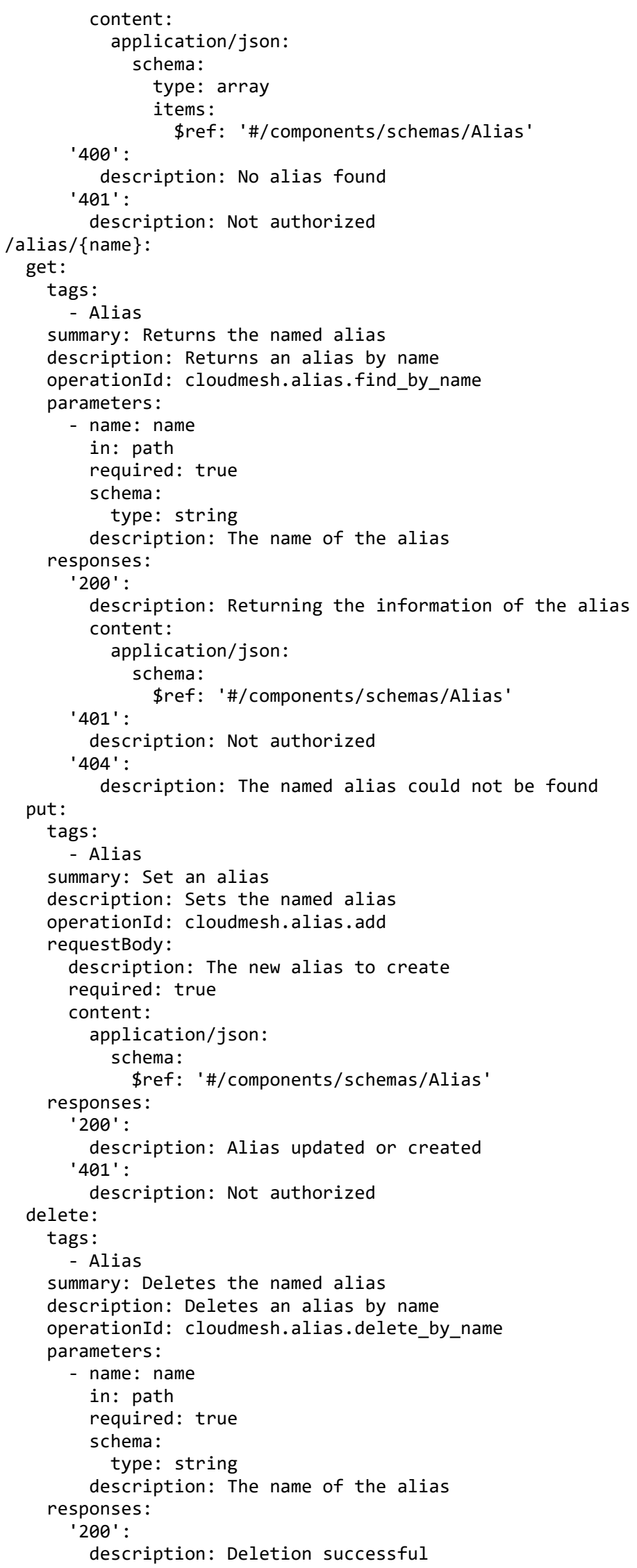


NIST Big DATA INTEROPERABILITY FRAMEWORK: VOLUME 8, REFERENCE ARCHITECTURE INTERFACES

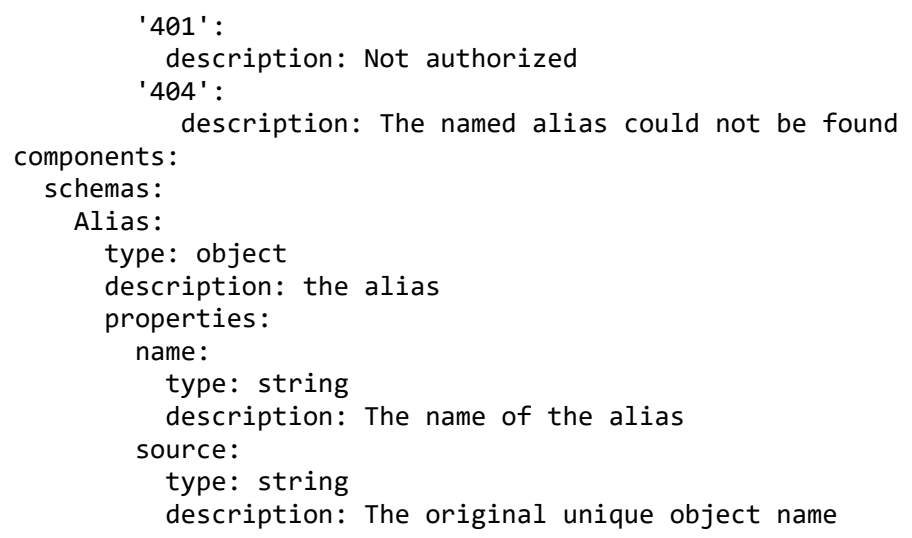

\subsubsection{VARIABLES}

1775 Variables are a simple string key value storage to store simple values. Each variable can have a datatype 1776 so that it can be used for serialization into other formats. Internally they are stored as strings.

\section{4.6.2.1 Schema Variable}

\begin{tabular}{lll} 
Property & Type & Description \\
\hline Name & string & Name of the variable \\
Value & string & Value of the variable \\
Description & string & A description of the variable \\
Datatype & string & The data type of the variable which can be used for serialization
\end{tabular}

$1778 \quad$ 4.6.2.2 Paths

\begin{tabular}{lll} 
HTTP & Path & Summary \\
\hline get & $/$ variable & Returns the variables \\
get & $/$ variable/ $\{$ name $\}$ & Returns the named variable \\
put & $/$ variable $/\{$ name $\}$ & Set the value of a variable \\
delete & $/$ variable $/\{$ name $\}$ & Deletes the named variable
\end{tabular}

$1779 \quad$ 4.6.2.2.1 /variable

1780 4.6.2.2.1.1 GET/variable

1781 Returns the variables

1782 Responses

\begin{tabular}{lll} 
Code & Description & Schema \\
\hline 200 & The list of variables & array[Variable] \\
400 & No variable found & String
\end{tabular}




\subsection{Ivariable/\{name\}}

1784

\subsection{GET/variable/\{name\}}

1785 Returns the named variable

1786 Responses

Code Description Schema

200 Returning the information of the variable Variable

401 Not authorized String

404 The named variable could not be found String

1787 Parameters

\begin{tabular}{lllll} 
Name & Located in & Description & Required & Schema \\
\hline name & path & The name of the variable & True & String
\end{tabular}

1788 4.6.2.2.2.2 PUT /variable/\{name\}

1789 Sets the value of the named variable

1790 Responses

Code Description Schema

200 Variable updated or created String

$400 \quad$ Error updating variable String

401 Not authorized String

1791 Request Body

Located in Description Required Schema

Body The variable and its value $\quad$ True $\quad \underline{\text { Variable }}$

1792 4.6.2.2.2.3 DELETE/variable/\{name\}

1793 Deletes a variable by name

1794 Responses

\begin{tabular}{lll} 
Code & Description & Schema \\
\hline 200 & Deletion successful & String \\
401 & Not authorized & String \\
404 & The named variable could not be found & String
\end{tabular}

1795 Parameters

\begin{tabular}{lllll} 
Name & Located in & Description & Required & Schema \\
\hline name & path & The name of the variable & True & String
\end{tabular}


1796

1797

1798

1799

1800

1801

1802

1803

1804

1805

1806

1807

1808

1809

1810

1811

1812

1813

1814

1815

1816

1817

1818

1819

1820

1821

1822

1823

1824

1825

1826

1827

1828

1829

1830

1831

1832

1833

1834

1835

1836

1837

1838

1839

1840

1841

1842

1843

1844

1845

1846

1847

1848

1849

1850

1851

1852

1853

1854

1855

1856

1857

1858

1859

1860

1861

1862

1863

\subsubsection{3 variables.yaml}

openapi: "3.0.2"

info:

version: 3.2 .0

$x$-date: 17-06-2019

$x$-status: defined

title: Variables

description: |-

Variables are a simple string key value storage to store simple

values. Each variable can have a datatype, so that it can be used for

serialization into other formats. Internally they are stored as strings.

termsOfService: "https://github.com/cloudmesh/cloudmesh-nist/blob/master/LICENSE.txt"

contact:

name: NIST BDRA Interface Subgroup

url: https://cloudmesh-community.github.io/nist/spec/

license:

name: Apache 2.0

url: https://github.com/cloudmesh/cloudmesh-nist/blob/master/LICENSE.txt servers:

- url: /cloudmesh/v3

paths:

/variable:

get:

tags:

- Variable

summary: Returns the variables

description: Returns the variables

operationId: cloudmesh.variable.list

responses:

'200' :

description: The list of variables

content:

application/json:

schema:

type: array

items:

'400' :

\$ref: '\#/components/schemas/Variable'

description: No variable found

/variable/\{name\}:

get:

tags:

- Variable

summary: Returns the named variable

description: Returns the named variable

operationId: cloudmesh.variable.find_by_name

parameters:

- name: name

in: path

required: true

schema:

type: string

description: The name of the variable

responses:

'200' :

description: Returning the information of the variable

content:

application/json:

schema:

\$ref: '\#/components/schemas/Variable'

'401':

description: Not authorized

'404' :

description: The named variable could not be found

put:

tags: 
NIST Big DATA INTEROPERABILITY FRAMEWORK: VOLUME 8, REFERENCE ARCHITECTURE INTERFACES

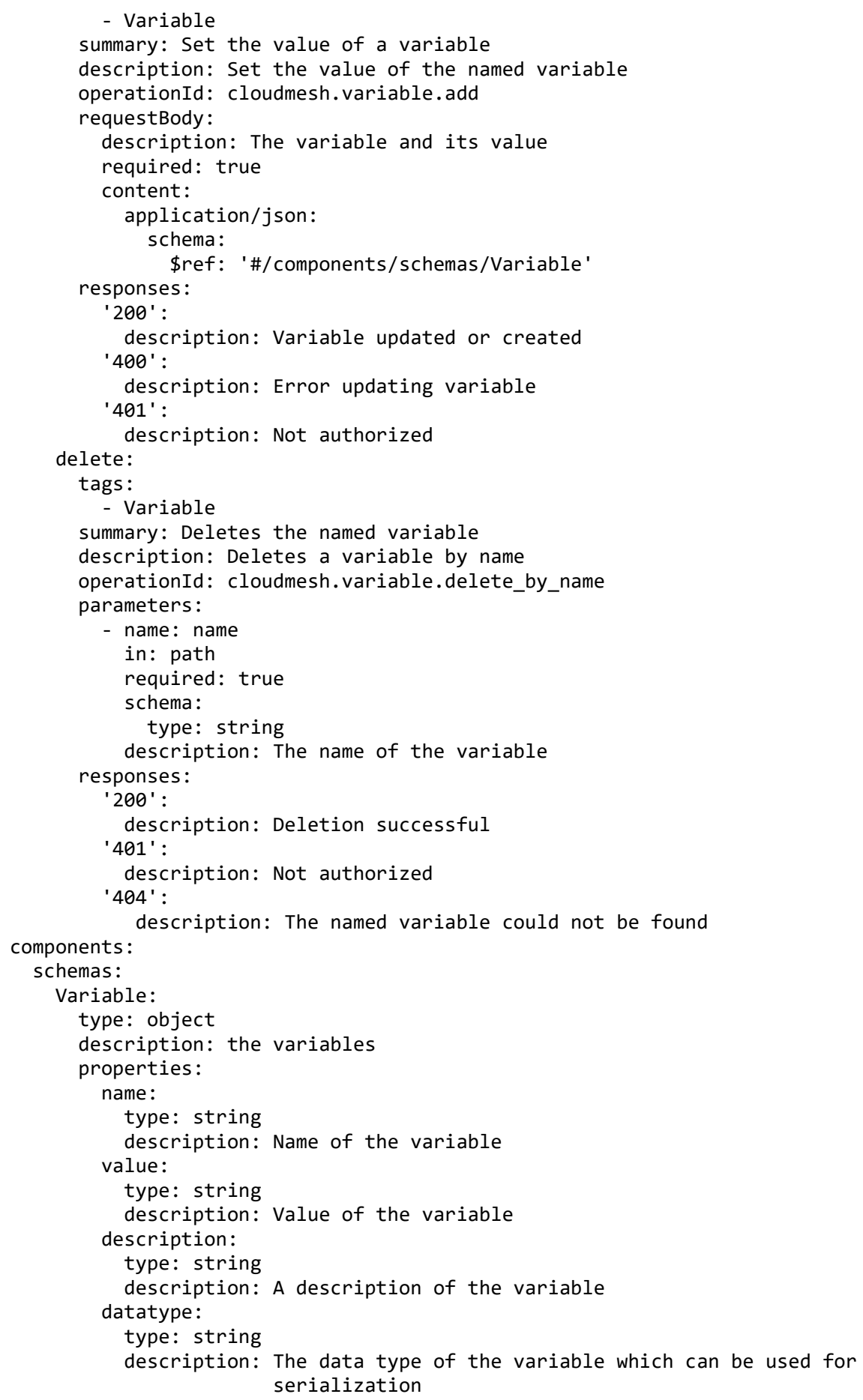

\subsubsection{DEFAULT}

A default is a special variable that has a context associated with it. This allows one to define values that 


\subsubsection{Schema Default}

\begin{tabular}{lll} 
Property & Type & Description \\
\hline name & string & The name of the default \\
value & string & The value of the default \\
context & string & The context of the default
\end{tabular}

\subsubsection{Paths}

\begin{tabular}{lll} 
HTTP & Path & Summary \\
\hline get & $/$ default & Returns a list of defaults \\
get & $/$ default $/\{$ name $\}$ & Returns the named default \\
put & $/$ default $/\{$ name $\}$ & Set a default \\
delete & $/$ default $/\{$ name $\}$ & Deletes the named default
\end{tabular}

$1927 \quad$ 4.6.3.2.1 /default

1928 4.6.3.2.1.1 GET /default

1929 Returns a list of all defaults

1930 Responses

\begin{tabular}{lll} 
Code & Description & Schema \\
\hline 200 & The list of defaults & array[Default \\
401 & Not authorized & String
\end{tabular}

1931 4.6.3.2.2/default/\{name $\}$

1932 4.6.3.2.2.1 GET/default/\{name\}

1933 Returns a default by name

1934 Responses

\begin{tabular}{lll} 
Code & Description & Schema \\
\hline 200 & Returning the information of the default & $\underline{\text { Default }}$ \\
401 & Not authorized & String \\
404 & The named default could not be found & String
\end{tabular}

1935 Parameters

\begin{tabular}{lllll} 
Name & Located in & Description & Required & Schema \\
\hline name & path & The name of the default & True & String
\end{tabular}

1936 4.6.3.2.2.2 PUT /default/\{name\}

1937 Sets the named default 
NIST Big DATA INTEROPERABILITY FRAMEWORK: VOLUME 8, REFERENCE ARCHITECTURE INTERFACES

1938

Responses

Code Description

Schema

200 Default updated or created String

401 Not authorized String

1939 Request Body

\begin{tabular}{llll} 
Located in & Description & Required & Schema \\
\hline Body & The new default to create & True & $\underline{\text { Default }}$
\end{tabular}

4.6.3.2.2.3 DELETE /default/\{name\}

1941 Deletes a default by name

1942 Responses

Code Description Schema

200 Deletion successful String

401 Not authorized String

404 The named default could not be found String

1943 Parameters

\begin{tabular}{lllll} 
Name & Located in & Description & Required & Schema \\
\hline name & path & The name of the default & True & String
\end{tabular}

1944

1945

1946

1947

1948

1949

1950

1951

1952

1953

1954

1955

1956

1957

1958

1959

1960

1961

1962

1963

1964

1965

1966

1967

1968

1969

1970

1971

1972

1973

1974

1975

\subsubsection{3 default.yamI}

openapi: "3.0.2"

info:

version: 3.2 .0

$x$-date: 17-06-2019

$x$-status: defined

title: Default

description: |-

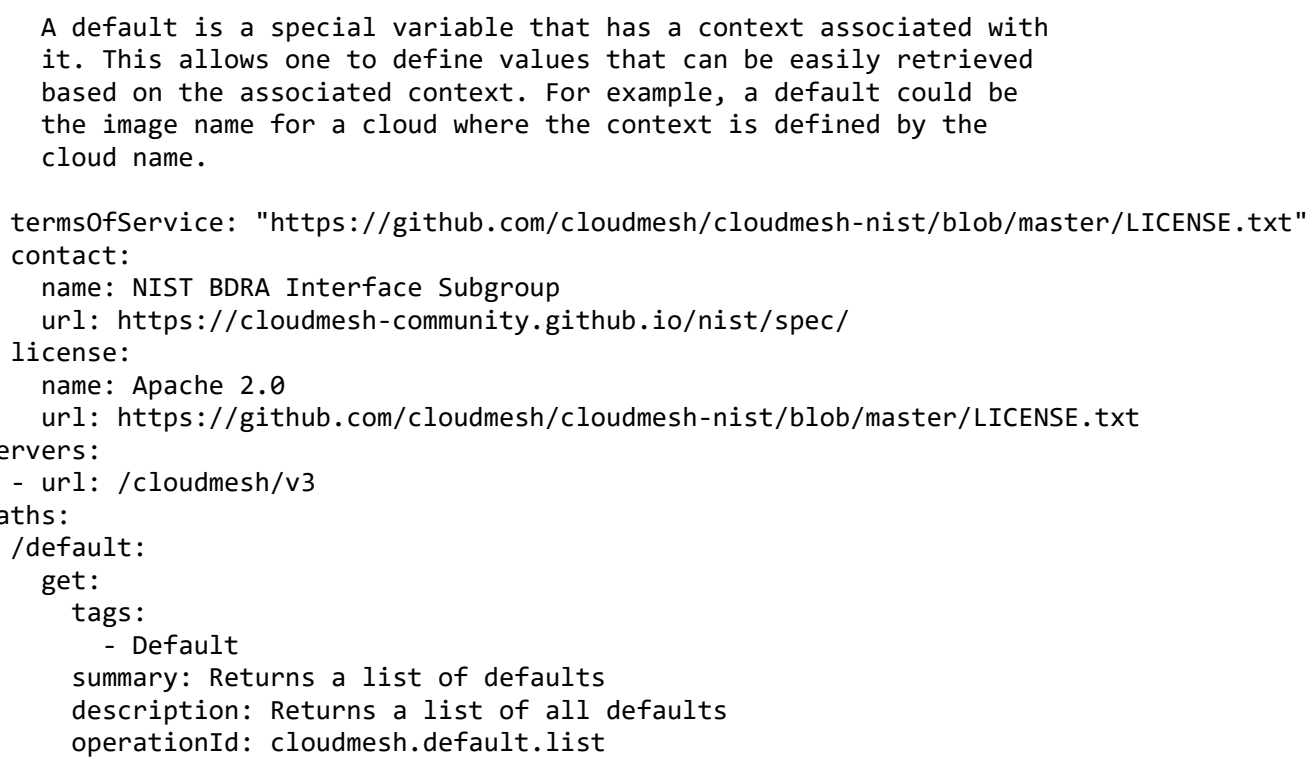


NIST Big DATA INTEROPERABILITY FRAMEWORK: Volume 8, REFERENCE ARCHITECTURE INTERFACES

1976

1977

1978

1979

1980

1981

1982

1983

1984

1985

1986

1987

5. 1988

1989

1990

1991

1992

1993

1994

1995

1996

1997

1998

1999

2000

2001

2002

2003

2004

2005

2006

2007

2008

2009

2010

2011

2012

2013

2014

2015

2016

2017

2018

2019

2020

2021

2022

2023

2024

2025

2026

2027

2028

2029

2030

2031

2032

2033

2034

2035

2036

2037

2038

2039

2040

2041

2042

2043

2044

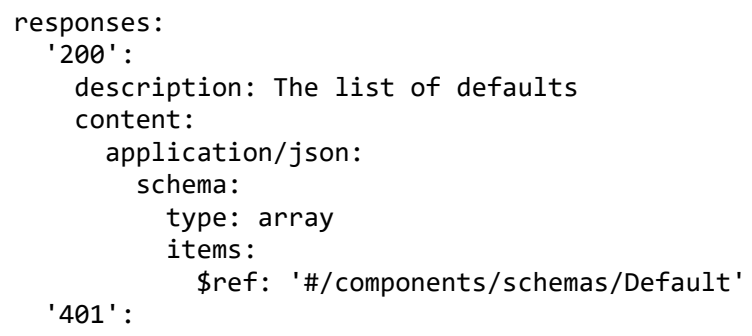




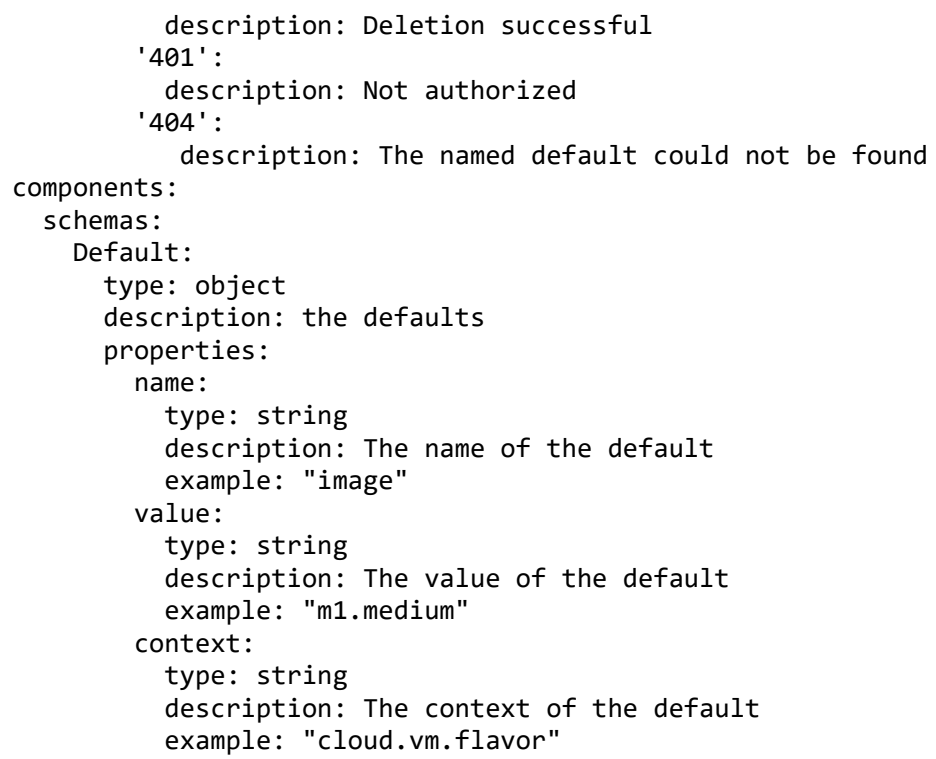

\subsection{DATA MANAGEMENT}

\subsubsection{FILESTORE}

A file store is a resource allowing storage of data as a traditional file. A file store can contain any number of files with additional attributes describing the file. A file store is located on the physical server. It contains access to the content of the file. This contrasts virtual directories that are just pointers to files, which could include files located in different file stores. A virtual directory also does not contain the content of the file, but just a pointer where to find the file.

\subsubsection{Schema File}

\begin{tabular}{|c|c|c|}
\hline Property & Type & Description \\
\hline Name & string & The name of the file \\
\hline Endpoint & string & The location of the file \\
\hline Checksum & string & The checksum of the file \\
\hline Size & integer & $\begin{array}{l}\text { The size of the file in } \\
\text { byte }\end{array}$ \\
\hline Content & string(binary) & the content of the file \\
\hline
\end{tabular}

\subsubsection{Paths}

\begin{tabular}{lll} 
HTTP & Path & Summary \\
\hline get & /file & Returns a list of files in the file store \\
get & $/$ file/ $\{$ name $\}$ & Returns the named file in the file store \\
put & $/$ file/ $\{$ name $\}$ & Uploads a file to the list of files in the file store \\
delete & $/$ file/\{name $\}$ & Deletes the named file in the file store
\end{tabular}


4.7.1.2.1 Ifile

2078

4.7.1.2.1.1 GET /file

2079

Returns a list of all files

2080 Responses

Code Description Schema

200 The list of files array[File]

401 Not authorized String

$2081 \quad 4.7 .1 .2 .2$ Ifile/\{name\}

2082 4.7.1.2.2.1 GET/file/\{name\}

2083 Returns a file by name in the file store

2084 Responses

\begin{tabular}{lll} 
Code & Description & Schema \\
\hline 200 & Returning the information of the file store & $\underline{\text { File }}$ \\
401 & Not authorized & String \\
404 & The named file could not be found & String
\end{tabular}

2085 Parameters

\begin{tabular}{lllll} 
Name & Located in & Description & Required & Schema \\
\hline name & path & The name of the file & True & String
\end{tabular}

2086 4.7.1.2.2.2 PUT /file/\{name\}

2087 Uploads a file to the list of files in the file store

2088 Responses

Code Description Schema

$200 \quad$ File updated or created String

401 Not authorized String

2089 Request Body

Located in Description Required Schema

Body The file to be uploaded True File

2090 4.7.1.2.2.3 DELETE /file/\{name\}

2091 Deletes a file by name 
NIST Big DATA INTEROPERABILITY FRAMEWORK: Volume 8, REFERENCE ARCHITECTURE INTERFACES

2092

Responses

Code Description Schema

200 Deletion successful String

401 Not authorized String

404 The named file could not be found String

Parameters

\begin{tabular}{lllll} 
Name & Located in & Description & Required & Schema \\
\hline name & path & The name of the file & True & String
\end{tabular}

\subsubsection{3 filestore.yaml}

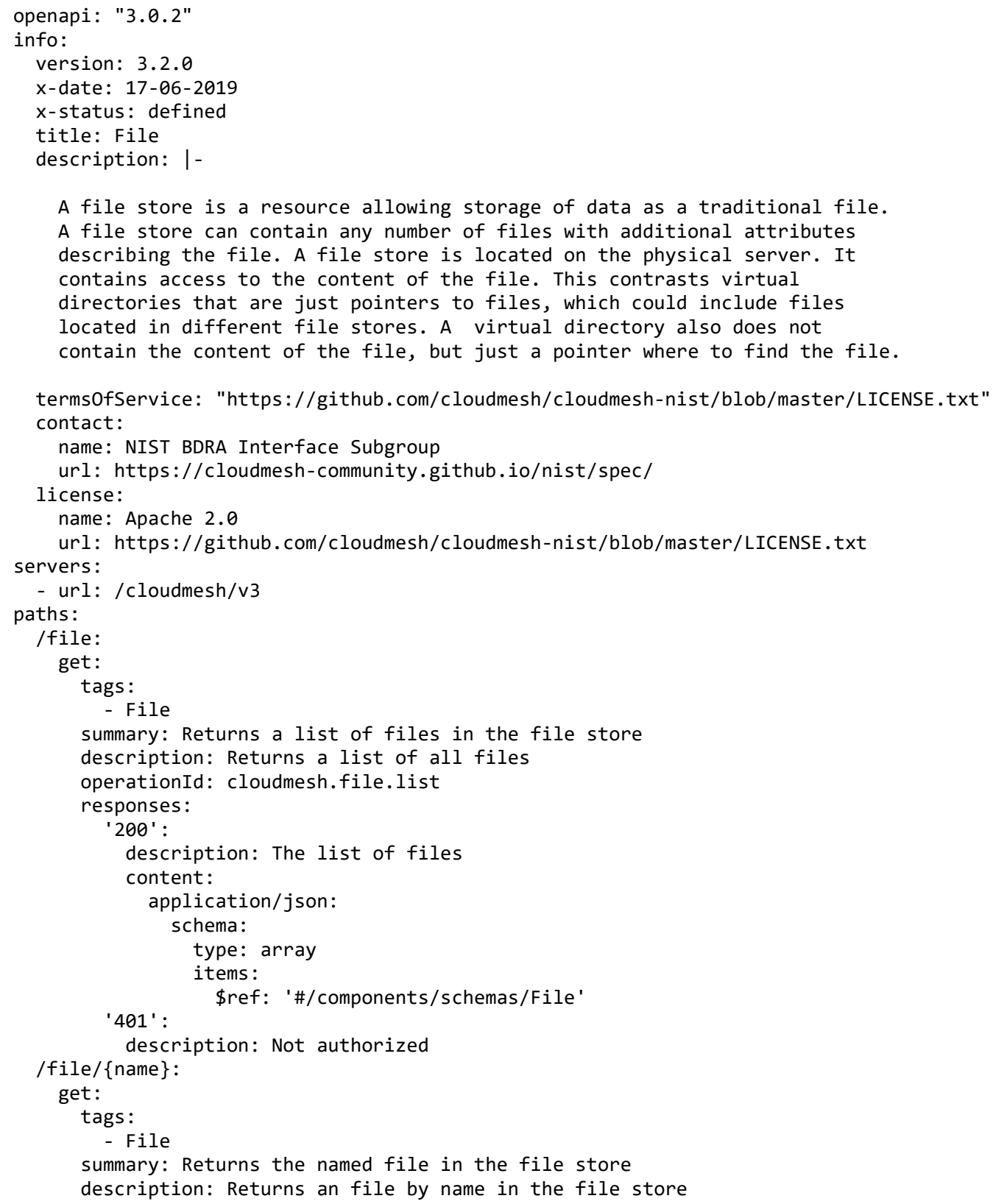


NIST Big DATA INTEROPERABILITY FRAMEWORK: VOLUME 8, REFERENCE ARCHITECTURE INTERFACES

2145

2146

2147

2148

2149

2150

2151

2152

2153

2154

2155

거 2156

ㄷ. 2157

2158

ㅇ 2159

2160

2161

2162

2163

2164

2165

2166

2167

2168

2169

2170

2171

2172

2173

2174

2175

2176

2177

2178

2179

2180

2181

2182

2183

2184

2185

2186

2187

2188

2189

2190

2191

2192

2193

2194

2195

2196

2197

2198

2199

2200

2201

2202

2203

2204

2205

2206

2207

2208

2209

2210

2211

2212

2213

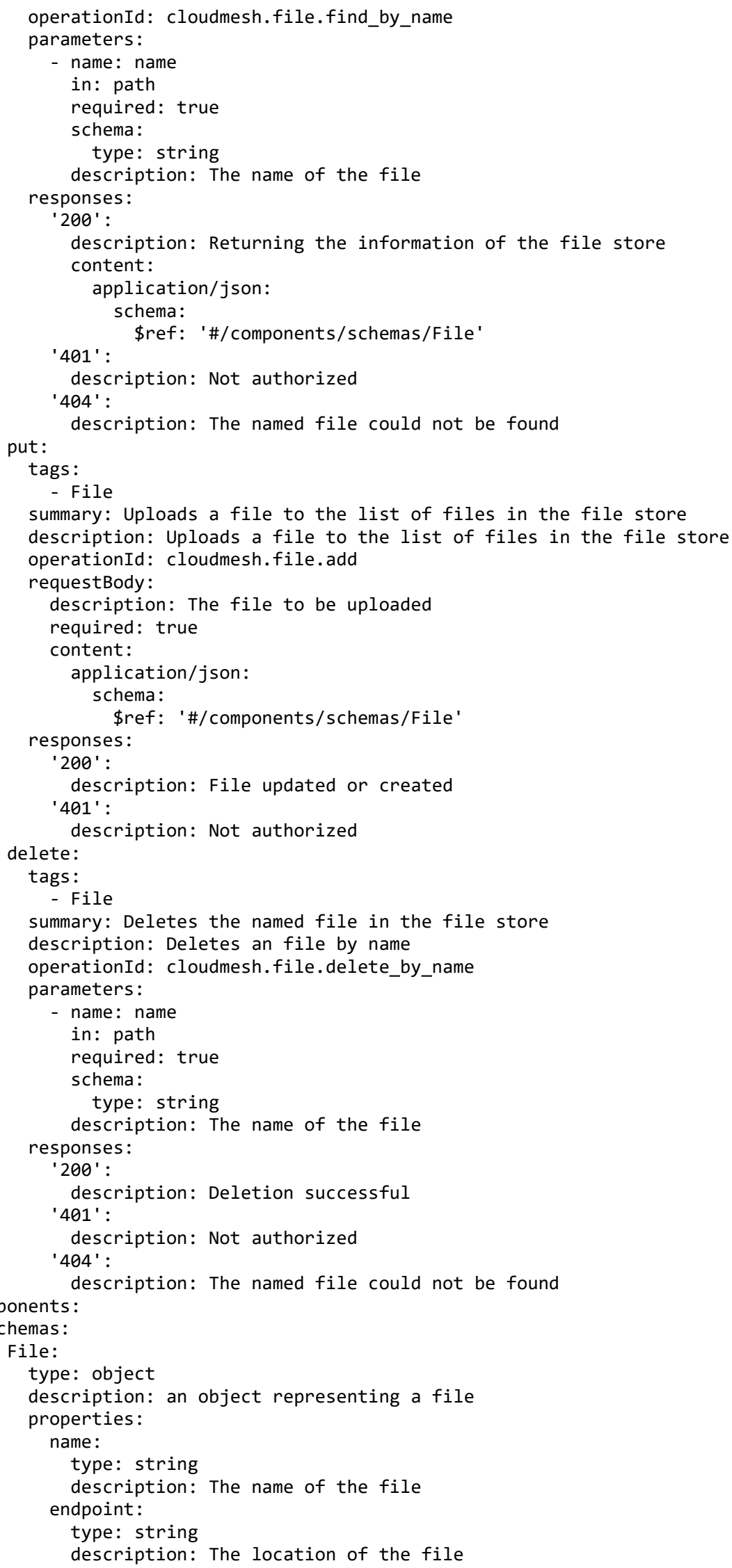




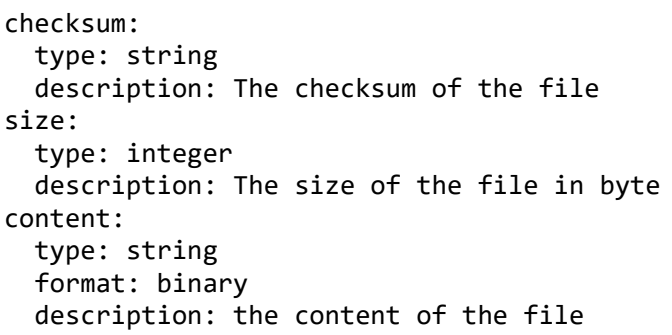

\subsubsection{REPLICA}

In many distributed systems, it is important that a file can be replicated among different systems to provide faster access. It is important to provide a mechanism to trace the pedigree of the file while pointing to its original source. A replica will point to a file in a file store and store the contents in the file store instead of the replica. The replica is just a pointer.

\subsubsection{Schema Replica}

\begin{tabular}{lll} 
Property & Type & Description \\
\hline name & string & The name of the replica \\
filename & string & The original filename \\
endpoint & string & The location of the file \\
checksum & string & The checksum of the file \\
size & integer & The size of the file in byte
\end{tabular}

4.7.2.2 Paths

HTTP Path Summary

get $\quad$ /replica $\quad$ Returns a list of replicas

get $/$ replica/\{name $\}$ Returns the named replica

put $/$ replica/\{name $\}$ Uploads a replica to the list of replicas

delete $/$ replica/\{name $\}$ Deletes the named replica

\subsection{Ireplica}

\subsection{GET/replica}

Returns a list of all replicas

Responses

Code Description

Schema

200 The list of replicas array[Replica]

$401 \quad$ Not authorized String

\subsection{Ireplica/\{name\}}

\subsection{GET/replica/\{name\}}

Returns a replica by name 
NIST Big DATA INTEROPERABILITY FRAMEWORK: VOLUME 8, REFERENCE ARCHITECTURE INTERFACES

Responses

Code Description

Schema

200 Returning the information of the replica Replica

401 Not authorized String

404 The named replica could not be found String

Parameters

\begin{tabular}{lllll} 
Name & Located in & Description & Required & Schema \\
\hline name & path & The name of the replica & True & String
\end{tabular}

2240 4.7.2.2.2.2 PUT /replica/\{name\}

2241 Uploads a replica to the list of replicas

2242 Responses

Code Description Schema

200 Replica updated or created String

401 Not authorized String

2243 Request Body

Located in Description Required Schema

Body $\quad$ The replica to be uploaded True $\quad \underline{\text { Replica }}$

\subsection{DELETE /replica/\{name\}}

2245 Deletes a replica by name

2246 Responses

Code Description Schema

200 Deletion successful String

401 Not authorized String

404 The named replica could not be found String

Parameters

Name Located in Description Required Schema

name path The name of the replica True String

\subsubsection{3 replica.yaml}

openapi: "3.0.2"

info:

version: 3.2 .0

$x$-date: 17-06-2019

$x$-status: defined

title: Replica

description: |-

In many distributed systems, it is important that a file can be replicated among different systems to provide faster access. It is 
NIST Big DATA INTEROPERABILITY FRAMEWORK: Volume 8, REFERENCE ARCHITECTURE INTERFACES

important to provide a mechanism to trace the pedigree of the file while pointing to its original source. A replica will point to a file in a file store and store the contents in the file store instead of the replica. The replica is just a pointer. termsOfService: "https://github.com/cloudmesh/cloudmesh-nist/blob/master/LICENSE.txt" contact:

name: NIST BDRA Interface Subgroup

url: https://cloudmesh-community.github.io/nist/spec/

license:

name: Apache 2.0

url: https://github.com/cloudmesh/cloudmesh-nist/blob/master/LICENSE.txt servers:

- url: /cloudmesh/v3 paths:

/replica:

get:

tags:

- Replica

summary: Returns a list of replicas

description: Returns a list of all replicas

operationId: cloudmesh.replica.list

responses:

'200' :

description: The list of replicas

content:

application/json:

schema:

type: array

items:

' 401 ' :

\$ref: '\#/components/schemas/Replica'

description: Not authorized

/replica/\{name\}:

get:

tags:

- Replica

summary: Returns the named replica

description: Returns an replica by name

operationId: cloudmesh.replica.find_by_name

parameters:

- name: name

in: path

required: true

schema:

type: string

description: The name of the replica responses:

'200' :

description: Returning the information of the replica

content:

application/json:

schema:

\$ref: '\#/components/schemas/Replica'

' 401 ' :

description: Not authorized

'404' :

put:

description: The named replica could not be found

tags:

- Replica

summary: Uploads a replica to the list of replicas

description: Uploads a replica to the list of replicas

operationId: cloudmesh.replica.add

requestBody:

description: The replica to be uploaded

required: true

content:

application/json:

schema: 
NIST Big DATA INTEROPERABILITY FRAMEWORK: Volume 8, REFERENCE ARCHITECTURE INTERFACES

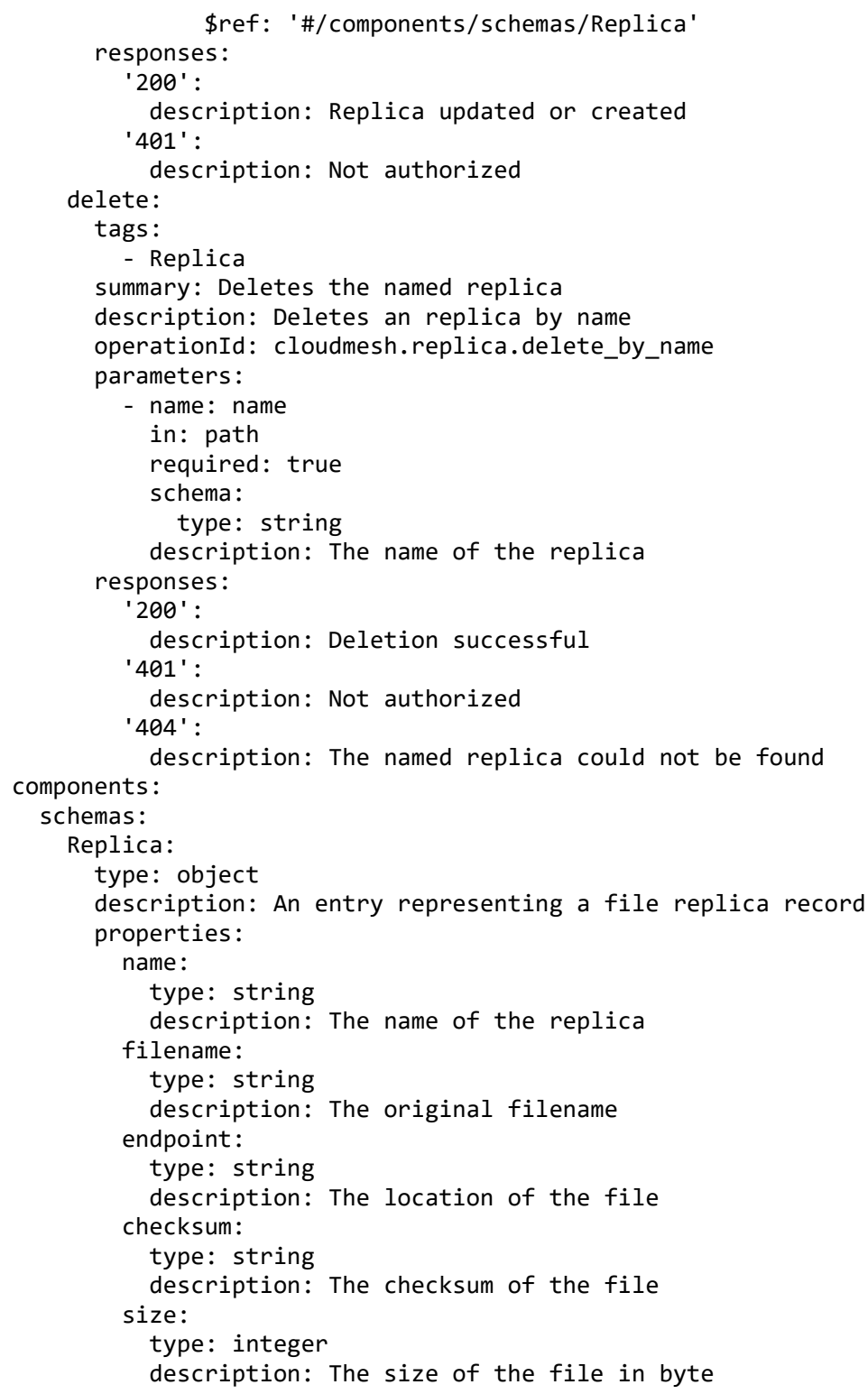

\subsubsection{DATABASE}

The database specification allows registration of a database and performance of elementary operations to use the database. Distinguished below are actions related to the registration of a database, the adding of a schema, the insertion of data, and the query of data. The database is defined by the name of an endpoint (e.g., host, port) and the protocol used (e.g., SQL, NoSQL, graph-based databases).

\subsubsection{Schema Database}

\begin{tabular}{lll} 
Property & Type & Description \\
\hline name & string & Name of the database \\
description & string & Description of the database \\
endpoint & string & Endpoint of the database \\
kind & string & the kind of the database
\end{tabular}




\subsubsection{Schema Schema}

\begin{tabular}{lll} 
Property & Type & Description \\
\hline name & string & Name of the database \\
description & string & Description of the database \\
kind & string & The kind of the definition \\
content & string & The schema associated with the table or collection
\end{tabular}

\subsubsection{Schema Record}

\begin{tabular}{lll} 
Property & Type & Description \\
\hline status & string & The status of the return \\
result & string & The result of the query in json string format
\end{tabular}

\section{4.7.3.4 Schema Query}

\begin{tabular}{lll} 
Property & Type & Description \\
\hline status & string & The query string
\end{tabular}

2384 4.7.3.5 Paths

\begin{tabular}{lll} 
HTTP & Path & Summary \\
\hline get & /database & Returns all databases \\
get & $/$ database $/\{$ name $\} /$ schema & Get the list of the schema \\
put & $/$ database $/\{$ name $/$ schema & Upload a schema \\
delete & $/$ database $/\{$ name $/$ schema & Deletes a database from the list of databases \\
get & $/$ database $/\{$ name $\}$ & Query the named database \\
put & $/$ database $/\{$ name $\}$ & add data to the table or collection \\
delete & $/$ database $/\{$ name $\}$ & Delete the objects matching the query
\end{tabular}

\section{4.7.3.5.1 /database}

2386 4.7.3.5.1.1 GET/database

2387 Returns all databases 
NIST Big DATA INTEROPERABILITY FRAMEWORK: VOLUME 8, REFERENCE ARCHITECTURE INTERFACES

2388

Responses

\begin{tabular}{lll} 
Code & Description & Schema \\
\hline 200 & List of databases & array[Database] \\
401 & Not authorized & String \\
404 & Named database not found & String
\end{tabular}

\section{4.7.3.5.2 Idatabase/\{name\}/schema}

2390 4.7.3.5.2.1 GET/database/\{name\}/schema

2391 Responses

Code Description Schema

200 successfully returned the schema array[Schema]

401 Not authorized String

404 Named database not found String

2392 Parameters

\begin{tabular}{lllll} 
Name & Located in & Description & Required & Schema \\
\hline name & path & Name of the schema & True & String
\end{tabular}

2393 4.7.3.5.2.2 PUT /database/\{name\}/schema

2394 Responses

\begin{tabular}{lll} 
Code & Description & Schema \\
\hline 200 & successfully returned the list & Schema \\
401 & Not authorized & String \\
404 & Named database not found & String
\end{tabular}

2395 Parameters

\begin{tabular}{lllll} 
Name & Located in & Description & Required & Schema \\
\hline name & path & Name of the database & True & String
\end{tabular}

2396 4.7.3.5.2.3 DELETE /database/\{name\}/schema

2397 Deletes a database from the list of databases

2398 Responses

\begin{tabular}{lll} 
Code & Description & Schema \\
\hline 200 & Deletion successful & String \\
401 & Not authorized & String \\
404 & Named database not found & String
\end{tabular}


NIST Big DATA INTEROPERABILITY FRAMEWORK: VOLUME 8, REFERENCE ARCHITECTURE INTERFACES

Parameters

\begin{tabular}{lllll} 
Name & Located in & Description & Required & Schema \\
\hline name & path & Name of the database & True & String
\end{tabular}

$2400 \quad$ 4.7.3.5.3 /database/\{name\}

2401 4.7.3.5.3.1 GET/database/\{name\}

2402 Query the named database

2403 Responses

\begin{tabular}{|c|c|c|c|c|c|}
\hline \multirow{2}{*}{$\begin{array}{l}\text { Code } \\
200\end{array}$} & \multicolumn{2}{|l|}{ Description } & \multicolumn{2}{|c|}{ Schema } & \\
\hline & \multicolumn{2}{|c|}{ Successful query } & \multicolumn{3}{|c|}{ array[Record] } \\
\hline 401 & \multicolumn{2}{|c|}{ Not authorized } & \multicolumn{3}{|l|}{ String } \\
\hline 404 & \multicolumn{2}{|c|}{ Named database not found } & \multicolumn{3}{|l|}{ String } \\
\hline \multicolumn{6}{|c|}{ Parameters } \\
\hline Name & Located in & Description & & Required & Schema \\
\hline name & path & Name of the & atabase & True & String \\
\hline query & query & Database Que & & True & Query \\
\hline
\end{tabular}

2405 4.7.3.5.3.2 PUT /database/\{name\}

2406 Responses

\begin{tabular}{lll} 
Code & Description & Schema \\
\hline 200 & successfully uploaded & $\underline{\text { Record }}$ \\
401 & Not authorized & String \\
404 & Named database not found & String
\end{tabular}

2407 Parameters

\begin{tabular}{lllll} 
Name & Located in & Description & Required & Schema \\
\hline name & path & Name of the database & True & String
\end{tabular}

2408 Request Body

\begin{tabular}{llll} 
Located in & Description & Required & Schema \\
\hline Body & Record to be uploaded & True & $\underline{\text { Record }}$
\end{tabular}


NIST Big DATA INTEROPERABILITY FRAMEWORK: Volume 8, REFERENCE ARCHITECTURE INTERFACES

\subsection{DELETE /database/\{name\}}

2410 Responses

Code Description

Schema

200 Successful query

array[Record]

401 Not authorized

String

404 Named database not found String

Parameters

Name Located in Description Required Schema

$\begin{array}{lllll}\text { name } & \text { path } & \text { Name of the database } & \text { True } & \text { String } \\ \text { query } & \text { query } & \text { Database Query } & \text { True } & \text { Query }\end{array}$

4.7.3.6 database.yaml

openapi: "3.0.2"

info:

version: 3.2 .0

x-date: 17-06-2019

$x$-status: defined

title: Database

description: |-

The database specification allows to register a database and perform

elementary operations to use this database. We distinguish actions

related to the registration, the adding of a schema, the insertion of

data and the query of data. The data base is defined by a name an endpoint

(e.g., host, port), and a protocol used (e.g., SQL, NoSQL, graph-based databases)

termsOfService: "https://github.com/cloudmesh/cloudmesh-nist/blob/master/LICENSE.txt"

contact:

name: NIST BDRA Interface Subgroup

url: https://cloudmesh-community.github.io/nist/spec/

license:

name: Apache 2.0

url: https://github.com/cloudmesh/cloudmesh-nist/blob/master/LICENSE.txt

servers:

- url:/cloudmesh/v3

paths:

/database:

get:

tags:

- "Database Registry"

summary: Returns all databases

description: Returns all databases

operationId: cloudmesh.database.get

responses:

'200':

description: List of databases

content:

application/json:

schema:

type: array

items:

'401' :

\$ref: "\#/components/schemas/Database"

2453

2454

description: Not authorized

'404' :

description: Named database not found

/database/\{name\}/schema: 
NIST Big DATA INTEROPERABILITY FRAMEWORK: Volume 8, REFERENCE ARCHITECTURE INTERFACES

2459

2460

2461

2462

2463

2464

2465

2466

2467

2468

2469

거 2470

2471

2472

2473

2474

2475

2476

2477

2478

2479

2480

2481

2482

2483

2484

2485

2486

2487

2488

2489

2490

2491

2492

2493

2494

2495

2496

2497

2498

2499

2500

2501

2502

2503

2504

2505

2506

2507

2508

2509

2510

2511

2512

2513

2514

2515

2516

2517

2518

2519

2520

2521

2522

2523

2524

2525

2526

2527

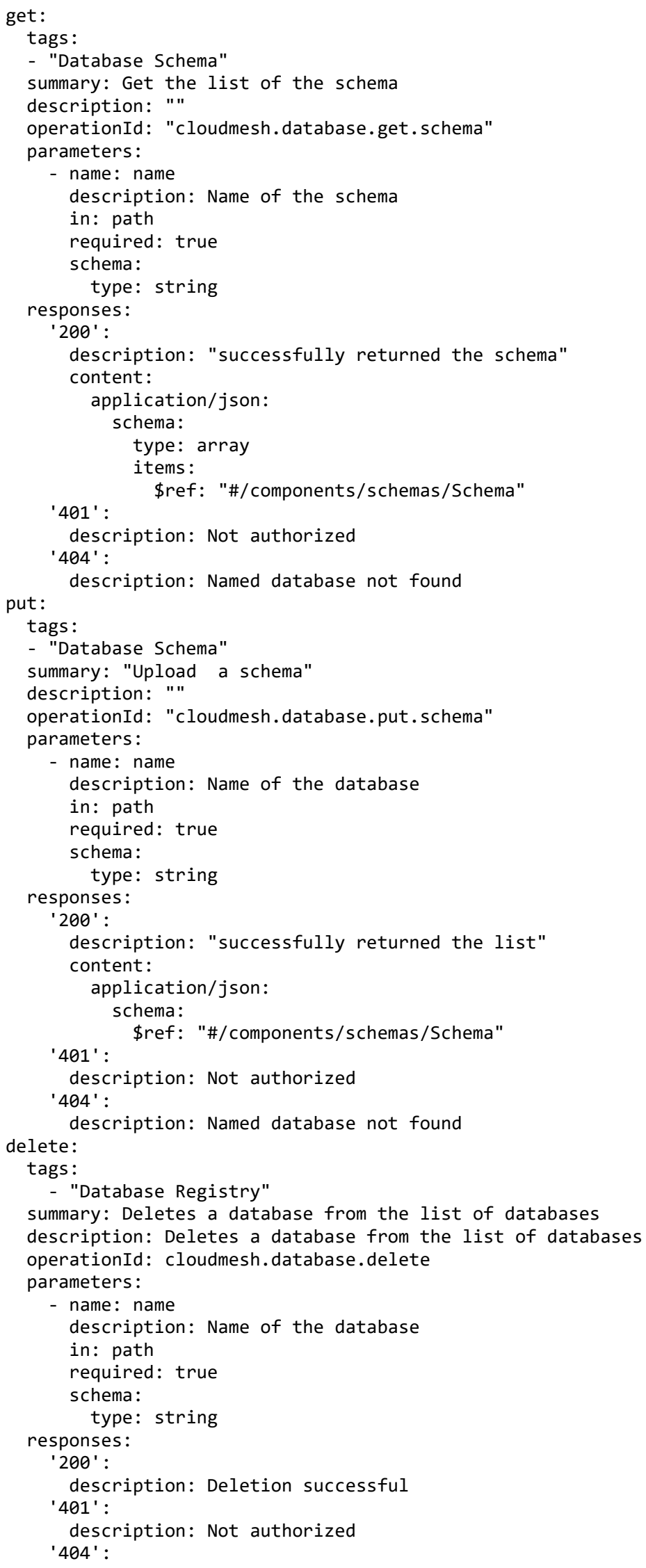




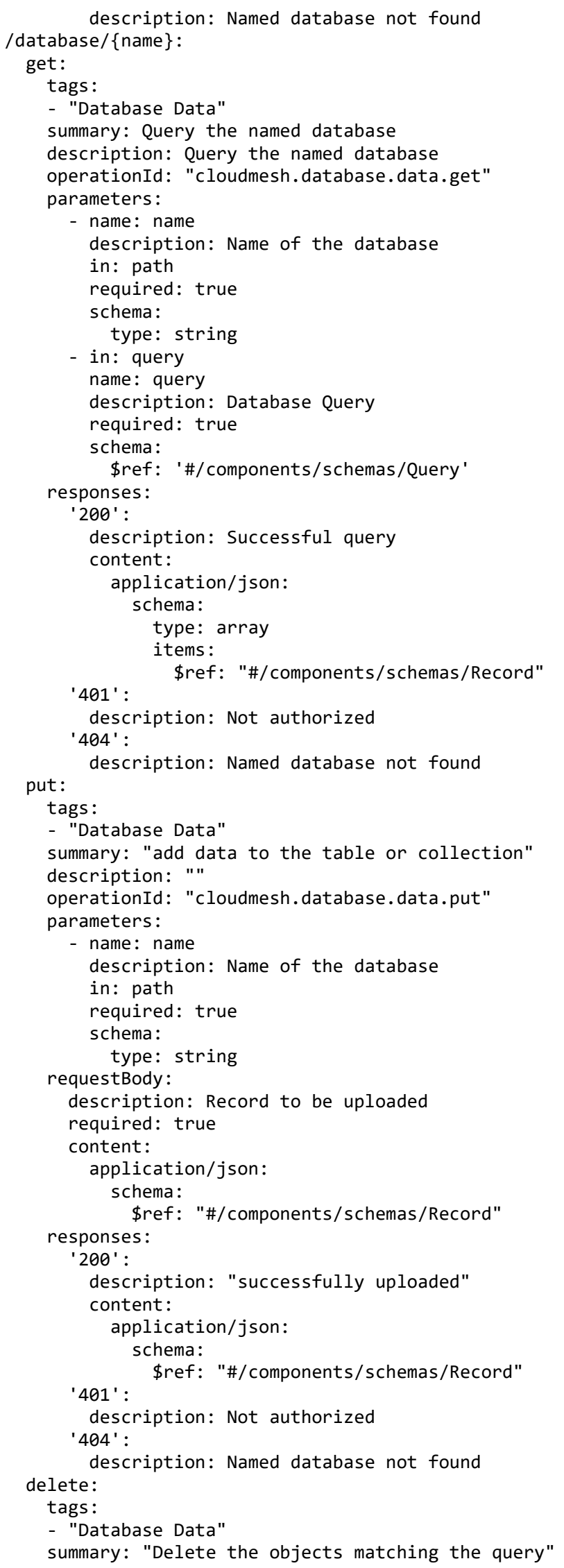


NIST Big DATA INTEROPERABILITY FRAMEWORK: Volume 8, REFERENCE ARCHITECTURE INTERFACES

2597
2598
2599
2600
2601
2602
2603
2604
2605
2606
2607
2608
2609
2610
2611
2612
2613
2614
2615
2616
2617
2618
2619
2620
2621
2622
2623
2624
2625
2626
2627
2628
2629
2630
2631
2632
2633
2634
2635
2636
2637
2638
2639
2640
2641
2642
2643
2644
2645
2646
2647
2648
2649
2650
2651
2652
2653
2654
2655
2656
2657
2658
2659
2660
2661
2662
2663
2664
2665

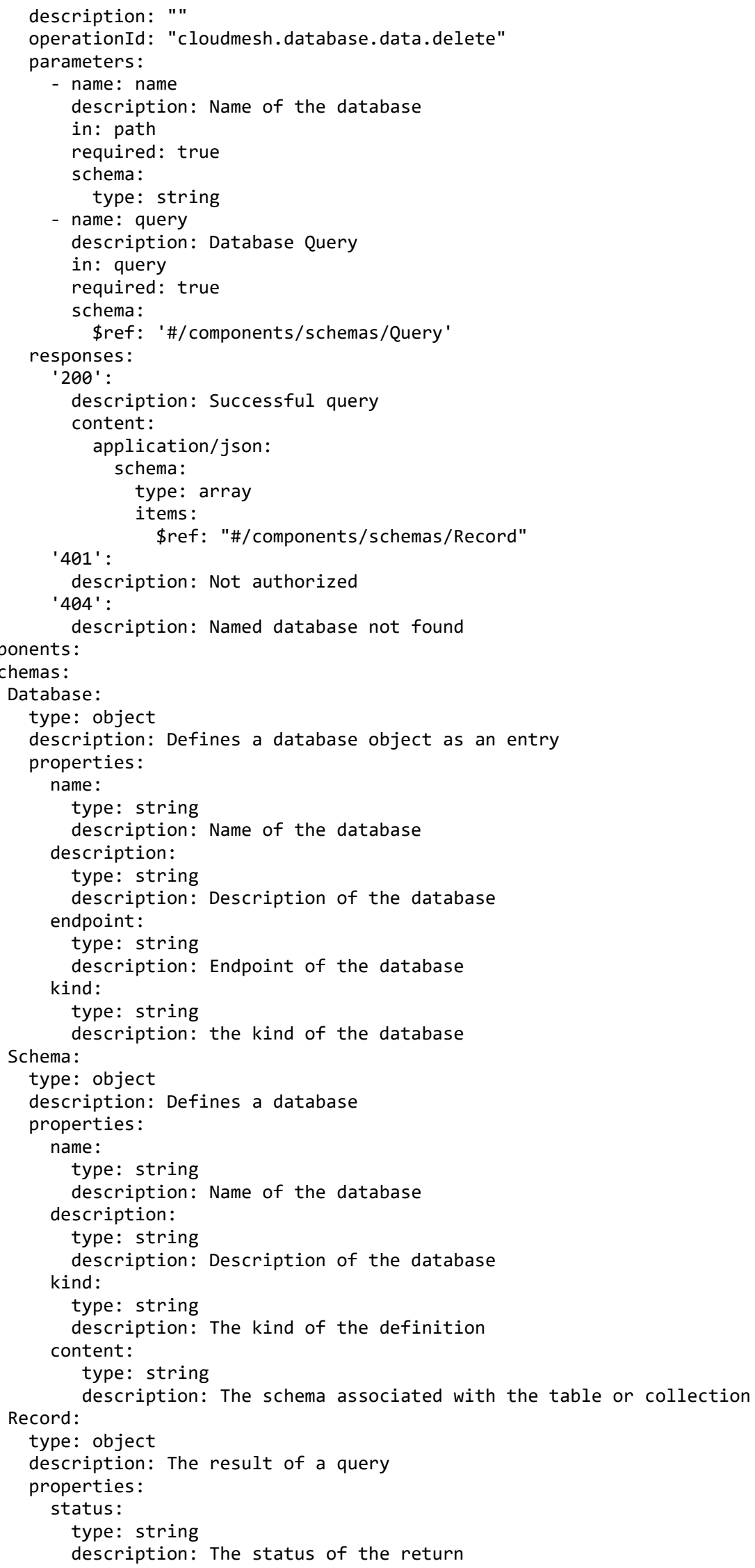


NIST Big DATA INTEROPERABILITY FRAMEWORK: VOLUME 8, REFERENCE ARCHITECTURE INTERFACES

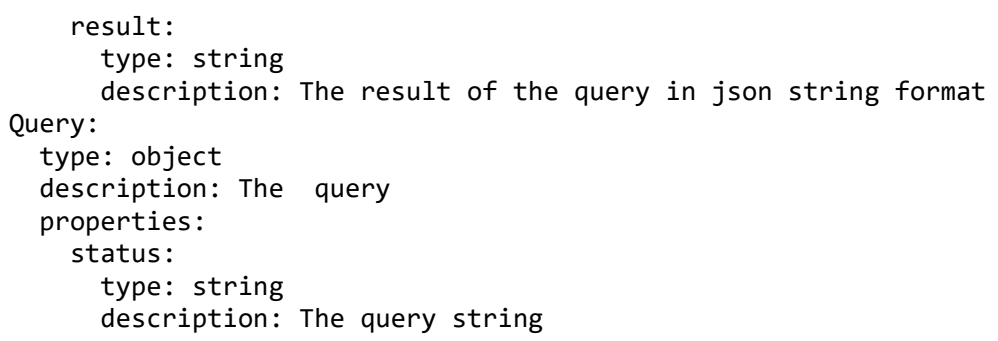

\subsubsection{VirTual DiREctory}

A virtual directory is a collection of files, replicas, streams, or other virtual directories.

\subsubsection{Schema Virtualdirectory}

\begin{tabular}{lll} 
Property & Type & Description \\
\hline name & string & The name of the virtual directory \\
description & string & Description of the virtual directory \\
host & string & Remote host of the virtual directory \\
location & string & Remote location, e.g., a directory with full path on a host \\
protocol & string & Access protocol (e.g. HTTP, FTP, SSH, etc.) \\
credential & object & Credential to access
\end{tabular}

2679 4.7.4.2 Paths

\begin{tabular}{lll} 
HTTP & Path & Summary \\
\hline get & $/$ virtualdirectory & Returns a list of virtual directories \\
get & $/$ virtualdirectory $/\{$ name $\}$ & Returns the named virtual directory \\
put & $/$ virtualdirectory $/\{$ name $\}$ & $\begin{array}{l}\text { Uploads a virtual directory to the list of virtual } \\
\text { directories }\end{array}$ \\
delete & $/$ virtualdirectory $/\{$ name $\}$ & Deletes the named virtual directory \\
get & $/$ virtualdirectory $/\{$ name $\} /\{$ filename $\}$ & Returns the specific file of that virtual directory \\
put & $/$ virtualdirectory $/\{$ name $/\{$ filename $\}$ & Updates or adds a virtual file in the virtual directory \\
delete & $/$ virtualdirectory $/\{$ name $\} /$ filename $\}$ & Delete an user in the virtual directory
\end{tabular}

$2680 \quad$ 4.7.4.2.1 /virtualdirectory

2681 4.7.4.2.1.1 GET/virtualdirectory

2682 Returns a list of all virtual directories 
NIST Big DATA INTEROPERABILITY FRAMEWORK: VOLUME 8, REFERENCE ARCHITECTURE INTERFACES

2683

Responses

\begin{tabular}{lll} 
Code & Description & Schema \\
\hline 200 & The list of virtual directories & array[Virtualdirectory] \\
401 & Not authorized & String
\end{tabular}

$2684 \quad$ 4.7.4.2.2 Ivirtualdirectory/\{name\}

2685 4.7.4.2.2.1 GET/virtualdirectory/\{name\}

2686 Returns a virtual directory by name

2687 Responses

\begin{tabular}{|c|c|c|c|c|}
\hline Code & \multicolumn{2}{|l|}{ Description } & \multicolumn{2}{|c|}{ Schema } \\
\hline 200 & Returning th & e information of the virtual directory & \multicolumn{2}{|c|}{$\underline{\text { Virtualdirectory }}$} \\
\hline 401 & Not authoriz & & \multicolumn{2}{|l|}{ String } \\
\hline 404 & The named & irtual directory could not be found & \multicolumn{2}{|l|}{ String } \\
\hline \multicolumn{5}{|c|}{ Parameters } \\
\hline Name & Located in & Description & Required & Schema \\
\hline name & path & The name of the virtual directory & True & String \\
\hline
\end{tabular}

2689 4.7.4.2.2.2 PUT/virtualdirectory/\{name\}

2690 Uploads a virtual directory to the list of virtual directories

2691 Responses

200 Virtual directory updated or created String

401 Not authorized String

404 The named virtual directory could not be found String

2692 Request Body

Located in Description Required Schema

Body The virtual directory to be uploaded True $\quad$ Virtualdirectory

2693 4.7.4.2.2.3 DELETE /virtualdirectory/\{name\}

2694 Deletes a virtual directory by name

2695 Responses

\begin{tabular}{lll} 
Code & Description & Schema \\
\hline 200 & Deletion successful & String \\
401 & Not authorized & String \\
404 & The named virtual directory could not be found & String
\end{tabular}


NIST Big DATA INTEROPERABILITY FRAMEWORK: VOLUME 8, REFERENCE ARCHITECTURE INTERFACES

2696

Parameters

\begin{tabular}{lllll} 
Name & Located in & Description & Required & Schema \\
\hline name & path & The name of the virtual directory & True & String
\end{tabular}

$2697 \quad$ 4.7.4.2.3 /virtualdirectory/\{name\}/\{filename\}

2698 4.7.4.2.3.1 GET /virtualdirectory/\{name\}/\{filename\}

2699 Returns the specific file of that virtual directory

2700 Responses

\begin{tabular}{lll} 
Code & Description & Schema \\
\hline 200 & upload successful & $\underline{\text { File }}$ \\
401 & Not authorized & String \\
404 & The named virtual directory or file could not be found & String
\end{tabular}

2701 Parameters

\begin{tabular}{lllll} 
Name & Located in & Description & Required & Schema \\
\hline name & path & The name of the virtual directory & True & String \\
filename & path & The filename & True & String
\end{tabular}

2702 4.7.4.2.3.2 PUT /virtualdirectory/\{name\}/\{filename\}

2703 Updates or adds a virtual file in the virtual directory

2704 Responses

Code Description Schema

200 User added successfully String

401 Not authorized String

2705 Parameters

\begin{tabular}{lllll} 
Name & Located in & Description & Required & Schema \\
\hline name & path & The name of the virtual directory & True & String \\
filename & path & The filename & True & String
\end{tabular}

2706 Request Body

Located in Description Required Schema

Body The user to be uploaded True $\underline{\text { File }}$

2707 4.7.4.2.3.3 DELETE /virtualdirectory/\{name\}/\{filename\}

2708 Delete a user in the virtual directory 
NIST Big DATA INTEROPERABILITY FRAMEWORK: Volume 8, REFERENCE ARCHITECTURE INTERFACES

Responses

Code Description

Schema

$200 \quad$ Deletion successful

String

$401 \quad$ Not authorized

String

404 The named virtual directory or file could not be found String

Parameters

\begin{tabular}{lllll} 
Name & Located in & Description & Required & Schema \\
\hline name & path & The name of the virtual directory & True & String \\
filename & path & The filename & True & String
\end{tabular}

2711

2712

2713

2714

2715

2716

2717

2718

2719

2720

2721

2722

2723

2724

2725

2726

2727

2728

2729

2730

2731

2732

2733

2734

2735

2736

2737

2738

2739

2740

2741

2742

2743

2744

2745

2746

2747

2748

2749

2750

2751

2752

2753

2754

2755

2756

2757

2758

2759

\subsubsection{3 virtualdirectory.yaml}

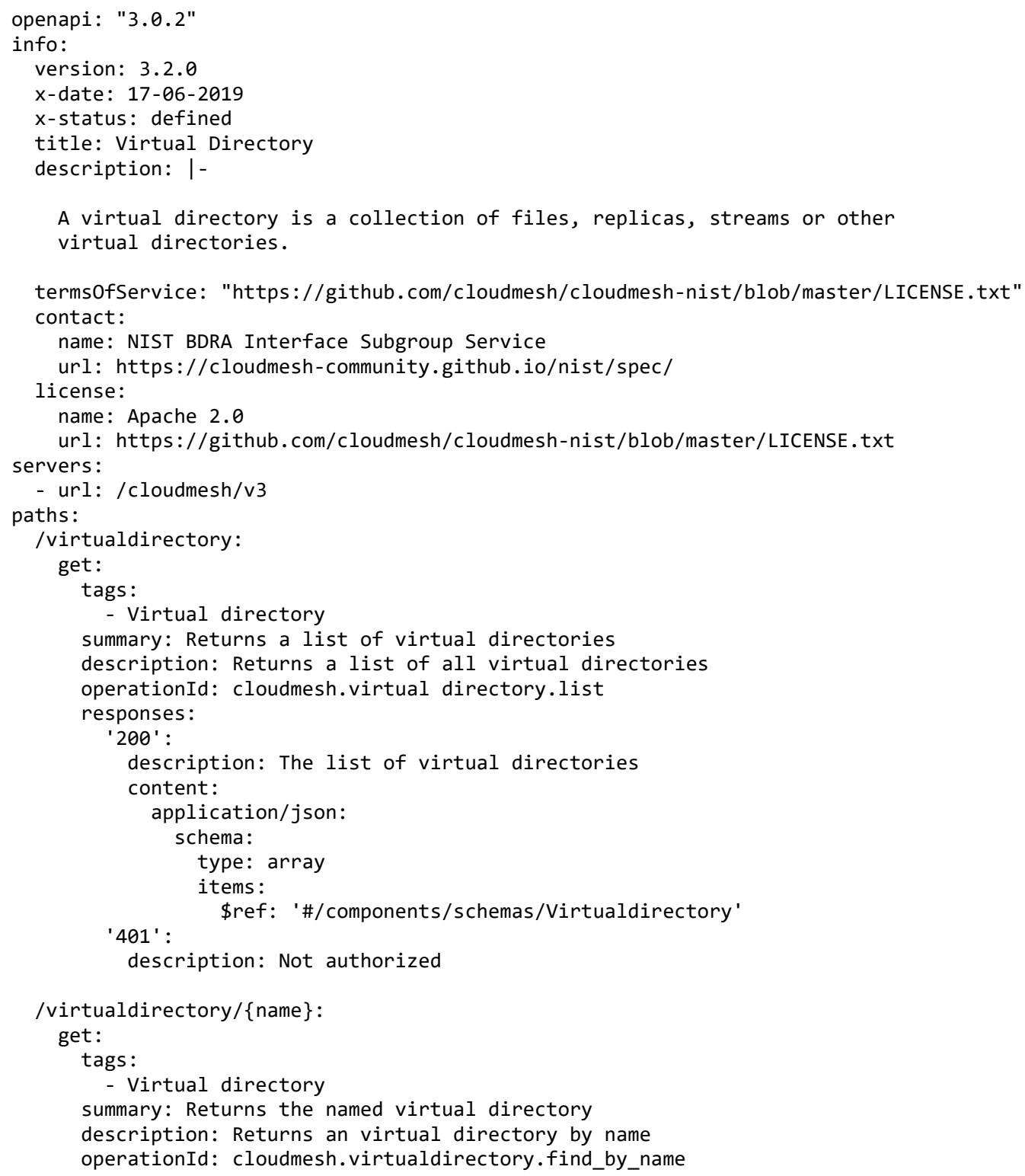


NIST Big DATA INTEROPERABILITY FRAMEWORK: Volume 8, REFERENCE ARCHITECTURE INTERFACES

2760

2761

2762

2763

2764

2765

2766

2767

2768

2769

2770

2771

2772

2773

2774

2775

2776

2777

2778

2779

2780

2781

2782

2783

2784

2785

2786

2787

2788

2789

2790

2791

2792

2793

2794

2795

2796

2797

2798

2799

2800

2801

2802

2803

2804

2805

2806

2807

2808

2809

2810

2811

2812

2813

2814

2815

2816

2817

2818

2819

2820

2821

2822

2823

2824

2825

2826

2827

2828

parameters:

- name: name

in: path

required: true

schema:

type: string

description: The name of the virtual directory

responses:

'200' :

description: Returning the information of the virtual directory

content:

application/json:

schema:

' 401 ' :

\$ref: '\#/components/schemas/Virtualdirectory'

description: Not authorized

' 404 ' : put:

description: The named virtual directory could not be found

tags:

- Virtual directory

summary: Uploads a virtual directory to the list of virtual directories

description: Uploads a virtual directory to the list of virtual directories

operationId: cloudmesh.virtual directory.add

requestBody:

description: The virtual directory to be uploaded

required: true

content:

application/json:

schema:

responses:

\$ref: '\#/components/schemas/Virtualdirectory'

' 200 ' :

description: Virtual directory updated or created

' 401 ' :

description: Not authorized

' 404 ' : delete:

description: The named virtual directory could not be found

tags:

- Virtual directory

summary: Deletes the named virtual directory

description: Deletes an virtual directory by name

operationId: cloudmesh.virtualdirectory.delete_by_name

parameters:

- name: name

in: path

required: true

schema:

type: string

description: The name of the virtual directory

responses:

'200' :

description: Deletion successful

' 401 ' :

description: Not authorized

'404' :

description: The named virtual directory could not be found /virtualdirectory/\{name $\} /\{$ filename $\}$ :

get:

tags:

- Virtual directory

summary: Returns the specific file of that virtual directory

description: Returns the specific file of that virtual directory

operationId: cloudmesh.virtualdirectory.file.get_by_name

parameters:

- name: name

in: path

required: true 
NIST Big DATA INTEROPERABILITY FRAMEWORK: VOLUME 8, REFERENCE ARCHITECTURE INTERFACES

2829

2830

2831

2832

2833

2834

2835

2836

2837

2838

2839

2840

2841

2842

2843

2844

2845

2846

2847

2848

2849

2850

2851

2852

2853

2854

2855

2856

2857

2858

2859

2860

2861

2862

2863

2864

2865

2866

2867

2868

2869

2870

2871

2872

2873

2874

2875

2876

2877

2878

2879

2880

2881

2882

2883

2884

2885

2886

2887

2888

2889

2890

2891

2892

2893

2894

2895

2896

2897

schema:

type: string

description: The name of the virtual directory

- name: filename

description: The filename

in: path

required: true

schema:

type: string

responses:

'200' :

description: upload successful

content:

application/json:

schema:

'401' :

\$ref: "filestore.yaml\#/components/schemas/File"

description: Not authorized

'404' : put:

description: The named virtual directory or file could not be found

tags:

- Virtual directory

summary: Updates or adds a virtual file in the virtual directory

description: Updates or adds a virtual file in the virtual directory

operationId: cloudmesh.virtualdirectory.file.add

parameters:

- name: name

in: path

required: true

schema:

type: string

description: The name of the virtual directory

- name: filename

description: The filename

in: path

required: true

schema:

type: string

requestBody:

description: The user to be uploaded

required: true

content:

application/json:

schema:

\$ref: "filestore.yaml\#/components/schemas/File"

responses:

' 200 ' :

description: User added successfully

' 401 ' :

'404' :

description: Not authorized

description: The named virtual directory or file could not be found

delete:

tags:

- Virtual directory

summary: Delete an user in the virtual directory

description: Delete an user in the virtual directory

operationId: cloudmesh.virtualdirectory.file.delete

parameters:

- name: name

in: path

required: true

schema:

type: string

description: The name of the virtual directory

- name: filename

description: The filename

in: path 


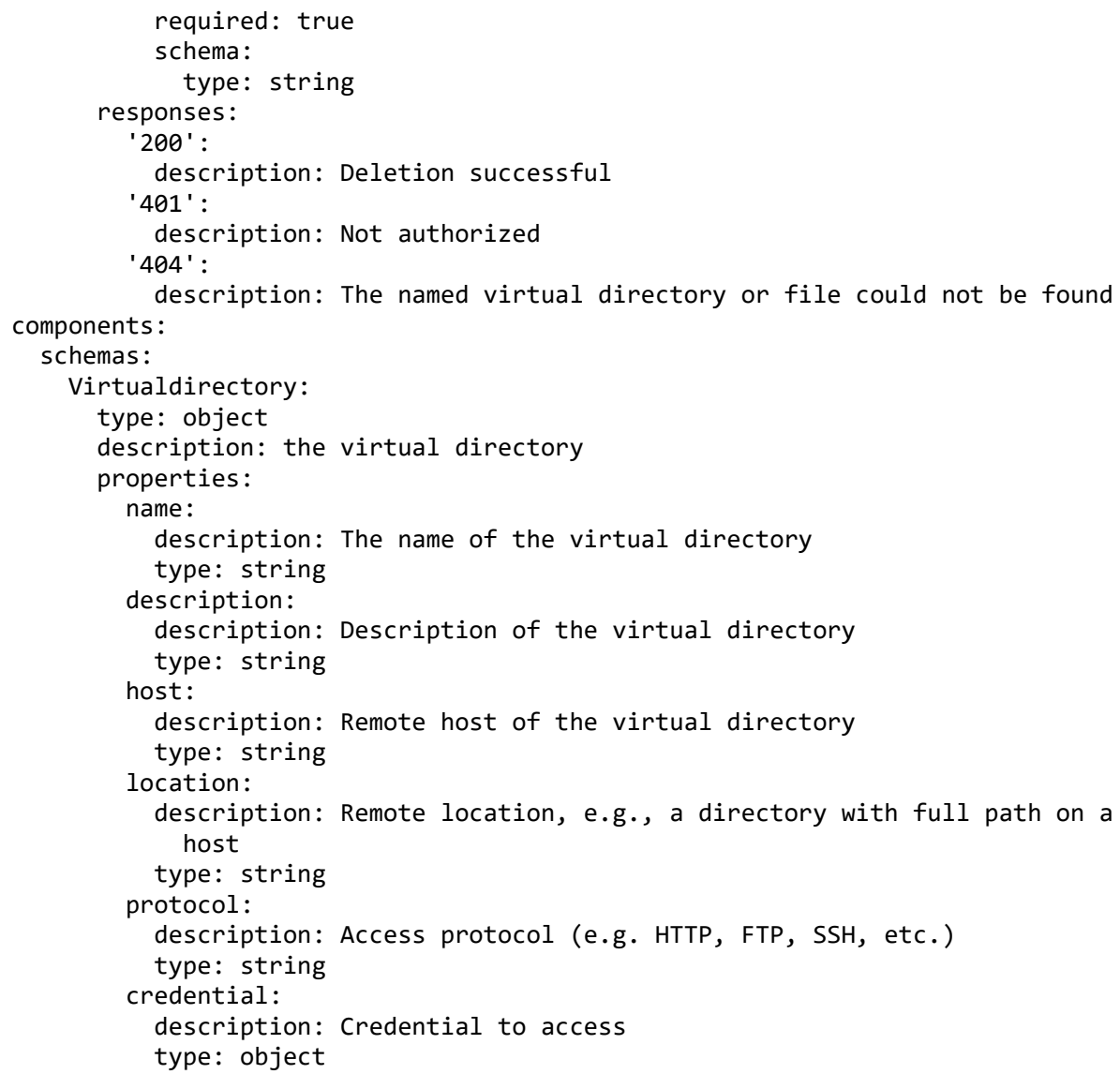

\subsection{COMPUTE MANAGEMENT: VIRTUAL CLUSTERS}

\subsubsection{Virtual Cluster}

A Virtual Cluster is modeled as manager node, and one or more compute nodes. The manager node usually serves as a login node and can be accessed from outside via a public IP. The compute nodes are connected to the manager node via a private, usually high performance (high throughput and low latency), network and, thus, are accessible only from the manager node. To use the virtual cluster, login to the manager node, from which one can login to any compute node, or submit a job to run on the compute nodes.

\subsubsection{Schema Virtualcluster}

\begin{tabular}{|c|c|c|}
\hline Property & Type & Description \\
\hline name & string & The name of the virtual cluster \\
\hline description & string & A description of the virtual cluster \\
\hline owner & string & $\begin{array}{l}\text { Username of the owner of the virtual } \\
\text { cluster }\end{array}$ \\
\hline manager & Node & Manager node of the virtual cluster \\
\hline nodes & array[Node] & List of nodes of the virtual cluster \\
\hline
\end{tabular}




\subsubsection{Schema Node}

\begin{tabular}{lll} 
Property & Type & Description \\
\hline name & string & Name of the node \\
state & string & Power state of the node \\
ncpu & integer & Number of virtual CPUs of the node \\
ram & string & RAM size of the node \\
disk & String & Disk size of the node \\
nics & array[NIC] & List of network interfaces of the node
\end{tabular}

2943 4.8.1.3 Schema NIC

\begin{tabular}{lll} 
Property & Type & Description \\
\hline mac & string & $\begin{array}{l}\text { Media Access Control } \\
\text { (MAC) address of the } \\
\text { node }\end{array}$
\end{tabular}

ip string IP address of the node

2944 4.8.1.4 Paths

\begin{tabular}{lll} 
HTTP & Path & Summary \\
\hline get & $/$ virtualcluster & Returns a list of virtual clusters \\
get & $/$ virtualcluster $/\{$ name $\}$ & Returns the named virtual cluster \\
put & $/$ virtualcluster $/\{$ name $\}$ & Uploads an virtual cluster to the list of virtual clusters \\
delete & $/$ virtualcluster $/\{$ name $\}$ & Deletes the named virtual cluster \\
get & $/$ virtualcluster $/\{$ name $/\{$ node $\}$ & Node of the named virtual cluster \\
put & $/$ virtualcluster $/\{$ name $/\{$ node $\}$ & Updates or adds a node to the virtual cluster \\
delete & $/$ virtualcluster $/\{$ name $/\{$ node $\}$ & Delete a node in the virtual cluster
\end{tabular}

$2945 \quad$ 4.8.1.4.1 /virtualcluster

$2946 \quad$ 4.8.1.4.1.1 GET /virtualcluster

2947 Returns a list of all virtual clusters

2948 Responses

\begin{tabular}{lll} 
Code & Description & Schema \\
\hline 200 & The list of virtual clusters & array[Virtualcluster] \\
401 & Not authorized & String
\end{tabular}

2949 4.8.1.4.2 /virtualcluster/\{name\}

2950 4.8.1.4.2.1 GET/virtualc/uster/\{name\}

2951 Returns a virtual cluster by name 
NIST Big DATA INTEROPERABILITY FRAMEWORK: VOLUME 8, REFERENCE ARCHITECTURE INTERFACES

2952

Responses

Code Description

Schema

200 Returning the information of the virtual cluster Virtualcluster

401 Not authorized String

404 The named virtual cluster could not be found String

2953 Parameters

\begin{tabular}{lllll} 
Name & Located in & Description & Required & Schema \\
\hline name & path & The name of the virtual cluster & True & String
\end{tabular}

2954 4.8.1.4.2.2 PUT /virtualc/uster/\{name\}

2955 Uploads a virtual cluster to the list of virtual clusters

2956 Responses

Code Description Schema

200 Virtual cluster updated or created String

401 Not authorized String

404 The named virtual cluster could not be found String

2957 Request Body

\begin{tabular}{llll} 
Located in & Description & Required & Schema \\
\hline Body & The virtual cluster to be uploaded & True & Virtualcluster
\end{tabular}

\subsection{DELETE /virtualc/uster/\{name\}}

2959 Deletes a virtual cluster by name

2960 Responses

\begin{tabular}{lll} 
Code & Description & Schema \\
\hline 200 & Deletion successful & String \\
401 & Not authorized & String \\
404 & The named virtual cluster could not be found & String
\end{tabular}

2961 Parameters

\begin{tabular}{lllll} 
Name & Located in & Description & Required & Schema \\
\hline name & path & The name of the virtual cluster & True & String
\end{tabular}

2962 4.8.1.4.3 Ivirtualcluster $/$ name\}/\{node\}

2963 4.8.1.4.3.1 GET/virtualc/uster/\{name\}/\{node\}

2964 Returns the specific node of the named virtual cluster. If the node name is manager, the manager node is 2965 used. A compute node cannot be named manager.

2966 Responses 
NIST Big DATA INTEROPERABILITY FRAMEWORK: Volume 8, REFERENCE ARCHITECTURE INTERFACES

\begin{tabular}{lll} 
Code & Description & Schema \\
\hline 200 & Node info & $\underline{\text { Node }}$ \\
401 & Not authorized & String \\
404 & The named virtual cluster or node could not be found & String
\end{tabular}

2967 Parameters

\begin{tabular}{lllll} 
Name & Located in & Description & Required & Schema \\
\hline name & path & The name of the virtual cluster & True & String \\
node & path & The node name & True & String
\end{tabular}

2968 4.8.1.4.3.2 PUT /virtualcluster/\{name\}/\{node\}

2969 Updates or adds a node to the virtual cluster. If the node name is manager, the manager node is uploaded.

2970 Responses

Code Description Schema

$200 \quad$ Node added successfully $\quad$ String

401 Not authorized String

404 The named virtual cluster or node could not be found String

2971 Parameters

\begin{tabular}{lllll} 
Name & Located in & Description & Required & Schema \\
\hline name & path & The name of the virtual cluster & True & String \\
node & path & The node name & True & String
\end{tabular}

2972 Request Body

\begin{tabular}{llll} 
Located in & Description & Required & Schema \\
\hline Body & The node to be uploaded & True & Node
\end{tabular}

2973 4.8.1.4.3.3 DELETE /virtualc/uster/\{name\}/\{node\}

2974 Delete a node in the virtual cluster

2975 Responses

\begin{tabular}{lll} 
Code & Description & Schema \\
\hline 200 & Deletion successful & String \\
401 & Not authorized & String \\
404 & The named virtual cluster or node could not be found & String
\end{tabular}

2976 Parameters

\begin{tabular}{lllll} 
Name & Located in & Description & Required & Schema \\
\hline name & path & The name of the virtual cluster & True & String \\
node & path & The node name & True & String
\end{tabular}




\subsubsection{5 virtualcluster.yaml}

2978

2979

2980

2981

2982

2983

2984

2985

2986

- 2987

2988

2989

2990

2991

2992

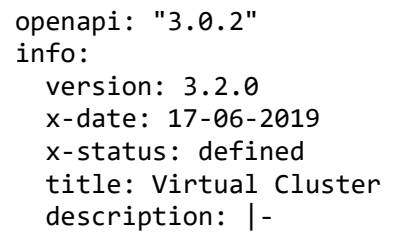


NIST Big DATA INTEROPERABILITY FRAMEWORK: VOLUME 8, REFERENCE ARCHITECTURE INTERFACES

3045

3046

3047

3048

3049

3050

3051

3052

3053

3054

3055

3056

3057

3058

3059

3060

3061

3062

3063

3064

3065

3066

3067

3068

3069

3070

3071

3072

3073

3074

3075

3076

3077

3078

3079

3080

3081

3082

3083

3084

3085

3086

3087

3088

3089

3090

3091

3092

3093

3094

3095

3096

3097

3098

3099

3100

3101

3102

3103

3104

3105

3106

3107

3108

3109

3110

3111

3112

3113

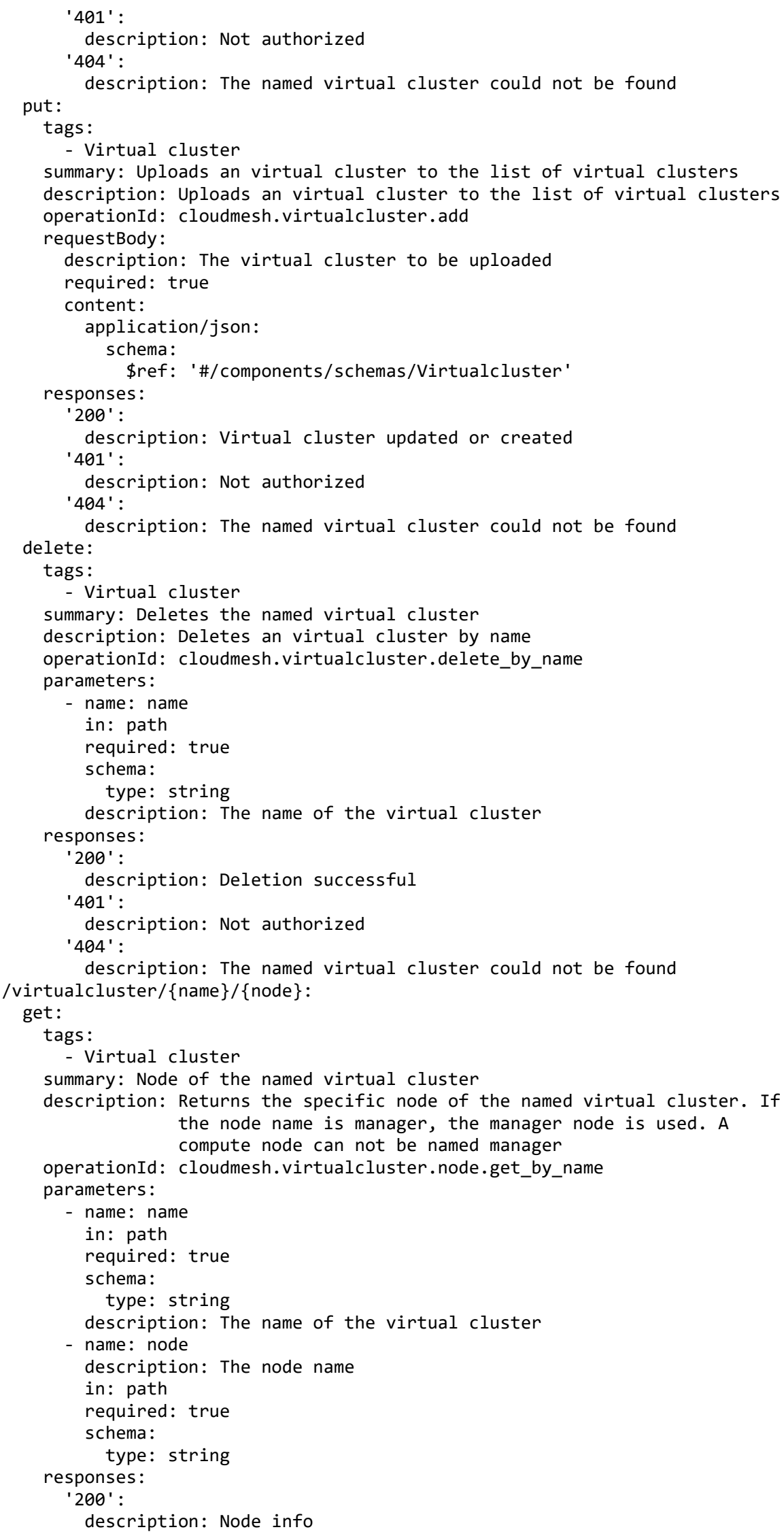


NIST Big DATA INTEROPERABILITY FRAMEWORK: Volume 8, REFERENCE ARCHITECTURE INTERFACES

3114

3115

3116

3117

3118

3119

3120

3121

3122

3123

3124

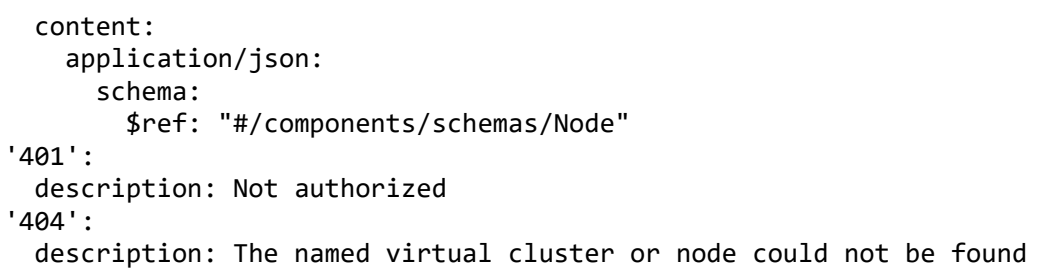


3183

3184

3185

3186

3187

3188

3189

3190

3191

3192

3193

- 3194

3195

3196

3197

3198

3199

3200

3201

3202

3203

3204

3205

3206

3207

3208

3209

3210

3211

3212

3213

3214

3215

3216

3217

3218

3219

3220

3221

3222

3223

3224

3225

3226

3227

3228

3229

3230

3231

3232

3233

3234

3235

3236

3237

3238

3239

3240

3241

3242

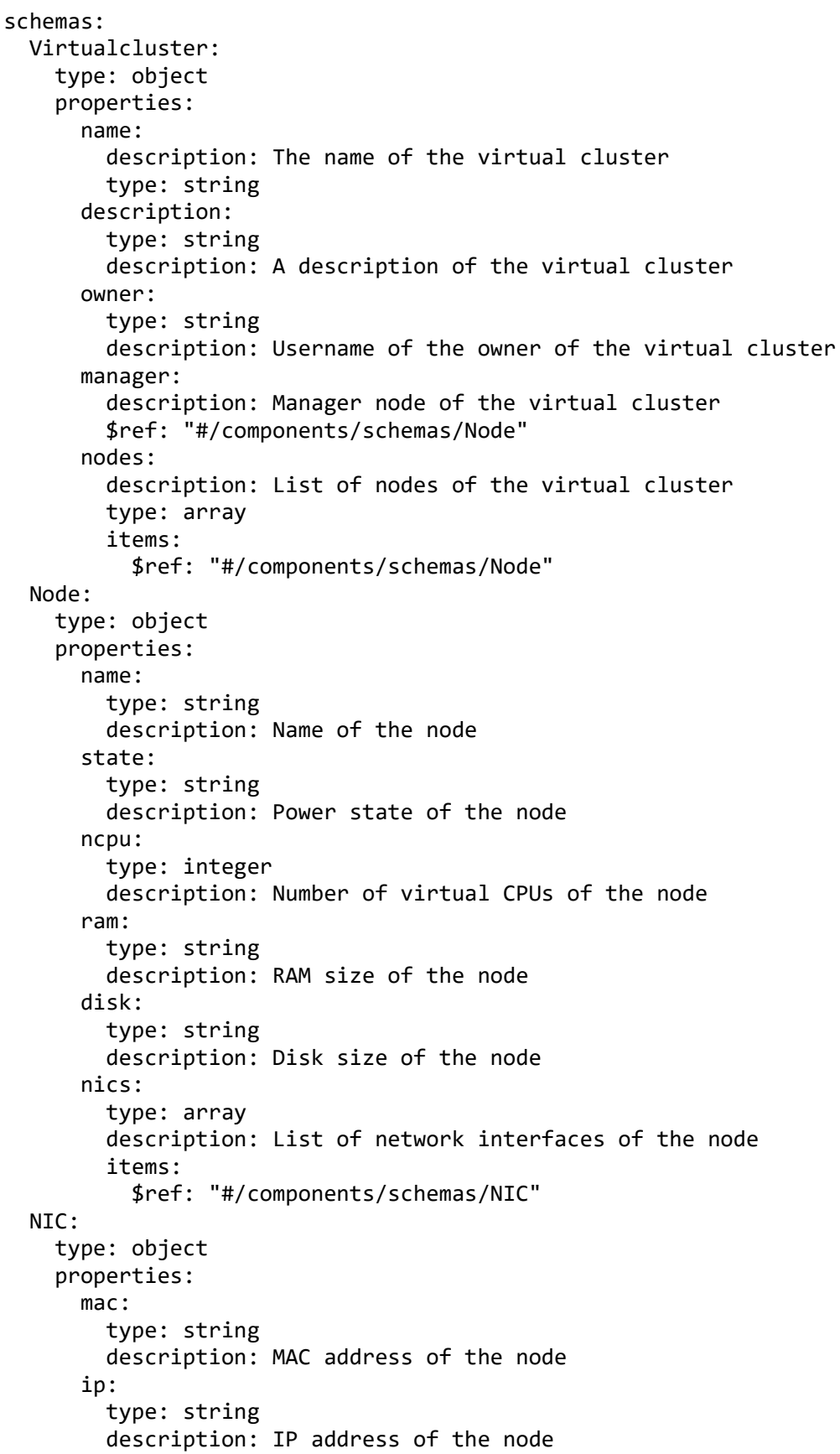

\subsubsection{NETWORK OF NODES}

A Network of Nodes (NON) contains a number of compute nodes that are connected by a network and can be reached from the compute nodes. The concept is a generalization of the term Network of Workstations. In contrast to a Virtual Cluster, it does not have a dedicated manager node. Network of nodes can be real or virtual. The same security context can be used to authenticate to all nodes in the network of nodes. This is typically done with a public key store in which all keys are stored that allow access to the nodes. 


\subsubsection{Schema Non}

\begin{tabular}{lll} 
Property & Type & Description \\
\hline name & string & The name of the network of nodes \\
description & string & A description of the network of nodes \\
nodes & array[Node] & List of nodes of the network of nodes
\end{tabular}

\section{4.8.2.2 Paths}

\begin{tabular}{lll} 
HTTP & Path & Summary \\
\hline get & $/$ non & Returns a list of network of nodes \\
put & $/$ non $/\{$ name $\}$ & Uploads a network of nodes to the list of network of nodes \\
get & $/$ non $/\{$ name $\} /$ publickeystore & Returns the information of the key store \\
delete & $/$ non $/\{$ name $\} /$ publickeystore & Deletes the key store \\
put & $/$ non $/\{$ name $/$ publickeystore & Adds a key store \\
get & $/$ non $/\{$ name $/$ node & Returns the named network of nodes \\
delete & $/$ non $/\{$ name $\} /$ node & Deletes the named network of nodes \\
get & $/$ non $/\{$ name $\} /$ node $/$ nnode $\}$ & Node of the named network of nodes \\
put & $/$ non $/\{$ name $/$ node $/$ nnode $\}$ & Updates or adds a node to the network of nodes \\
delete & $/$ non $/\{$ name $/$ node $/$ nnode $\}$ & Delete a node in the network of nodes
\end{tabular}

$3245 \quad$ 4.8.2.2.1 Inon

3246 4.8.2.2.1.1 GET/non

3247 Returns a list of all network of nodes

3248 Responses

\begin{tabular}{lll} 
Code & Description & Schema \\
\hline 200 & The list of network of nodes & array[Non] \\
401 & Not authorized & String
\end{tabular}

3249 4.8.2.2.2 Inon/\{name\}

3250 4.8.2.2.2.1 PUT /non/\{name\}

3251 Uploads a network of nodes to the list of network of nodes

3252 Responses

\begin{tabular}{lll} 
Code & Description & Schema \\
\hline 200 & Network of nodes updated or created & String \\
400 & Error updating network of nodes & String \\
401 & Not authorized & String
\end{tabular}

3253 Request Body 
NIST Big DATA INTEROPERABILITY FRAMEWORK: VOLUME 8, REFERENCE ARCHITECTURE INTERFACES

\begin{tabular}{llll} 
Located in & Description & Required & Schema \\
\hline Body & The network of nodes to be uploaded & True & Non
\end{tabular}

\subsection{Inon/\{name\}/publickeystore}

\section{4.8.2.2.3.1 GET/non/\{name\}/publickeystore}

3256 Returns a network of nodes by name

3257 Responses

Code Description Schema

200 Returning the information of the network of nodes string

401 Not authorized String

3258 Parameters

\begin{tabular}{lllll} 
Name & Located in & Description & Required & Schema \\
\hline name & path & The name of the network of nodes & True & String
\end{tabular}

3259 4.8.2.2.3.2 DELETE /non/\{name\}/publickeystore

3260 Deletes a network of nodes by name

3261 Responses

\begin{tabular}{lll} 
Code & Description & Schema \\
\hline 200 & Deletion successful & String \\
401 & Not authorized & String
\end{tabular}

3262 Parameters

\begin{tabular}{lllll} 
Name & Located in & Description & Required & Schema \\
\hline name & path & The name of the network of nodes & True & String
\end{tabular}

3263 4.8.2.2.3.3 PUT /non/\{name\}/publickeystore

3264 Updates or adds a node to the network of nodes.

3265 Responses

\begin{tabular}{lll} 
Code & Description & Schema \\
\hline 200 & Node keystore added successfully & String \\
401 & Not authorized & String
\end{tabular}

3266 Parameters

\begin{tabular}{lllll} 
Name & Located in & Description & Required & Schema \\
\hline name & path & The name of the network of nodes & True & String
\end{tabular}




\subsection{Inon/\{name\}/node}

\subsection{GET /non/\{name\}/node}

3269 Returns a network of nodes by name

3270 Responses

\begin{tabular}{lll} 
Code & Description & Schema \\
\hline 200 & Returning the information of the node & $\underline{\text { Non }}$ \\
401 & Not authorized & String \\
404 & The named network of nodes could not be found & String
\end{tabular}

3271 Parameters

\begin{tabular}{lllll} 
Name & Located in & Description & Required & Schema \\
\hline name & path & The name of the network of nodes & True & String
\end{tabular}

3272 4.8.2.2.4.2 DELETE /non/\{name\}/node

3273 Deletes a network of nodes by name

3274 Responses

\begin{tabular}{lll} 
Code & Description & Schema \\
\hline 200 & Deletion successful & String \\
401 & Not authorized & String \\
404 & The named network of nodes could not be found & String
\end{tabular}

3275 Parameters

\begin{tabular}{lllll} 
Name & Located in & Description & Required & Schema \\
\hline name & path & The name of the network of nodes & True & String
\end{tabular}

$3276 \quad$ 4.8.2.2.5 Inon/\{name\}/node/ node\}

3277 4.8.2.2.5.1 GET /non/\{name\}/node/\{node\}

3278 Returns the specific node of the named network of nodes.

3279 Responses

\begin{tabular}{lll} 
Code & Description & Schema \\
\hline 200 & Node info & $\underline{\text { Node }}$ \\
401 & Not authorized & String
\end{tabular}

$3280 \quad$ Parameters

\begin{tabular}{lllll} 
Name & Located in & Description & Required & Schema \\
\hline name & path & The name of the network of nodes & True & String \\
node & path & The node name & True & String
\end{tabular}




\subsection{PUT /non/\{name\}/node/\{node\}}

3282 Updates or adds a node to the network of nodes

3283 Responses

Code Description Schema

200 Node added successfully String

401 Not authorized String

3284 Parameters

Name Located in Description Required Schema

name path The name of the network of nodes True String

node path The node name True String

3285 Request Body

Located in Description Required Schema

Body The node to be uploaded True Node

\subsection{DELETE /non/\{name\}/node/\{node\}}

3287 Delete a node in the network of nodes

3288 Responses

Code Description Schema

200 Deletion successful String

401 Not authorized String

3289 Parameters

\begin{tabular}{lllll} 
Name & Located in & Description & Required & Schema \\
\hline name & path & The name of the network of nodes & True & String \\
node & path & The node name & True & String
\end{tabular}

\subsubsection{3 non.yaml}

openapi: "3.0.2"

info:

version: 3.2 .0

$x$-date: 17-06-2019

$\mathrm{x}$-status: defined

title: Network of Nodes

description: |-

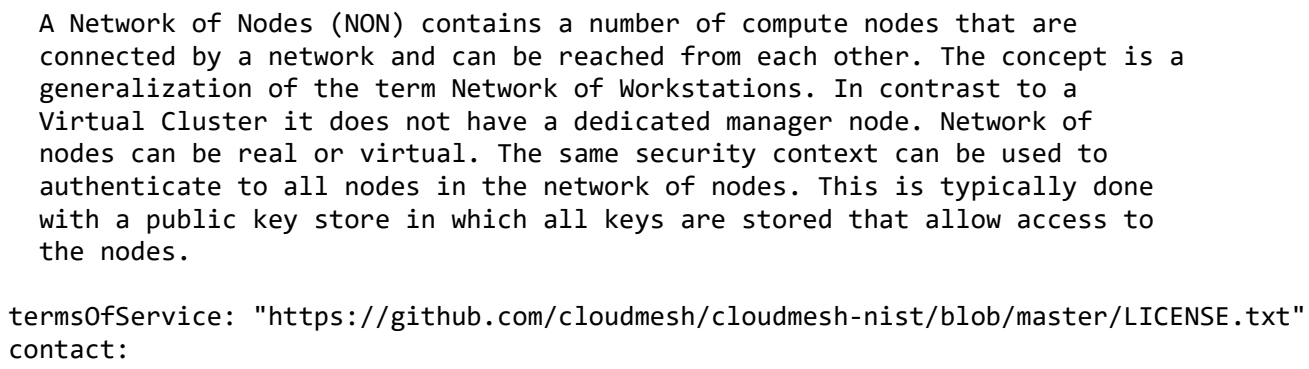


NIST Big DATA INTEROPERABILITY FRAMEWORK: Volume 8, REFERENCE ARCHITECTURE INTERFACES

name: NIST BDRA Interface Subgroup Service

url: https://cloudmesh-community.github.io/nist/spec/

license:

name: Apache 2.0

url: https://github.com/cloudmesh/cloudmesh-nist/blob/master/LICENSE.txt servers:

- url: /cloudmesh/v3

paths:

/non:

get :

tags:

- Network of nodes

summary: Returns a list of network of nodes

description: Returns a list of all network of nodes

operationId: cloudmesh.non.list

responses:

'200' :

description: The list of network of nodes

content:

application/json:

schema:

type: array

items:

'401' :

\$ref: '\#/components/schemas/Non'

description: Not authorized

/non/ $\{$ name $\}$ :

put:

tags:

- Network of nodes

summary: Uploads a network of nodes to the list of network of nodes

description: Uploads a network of nodes to the list of network of nodes

operationId: cloudmesh.non.add

requestBody:

description: The network of nodes to be uploaded

required: true

content:

application/json:

schema:

\$ref: '\#/components/schemas/Non'

responses:

'200':

description: Network of nodes updated or created.

'400' :

description: Error updating network of nodes

' 401 ' :

description: Not authorized

/non/ \{name\}/publickeystore:

get:

tags:

- Non

summary: Returns the information of the key store

description: Returns a network of nodes by name

operationId: cloudmesh.non.keystore.find_by_name

parameters:

- name: name

in: path

required: true

schema:

type: string

description: The name of the network of nodes

responses:

'200' :

description: Returning the information of the network of nodes

content:

application/json:

schema:

type: string

description: the endpoint of the publickeystore 
NIST Big DATA INTEROPERABILITY FRAMEWORK: VOLUME 8, REFERENCE ARCHITECTURE INTERFACES

3379

3380

3381

3382

3383

3384

3385

3386

3387

3388

3389

3390

3391

3392

3393

3394

3395

3396

3397

3398

3399

3400

3401

3402

3403

3404

3405

3406

3407

3408

3409

3410

3411

3412

3413

3414

3415

3416

3417

3418

3419

3420

3421

3422

3423

3424

3425

3426

3427

3428

3429

3430

3431

3432

3433

3434

3435

3436

3437

3438

3439

3440

3441

3442

3443

3444

3445

3446

3447

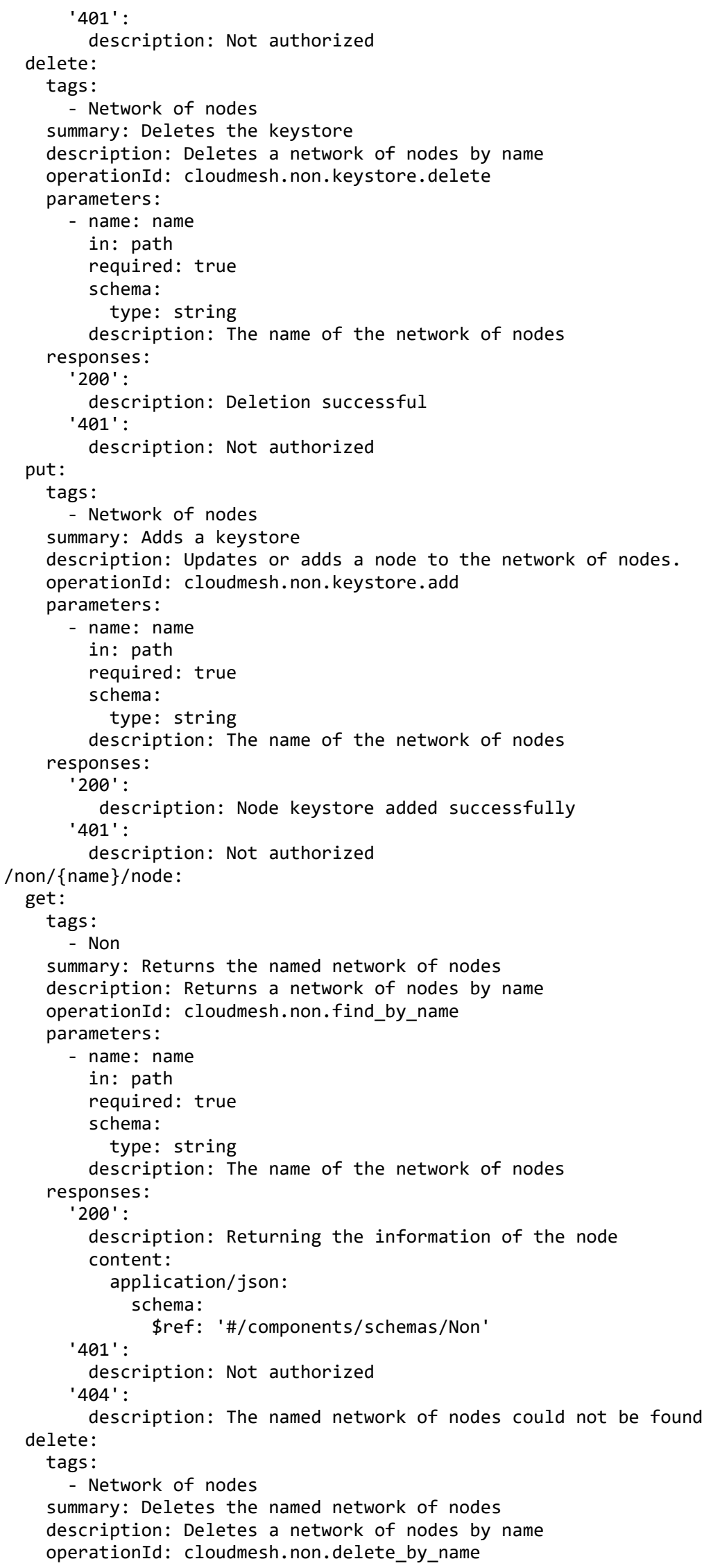


NIST Big DATA INTEROPERABILITY FRAMEWORK: VOLUME 8, REFERENCE ARCHITECTURE INTERFACES

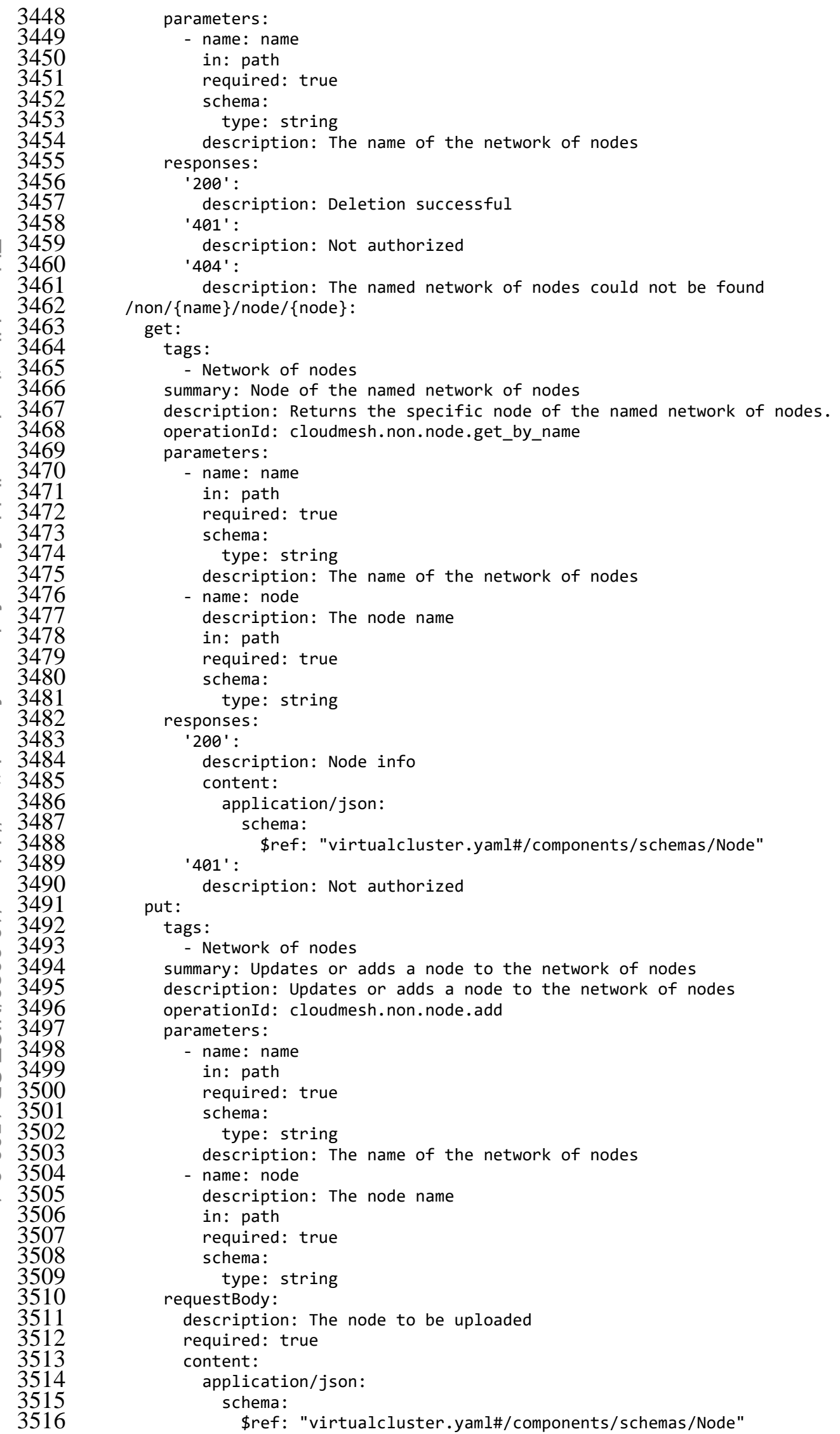


NIST Big DATA INTEROPERABILITY FRAMEWORK: VOLUME 8, REFERENCE ARCHITECTURE INTERFACES

3517

3518

3519

3520

3521

3522

3523

3524

3525

3526

3527

거 3528

3529

3530

3531

3532

3533

3534

3535

3536

3537

3538

3539

3540

3541

3542

3543

3544

3545

3546

3547

3548

3549

3550

3551

3552

3553

3554

3555

3556

3557

3558

3559

3560

3561

3562

3563

3564

3565

3566

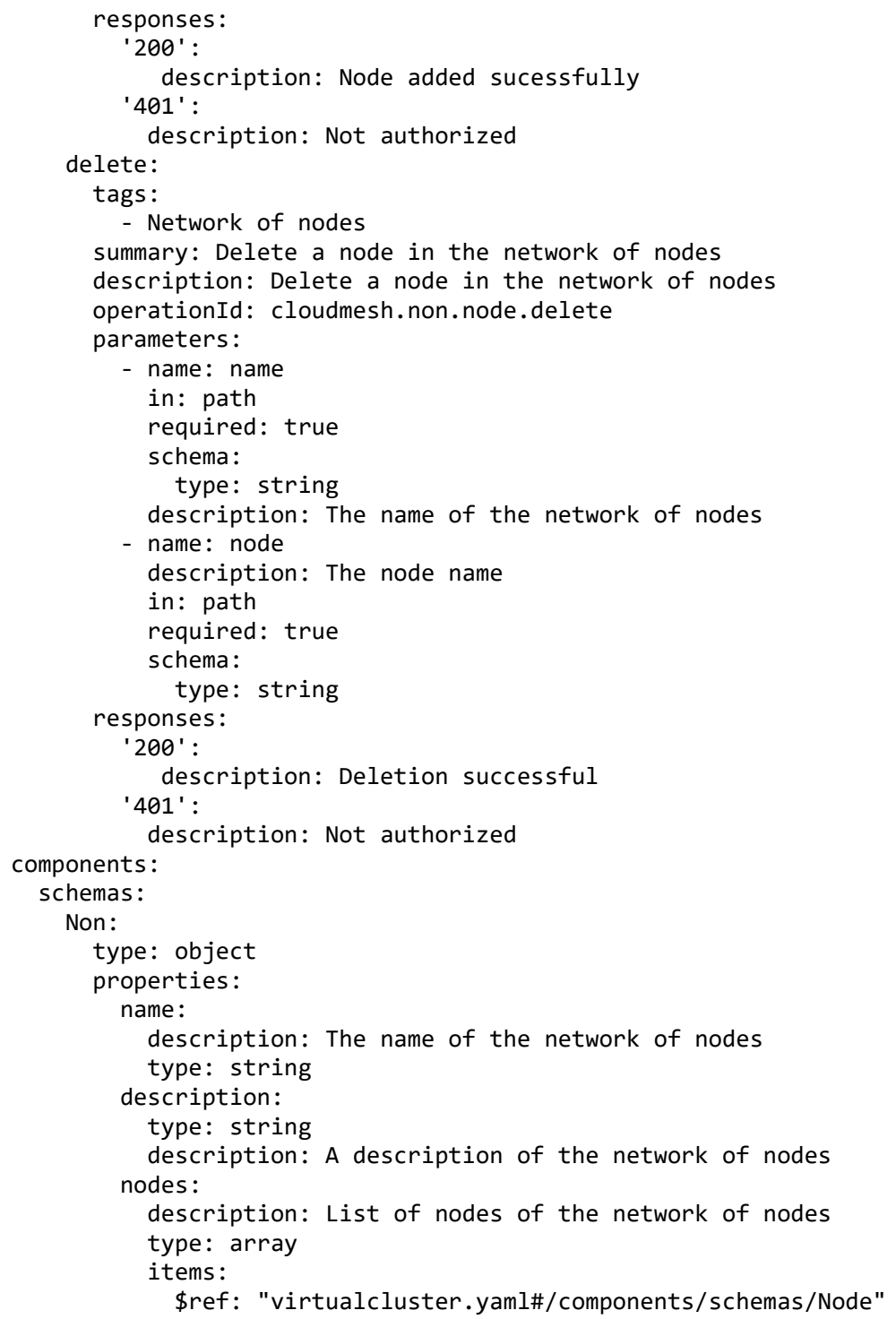

\subsubsection{SCHEDULER}

A scheduler allows for control of the execution of tasks based on a policy. Schedulers may allow the assignment of different policies to define the order of the tasks. A scheduler returns the next task to be executed. Tasks can be added and deleted.

\subsubsection{Schema Task}

\begin{tabular}{lll} 
Property & Type & Description \\
\hline Name & string & Name of the scheduler \\
User & string & The username the task belongs to \\
description & string & The description of the task \\
Kind & string & The kind of the task
\end{tabular}




\subsubsection{Schema Policy}

\begin{tabular}{lrl} 
Property & Type & Description \\
\hline Name & string & Name of the scheduler policy \\
description & string & The description of the policy \\
Kind & string & The kind of the policy \\
parameters & string & Parameters to define the behavior of the scheduler
\end{tabular}

\section{4.8.3.3 Paths}

\begin{tabular}{lll} 
HTTP & Path & Summary \\
\hline get & /task & Returns a list of tasks \\
get & $/$ task $/\{$ name\} & Returns the named task \\
put & $/$ task $/\{$ name\} & Uploads a task to the list of tasks \\
delete & $/$ task/\{name\} & Deletes the named task \\
get & /policy & Returns the policy found \\
put & /policy & Uploads the policy
\end{tabular}

3569 4.8.3.3.1 Itask

3570 4.8.3.3.1.1 GET/task

3571 Returns a list of all tasks

3572 Responses

\begin{tabular}{lll} 
Code & Description & Schema \\
\hline 200 & The list of tasks & Array[Task] \\
401 & Not authorized & String
\end{tabular}

3573 4.8.3.3.2 Itask/\{name\}

3574 4.8.3.3.2.1 GET/task/\{name\}

3575 Returns a task by name

3576 Responses

\begin{tabular}{lll} 
Code & Description & Schema \\
\hline 200 & Returning the information of the task & $\underline{\text { Task }}$ \\
401 & Not authorized & String \\
404 & The named task could not be found & String
\end{tabular}


NIST Big DATA INTEROPERABILITY FRAMEWORK: VOLUME 8, REFERENCE ARCHITECTURE INTERFACES

Parameters

\begin{tabular}{lllll} 
Name & Located in & Description & Required & Schema \\
\hline name & path & The name of the task & True & String \\
operation & query & Show the task but do not remove it from the queue & False & String
\end{tabular}

\subsection{PUT/task/\{name\}}

3579 Uploads a task to the list of tasks

3580 Responses

\begin{tabular}{lll} 
Code & Description & Schema \\
\hline 200 & Task updated & String \\
401 & Not authorized & String
\end{tabular}

3581 Parameters

\begin{tabular}{lllll} 
Name & Located in & Description & Required & Schema \\
\hline name & path & The name of the task & True & String
\end{tabular}

3582 Request Body

\begin{tabular}{llll} 
Located in & Description & Required & Schema \\
\hline Body & The task to be uploaded & True & $\underline{\text { Task }}$
\end{tabular}

\subsection{DELETE /task/\{name\}}

3584 Deletes a task by name

3585 Responses

\begin{tabular}{lll} 
Code & Description & Schema \\
\hline 200 & Deletion successful & String \\
400 & Error to delete the task & String \\
401 & Not authorized & String \\
404 & The named task could not be found & String
\end{tabular}

3586 Parameters

\begin{tabular}{lllll} 
Name & Located in & Description & Required & Schema \\
\hline name & path & The name of the task & True & String
\end{tabular}

\section{4.8.3.3.3 /policy}

3588 4.8.3.3.3.1 GET/policy

3589 Returns the policy 
NIST Big DATA INTEROPERABILITY FRAMEWORK: VOLUME 8, REFERENCE ARCHITECTURE INTERFACES

Responses

Code Description Schema

200 The policy array[Policy]

401 Not authorized String

3591

\subsection{PUT /policy}

Uploads a task to the list of tasks

Responses

Code Description Schema

200 Task updated String

400 Error updating String

401 Not authorized String

Request Body

Located in Description Required Schema

Body The policy to be uploaded True $\underline{\text { Policy }}$

\subsubsection{4 queue.yaml}

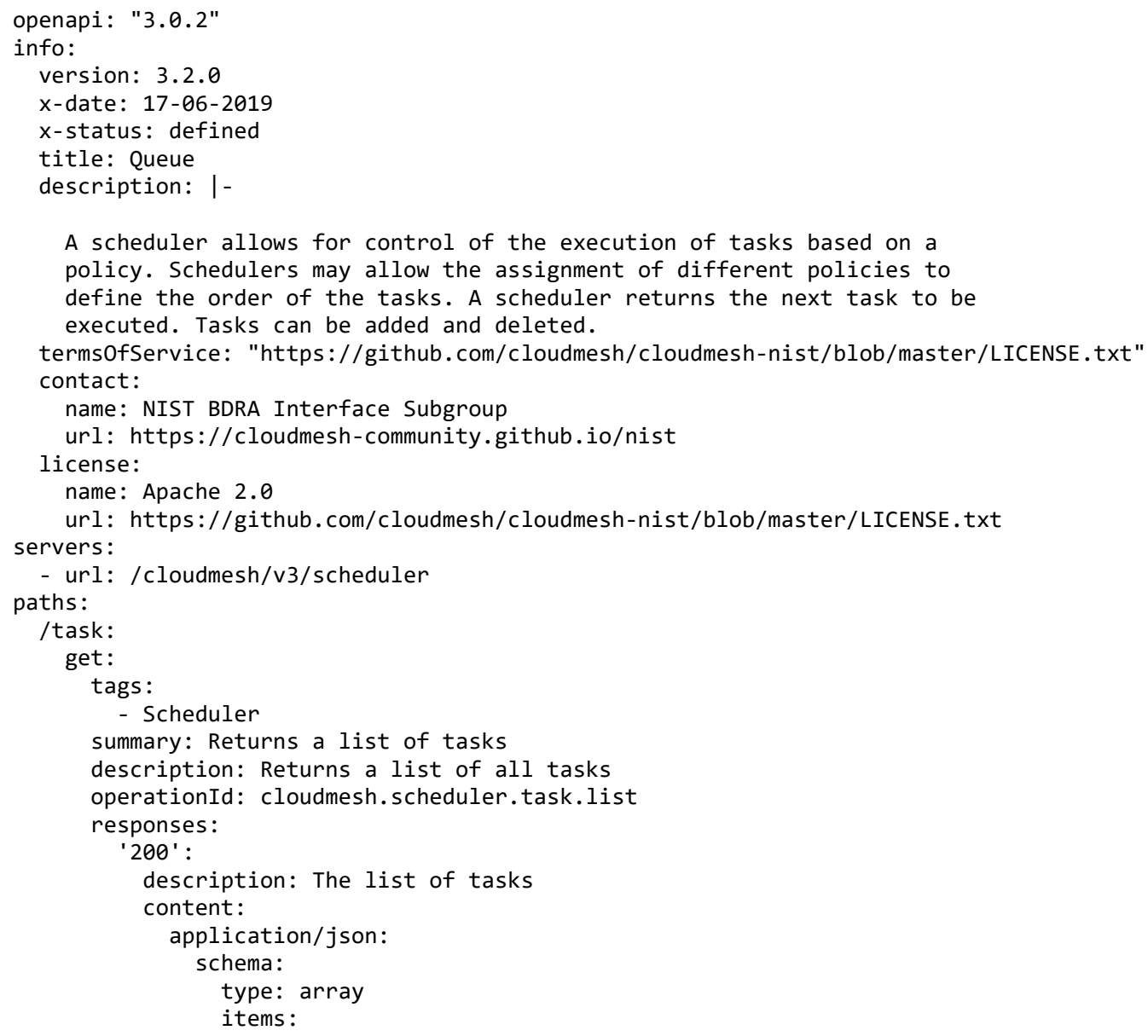


NIST Big DATA INTEROPERABILITY FRAMEWORK: Volume 8, REFERENCE ARCHITECTURE INTERFACES

3633

3634

3635

3636

3637

3638

3639

3640

3641

3642

3643

3644

3645

3646

3647

3648

3649

3650

3651

3652

3653

3654

3655

3656

3657

3658

3659

3660

3661

3662

3663

3664

3665

3666

3667

3668

3669

3670

3671

3672

3673

3674

3675

3676

3677

3678

3679

3680

3681

3682

3683

3684

3685

3686

3687

3688

3689

3690

3691

3692

3693

3694

3695

3696

3697

3698

3699

3700

3701

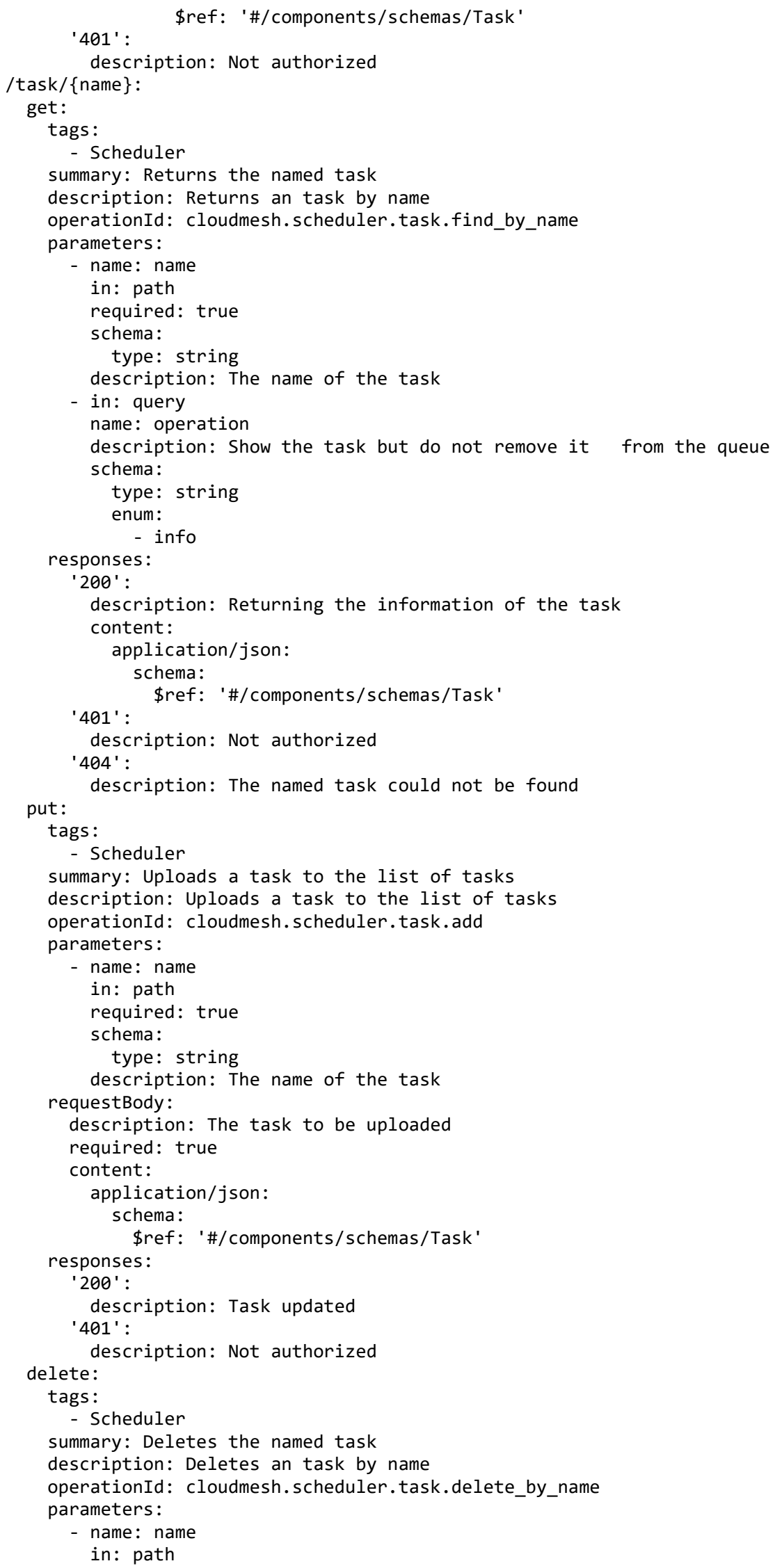


NIST Big DATA INTEROPERABILITY FRAMEWORK: VOLUME 8, REFERENCE ARCHITECTURE INTERFACES

3702

3703

3704

3705

3706

3707

3708

3709

3710

3711

3712

3713

3714

3715

3716

3717

3718

3719

3720

3721

3722

3723

3724

3725

3726

3727

3728

3729

3730

3731

3732

3733

3734

3735

3736

3737

3738

3739

3740

3741

3742

3743

3744

3745

3746

3747

3748

3749

3750

3751

3752

3753

3754

3755

3756

3757

3758

3759

3760

3761

3762

3763

3764

3765

3766

3767

3768

3769

3770

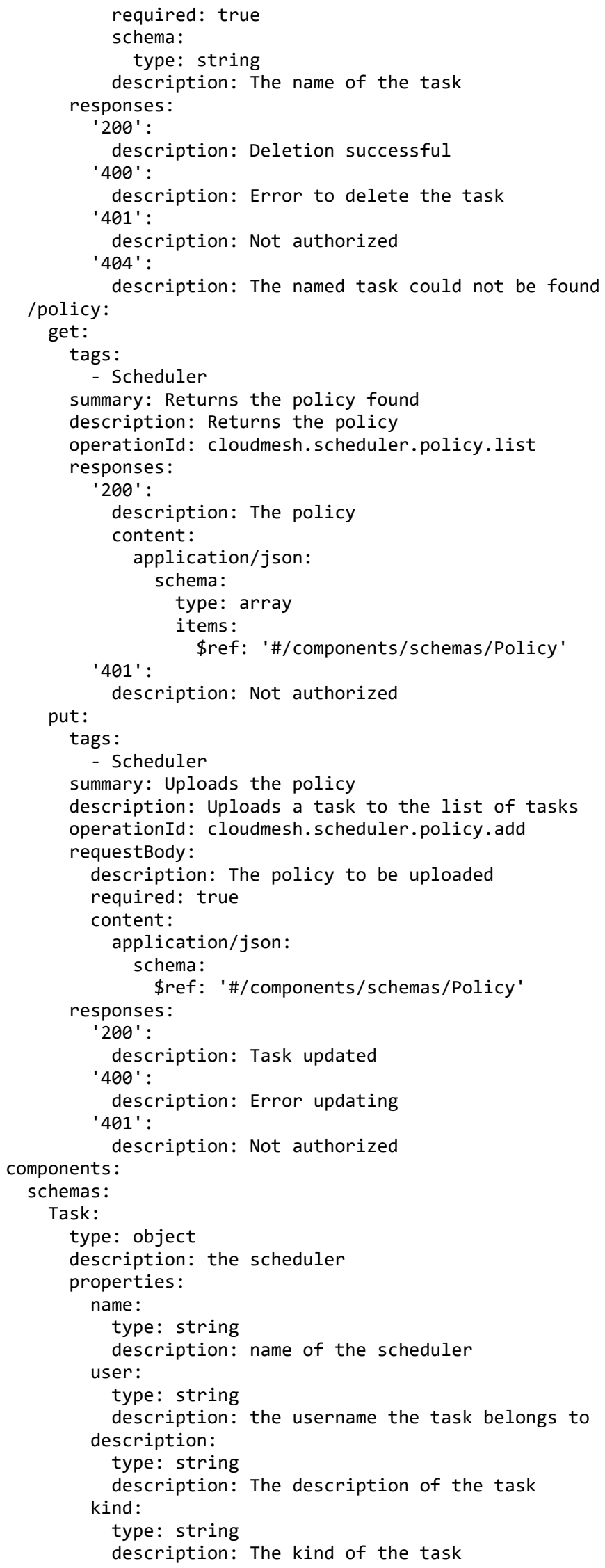




\subsubsection{Schema Policy}

Property Type Description

Name string Name of the scheduler policy

Description string The description of the policy

Kind string The kind of the policy

Parameters string Parameters to define the behavior of the scheduler

3793 4.8.4.3 Paths

\begin{tabular}{lll} 
HTTP & Path & Summary \\
\hline get & /task & Returns a list of tasks \\
get & $/$ task $/$ name $\}$ & Returns the named task \\
put & $/$ task $/\{$ name $\}$ & Uploads a task to the list of tasks \\
delete & $/$ task $/\{$ name $\}$ & Deletes the named task \\
get & $/$ policy & Returns the policy \\
put & /policy & Uploads the policy
\end{tabular}


4.8.4.3.1 Itask

3795

\subsection{GET/task}

3796

Returns a list of all tasks

3797 Responses

\begin{tabular}{lll} 
Code & Description & Schema \\
\hline 200 & The list of tasks & Array[Task] \\
400 & No tasks found & String \\
401 & Not authorized & String
\end{tabular}

$3798 \quad$ 4.8.4.3.2 Itask/\{name $\}$

\subsection{GET/task/\{name\}}

3800 Returns a task by name

3801 Responses

\begin{tabular}{lll} 
Code & Description & Schema \\
\hline 200 & Returning the information of the task & $\underline{\text { Task }}$ \\
400 & No task found & String \\
401 & Not authorized & String \\
404 & The named task could not be found & String
\end{tabular}

Parameters

\begin{tabular}{lllll} 
Name & Located in & Description & Required & Schema \\
\hline Name & path & The name of the task & True & String \\
operation & query & ERROR: description missing & False & String
\end{tabular}

3803 4.8.4.3.2.2 PUT/task/\{name\}

3804 Uploads a task to the list of tasks

3805 Responses

\begin{tabular}{lll} 
Code & Description & Schema \\
\hline 200 & Task updated & String \\
400 & Error updating task. & String \\
401 & Not authorized & String
\end{tabular}

3806 Parameters

\begin{tabular}{lllll} 
Name & Located in & Description & Required & Schema \\
\hline name & path & The name of the task & True & String
\end{tabular}


NIST Big DATA INTEROPERABILITY FRAMEWORK: VOLUME 8, REFERENCE ARCHITECTURE INTERFACES

3807

Request Body

\begin{tabular}{llll} 
Located in & Description & Required & Schema \\
\hline Body & The task to be uploaded & True & $\underline{\text { Task }}$
\end{tabular}

3808

\subsection{DELETE /task/\{name\}}

3809 Deletes a task by name

3810 Responses

\begin{tabular}{lll} 
Code & Description & Schema \\
\hline 200 & Deletion successful & String \\
400 & No task found & String \\
401 & Not authorized & String \\
404 & The named task could not be found & String
\end{tabular}

3811 Parameters

\begin{tabular}{lllll} 
Name & Located in & Description & Required & Schema \\
\hline name & path & The name of the task & True & String
\end{tabular}

$3812 \quad$ 4.8.4.3.3 Ipolicy

3813 4.8.4.3.3.1 GET/policy

3814 Returns the policy

3815 Responses

\begin{tabular}{lll} 
Code & Description & Schema \\
\hline 200 & The policy & array[Policy] \\
400 & No tasks found & String \\
401 & Not authorized & String
\end{tabular}

3816 4.8.4.3.3.2 PUT /policy

3817 Uploads a task to the list of tasks

3818 Responses

\begin{tabular}{lll} 
Code & Description & Schema \\
\hline 200 & Task updated & String \\
400 & Error updating task & String \\
401 & Not authorized & String
\end{tabular}

3819 Request Body

Located in Description Required Schema

Body The policy True




\subsubsection{4 scheduler.yaml}

3821

3822

3823

3824

3825

3826

3827

3828

3829

3830

3831

3832

3833

3834

3835

3836

3837

3838

3839

3840

3841

3842

3843

3844

3845

3846

3847

3848

3849

3850

3851

3852

3853

3854

3855

3856

3857

3858

3859

3860

3861

3862

3863

3864

3865

3866

3867

3868

3869

3870

3871

3872

3873

3874

3875

3876

3877

3878

3879

3880

3881

3882

3883

3884

3885

3886

3887

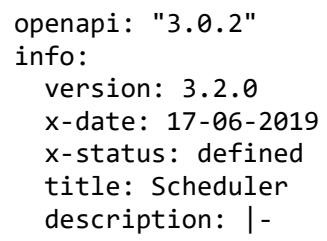


NIST Big DATA INTERoperability FrameWORK: Volume 8, REFERENCE ARCHITECTURE INTERFACES

3888

3889

3890

3891

3892

3893

3894

3895

3896

3897

3898

3899

3900

3901

3902

3903

3904

3905

3906

3907

3908

3909

3910

3911

3912

3913

3914

3915

3916

3917

3918

3919

3920

3921

3922

3923

3924

3925

3926

3927

3928

3929

3930

3931

3932

3933

3934

3935

3936

3937

3938

3939

3940

3941

3942

3943

3944

3945

3946

3947

3948

3949

3950

3951

3952

3953

3954

3955

3956

description: Returning the information of the task

content:

application/json:

schema:

\$ref: '\#/components/schemas/Task'

' 400 ' :

description: No task found

' 401 ' :

description: Not authorized

'404' :

description: The named task could not be found

put:

tags:

- Task

summary: Uploads a task to the list of tasks

description: Uploads a task to the list of tasks

operationId: cloudmesh.task.add

parameters:

- name: name

in: path

required: true

schema:

type: string

description: The name of the task

requestBody:

description: The task to be uploaded

required: true

content:

application/json:

schema:

\$ref: '\#/components/schemas/Task'

responses:

'200' :

description: Task updated

'400' :

description: Error updating task.

' 401 ' :

description: Not authorized

delete:

tags:

- Task

summary: Deletes the named task

description: Deletes an task by name

operationId: cloudmesh.task.delete_by_name

parameters:

- name: name

in: path

required: true

schema:

type: string

description: The name of the task

responses:

' 200 ' :

description: Deletion successful

'400' :

description: No task found

' 401 ' :

description: Not authorized

'404' :

description: The named task could not be found

/policy:

get:

tags:

- Task

summary: Returns the policy

description: Returns the policy

operationId: cloudmesh.task.policy.list

responses:

'200' : 
NIST Big DATA INTEROPERABILITY FRAMEWORK: VOLUME 8, REFERENCE ARCHITECTURE INTERFACES

3957

3958

3959

3960

3961

3962

3963

3964

3965

3966

3967

3968

3969

3970

3971

3972

3973

3974

3975

3976

3977

3978

3979

3980

3981

3982

3983

3984

3985

3986

3987

3988

3989

3990

3991

3992

3993

3994

3995

3996

3997

3998

3999

4000

4001

4002

4003

4004

4005

4006

4007

4008

4009

4010

4011

4012

4013

4014

4015

4016

4017

4018

4019

4020

4021

4022

4023

4024

description: The policy

content:

application/json:

schema:

type: array

items:

\$ref: '\#/components/schemas/Policy'

'400' :

description: No tasks found

' 401 ' :

description: Not authorized

put:

tags:

- Task

summary: Uploads the policy

description: Uploads a task to the list of tasks

operationId: cloudmesh.task.policy.add

requestBody:

description: The Policy

required: true

content:

application/json:

schema:

\$ref: '\#/components/schemas/Policy'

responses:

'200':

description: Task updated

'400' :

description: Error updating task

'401' :

description: Not authorized

components:

schemas:

Task:

type: object

description: The scheduler

properties:

name:

type: string

description: Name of the scheduler

user:

type: string

description: The username the task belongs to

description:

type: string

description: The description of the task

kind:

type: string

description: The kind of the task

content:

type: string

Policy:

description: The content of the task

type: object

description: The policy of the scheduler

properties:

name:

type: string

description: Name of the scheduler policy

description:

type: string

description: The description of the policy

kind:

type: string

description: The kind of the policy

parameters:

type: string

description: parameters to define the behavior of the scheduler 


\subsection{COMPUTE MANAGEMENT: VIRTUAL MACHINES}

4026 This section summarizes a basic interface specification of virtual machines.

4027 4.9.1 IMAGE

4028 To execute virtual machines, an image is needed that specifies the details of the operating system.

\subsubsection{Schema Image}

\begin{tabular}{lll} 
Property & Type & Description \\
\hline name & string & A unique name of the image \\
cloud & string & The name of the cloud \\
label & string & A label that can be defined by the user for the image \\
description & string & A description for the image \\
osType & string & The OS type of the image \\
osVersion & string & The OS version of the image \\
status & string & The status of the image \\
progress & integer & The loading progress percentage of the image \\
visibility & string & The visibility of the image \\
requirement & Requirements & Minimum requirement to run the image
\end{tabular}

\subsubsection{Schema Requirements}

\begin{tabular}{lll} 
Property & Type & Description \\
\hline size & integer & $\begin{array}{l}\text { Minimum disk size in bytes required for the } \\
\text { image }\end{array}$ \\
ram & integer & Minimum ram size in bytes to run the image \\
cpu & string & CPU required to run the image \\
cores & integer & Minimum number of cores
\end{tabular}

\subsubsection{Paths}

\begin{tabular}{lll} 
HTTP & Path & Summary \\
\hline get & $/$ image $/\{$ cloud $\}$ & Returns a list of images for the cloud \\
get & $/$ image $/\{$ cloud $\} /\{$ name $\}$ & Returns the named image \\
put & $/$ image $/\{$ cloud $\} /\{$ name $\}$ & Add an image \\
delete & $/$ image $/\{$ cloud $\} /\{$ name $\}$ & Deletes the named image
\end{tabular}

4032 4.9.1.3.1 limage/\{cloud\}

4033 4.9.1.3.1.1 GET/image/\{cloud\}

4034 Returns a list of all images 
NIST Big DATA INTEROPERABILITY FRAMEWORK: VOLUME 8, REFERENCE ARCHITECTURE INTERFACES

4035

Responses

Code Description Schema

200 The list of images Array[Image]

401 Not authorized String

4036 Parameters

\begin{tabular}{lllll} 
Name & Located in & Description & Required & Schema \\
\hline cloud & path & The name of the cloud & True & String
\end{tabular}

4037 4.9.1.3.2 limage $/\{$ cloud $\} /\{$ name $\}$

4038 4.9.1.3.2.1 GET /image/\{cloud\}/\{name\}

4039 Returns an image by name

4040 Responses

Code Description Schema

200 Returning the information of the image Image

401 Not authorized String

404 The named image could not be found String

4041 Parameters

\begin{tabular}{lllll} 
Name & Located in & Description & Required & Schema \\
\hline cloud & path & The name of the cloud & True & String \\
name & path & The name of the image & True & String
\end{tabular}

4042 4.9.1.3.2.2 PUT /image/\{cloud\}/\{name\}

4043 Sets the named image

4044 Responses

Code Description Schema

200 Image updated or created String

401 Not authorized String

404 The named image could not be found String

4045 Parameters

Name Located in Description Required Schema

cloud path The name of the cloud True String

4046 Request Body

\begin{tabular}{llll} 
Located in & Description & Required & Schema \\
\hline Body & The image to add or modify & True & Image
\end{tabular}


NIST Big DATA INTEROPERABILITY FRAMEWORK: Volume 8, REFERENCE ARCHITECTURE INTERFACES

4047

4048

4049

\subsection{DELETE /image/\{cloud\}/\{name\}}

Deletes an image by name

\section{Responses}

Code Description

200 Deletion successful

401 Not authorized

404 The named image could not be found String

Parameters

\begin{tabular}{lllll} 
Name & Located in & Description & Required & Schema \\
\hline cloud & path & The name of the cloud & True & String \\
name & path & The name of the image & True & String
\end{tabular}

\subsubsection{4 image.yaml}

openapi: "3.0.2"

info:

version: 3.2 .0

x-date: 17-06-2019

$x$-status: defined

title: Image

description: |-

To execute virtual machines, we need an image that specifies the details of the operating system.

termsOfService: "https://github.com/cloudmesh/cloudmesh-nist/blob/master/LICENSE.txt" contact:

name: NIST BDRA Interface Subgroup

url: https://cloudmesh-community.github.io/nist/spec/

license:

name: Apache 2.0

url: https://github.com/cloudmesh/cloudmesh-nist/blob/master/LICENSE.txt servers:

- url: /cloudmesh/v3 paths:

/image/ $\{$ cloud $\}$ :

get:

tags:

- Image

summary: Returns a list of images for the cloud

description: Returns a list of all images

operationId: cloudmesh.image.list

parameters:

- name: cloud

in: path

required: true

schema:

type: string

description: The name of the cloud responses:

'200' :

description: The list of images

content:

application/json:

schema:

type: array

items:

\$ref: '\#/components/schemas/Image ' 


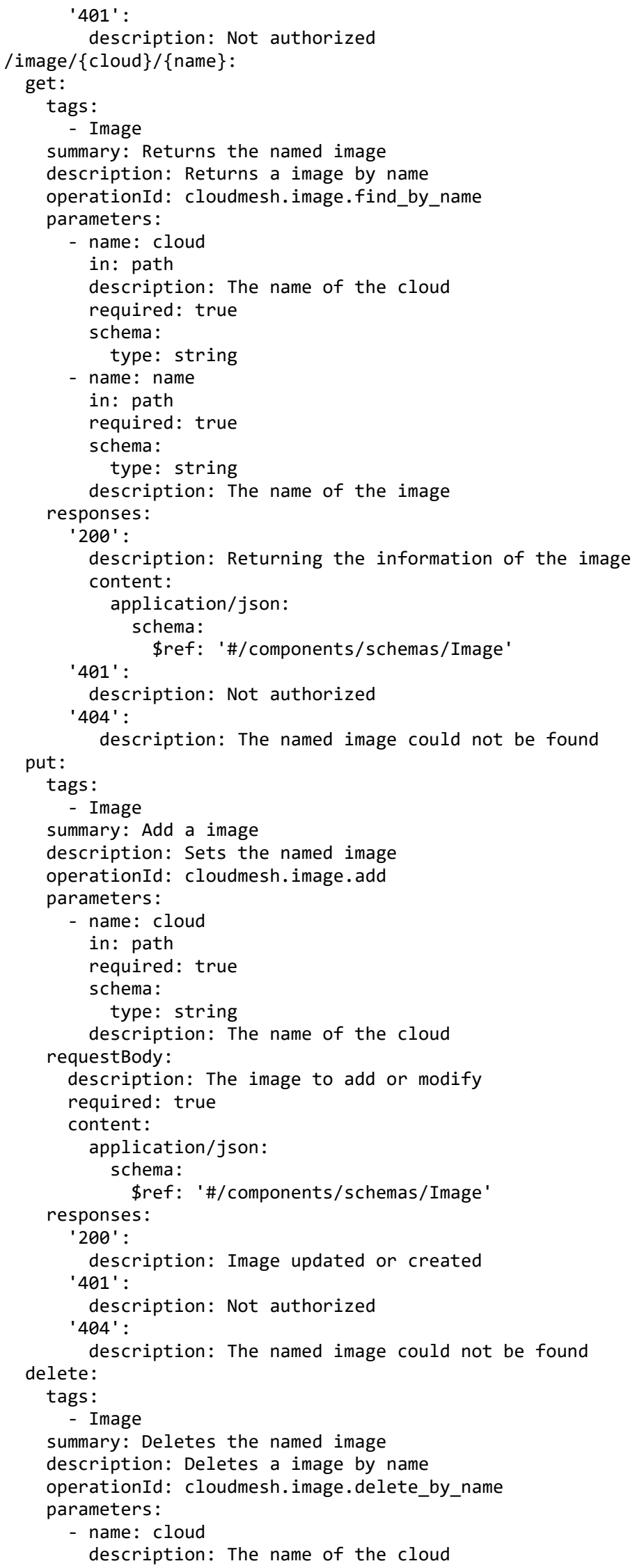


NIST Big DATA INTEROPERABILITY FRAMEWORK: VOLUME 8, REFERENCE ARCHITECTURE INTERFACES

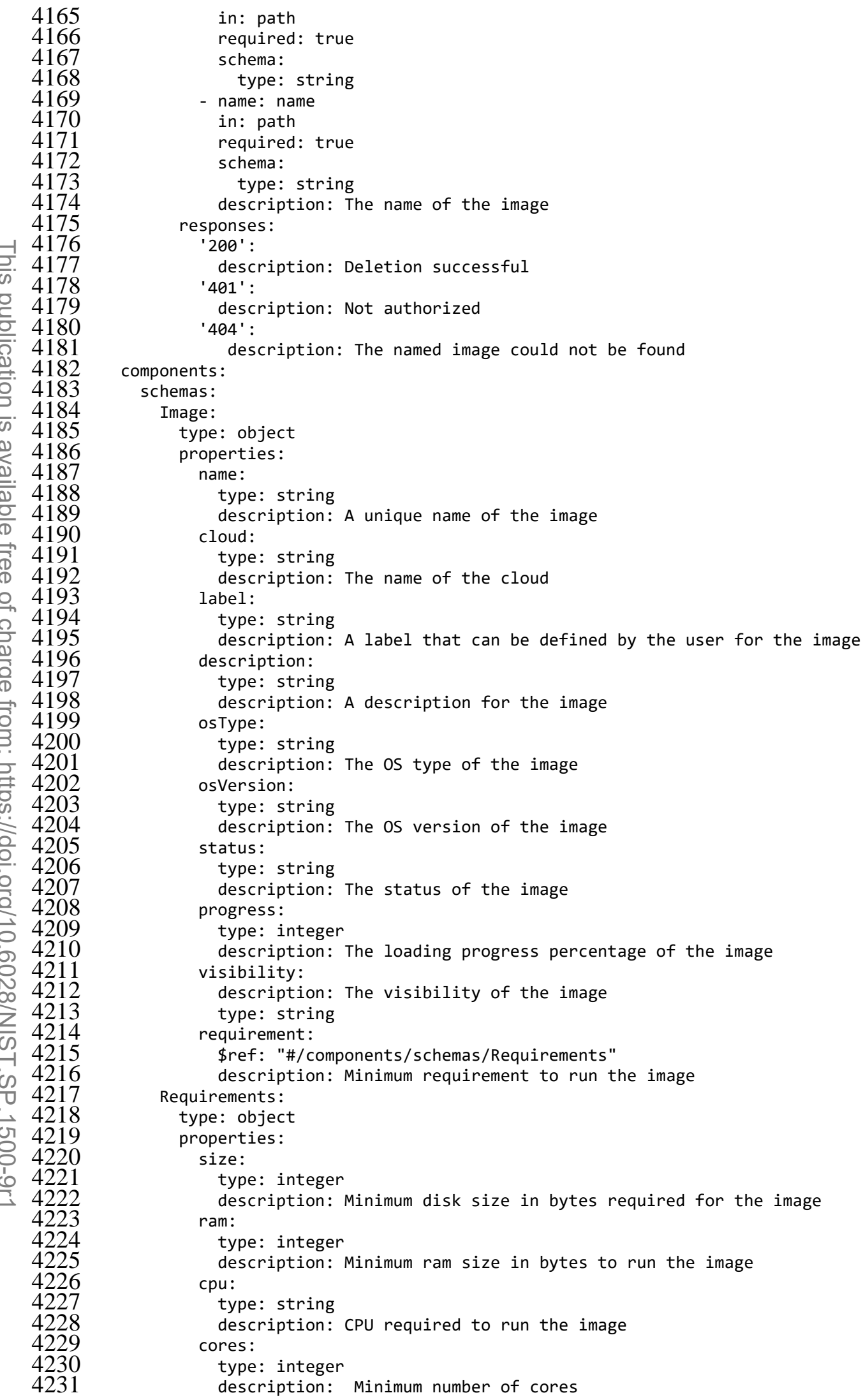




\subsubsection{FLAVOR}

The flavor specifies elementary information about a virtual machine or compute node. This information includes name, ID, label, ram size, swap size, disk space, availability of ephemeral disk, available bandwidth, price value, and cloud name. Flavors and the corresponding information are essential to size a virtual cluster appropriately.

\subsubsection{Schema Flavor}

\begin{tabular}{lll} 
Property & Type & Description \\
\hline name & string & Name of the flavor \\
ld & string & The id of the flavor for the named cloud \\
description & string & A label that a user can set for this flavor \\
ram & string & A description for the flavor \\
swap & integer & Number of bytes used for the image in RAM \\
disk & integer & Number of bytes used for the image in SWAP \\
ephemeral_disk & integer & Number of bytes used for the disk \\
bandwidth & boolean & Specifies whether the flavor features an ephemeral disk \\
price & integer & Bandwidth of the node \\
cloud & number & Price for the flavor \\
& string & Name of the cloud this flavor is used in
\end{tabular}

\subsubsection{Paths}

\begin{tabular}{lll} 
HTTP & Path & Summary \\
\hline get & $/$ flavor $/\{$ cloud $\}$ & Returns a list of flavors for the cloud \\
get & $/$ flavor $/\{$ cloud $\} /$ name $\}$ & Returns the named flavor \\
put & $/$ flavor $/\{$ cloud $\} /$ nname $\}$ & Add a flavor \\
delete & $/$ flavor $/\{$ cloud $\} /$ name $\}$ & Deletes the named flavor
\end{tabular}

\subsection{Iflavor/\{cloud\}}

\subsection{GET/flavor/\{cloud\}}

Returns a list of all flavors

Responses

\begin{tabular}{lll} 
Code & Description & Schema \\
\hline 200 & The list of flavors & Array[Flavor $]$ \\
401 & Not authorized & String
\end{tabular}

4243 Parameters

\begin{tabular}{lllll} 
Name & Located in & Description & Required & Schema \\
\hline cloud & path & The name of the cloud & True & String
\end{tabular}




\section{$4244 \quad 4.9 .2 .2 .2$ Iflavor/\{cloud\}/\{name\}}

\subsection{GET/flavor/\{cloud\}/\{name\}}

4246 Returns a flavor by name

4247 Responses

Code Description Schema

200 Returning the information of the flavor Flavor

401 Not authorized String

404 The named flavor could not be found String

4248 Parameters

\begin{tabular}{lllll} 
Name & Located in & Description & Required & Schema \\
\hline cloud & path & The name of the cloud & True & String \\
name & path & The name of the flavor & True & String
\end{tabular}

4249 4.9.2.2.2.2 PUT /flavor/\{cloud\}/\{name\}

4250 Sets the named flavor

4251 Responses

Code Description Schema

200 Flavor updated String

401 Not authorized String

404 The named flavor could not be found String

4252 Parameters

\begin{tabular}{lllll} 
Name & Located in & Description & Required & Schema \\
\hline cloud & path & The name of the cloud & True & String
\end{tabular}

4253 Request Body

Located in Description Required Schema

Body The flavor to add or modify $\quad$ True $\quad \underline{\text { Flavor }}$

4254 4.9.2.2.2.3 DELETE /flavor/\{cloud\}/\{name\}

4255 Deletes a flavor by name

4256 Responses

\begin{tabular}{lll} 
Code & Description & Schema \\
\hline 200 & Deletion successful & String \\
401 & Not authorized & String \\
404 & The named flavor could not be found & String
\end{tabular}


NIST Big DATA INTEROPERABILITY FRAMEWORK: Volume 8, REFERENCE ARCHITECTURE INTERFACES

4257

Parameters

\begin{tabular}{lllll} 
Name & Located in & Description & Required & Schema \\
\hline cloud & path & The name of the cloud & True & String \\
name & path & The name of the flavor & True & String
\end{tabular}

4258

\subsubsection{3 flavor.yaml}

4259

4260

4261

4262

4263

4264

4265

4266

4267

4268

4269

4270

4271

4272

4273

4274

4275

4276

4277

4278

4279

4280

4281

4282

4283

4284

4285

4286

4287

4288

4289

4290

4291

4292

4293

4294

4295

4296

4297

4298

4299

4300

4301

4302

4303

4304

4305

4306

4307

4308

4309

4310

4311

4312

4313

4314

4315

4316

openapi: "3.0.2"

info:

version: 3.2 .0

$x$-date: $17-06-2019$

$x$-status: defined

title: Flavor

description: |-

The flavor specifies elementary information about a virtual machine

or compute node. This information includes name, id, label, ram size,

swap size, disk space, availability of ephemeral disk, available

bandwidth, price value, cloud name. Flavors and the corresponding

information are essential to size a

virtual cluster appropriately.

termsOfService: "https://github.com/cloudmesh/cloudmesh-nist/blob/master/LICENSE.txt"

contact:

name: NIST BDRA Interface Subgroup

url: https://cloudmesh-community.github.io/nist

license:

name: Apache 2.0

url: https://github.com/cloudmesh/cloudmesh-nist/blob/master/LICENSE.txt

servers:

- url: /cloudmesh/v3

paths:

/flavor/\{cloud\}:

get:

tags:

- Flavor

summary: Returns a list of flavors for the cloud

description: Returns a list of all flavors

operationId: cloudmesh.flavor.list

parameters:

- name: cloud

in: path

required: true

schema:

type: string

description: The name of the cloud

responses:

'200' :

description: The list of flavors

content:

application/json:

schema:

type: array

items:

' 401 ' :

\$ref: '\#/components/schemas/Flavor'

description: Not authorized

/flavor/\{cloud\}/\{name $\}$ :

get:

tags:

- Flavor

summary: Returns the named flavor

description: Returns a flavor by name

operationId: cloudmesh.flavor.find_by_name

parameters: 
NIST Big DATA INTEROPERABILITY FRAMEWORK: Volume 8, REFERENCE ARCHITECTURE INTERFACES

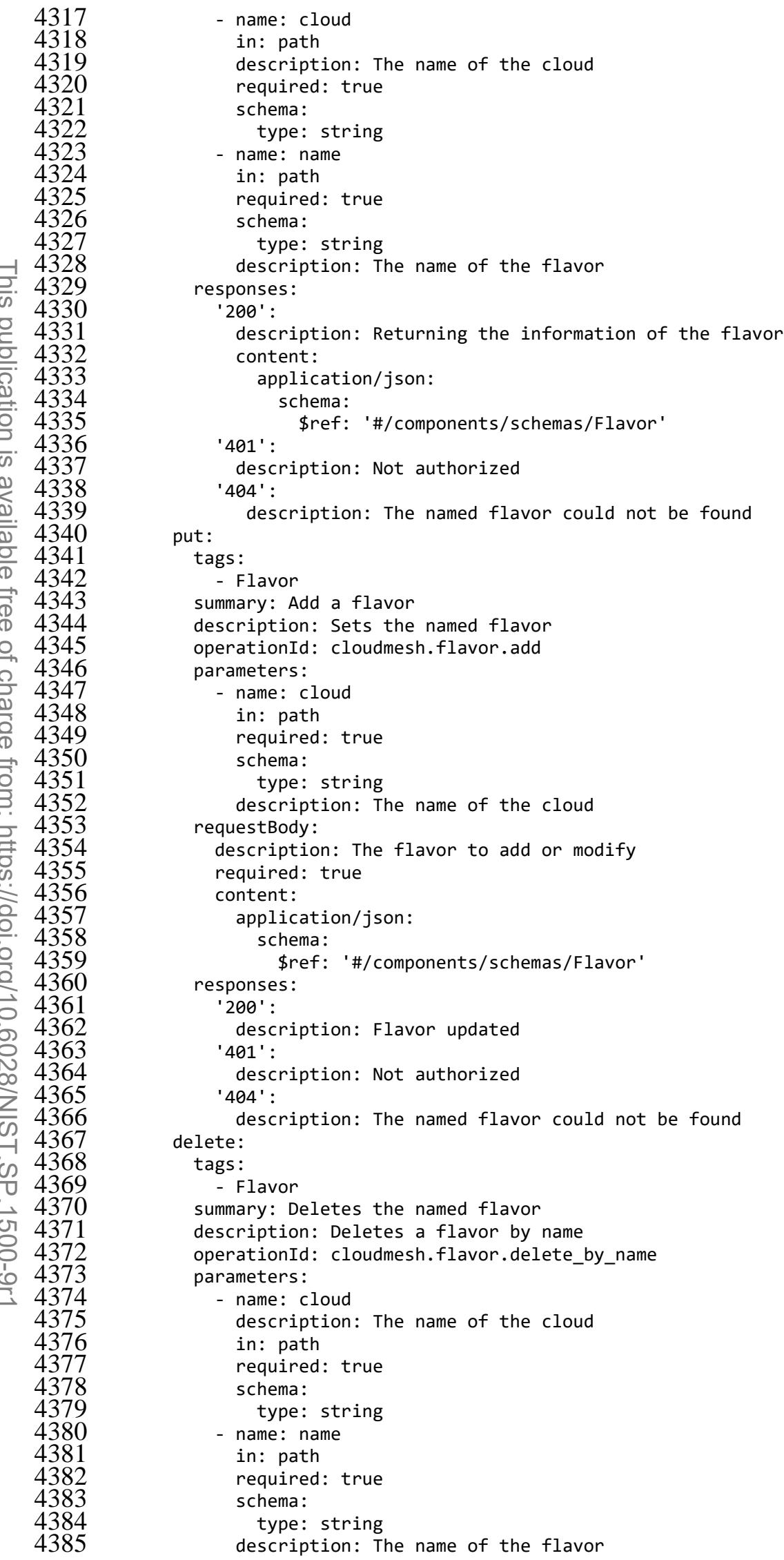


NIST Big DATA INTEROPERABILITY FRAMEWORK: Volume 8, REFERENCE ARCHITECTURE INTERFACES

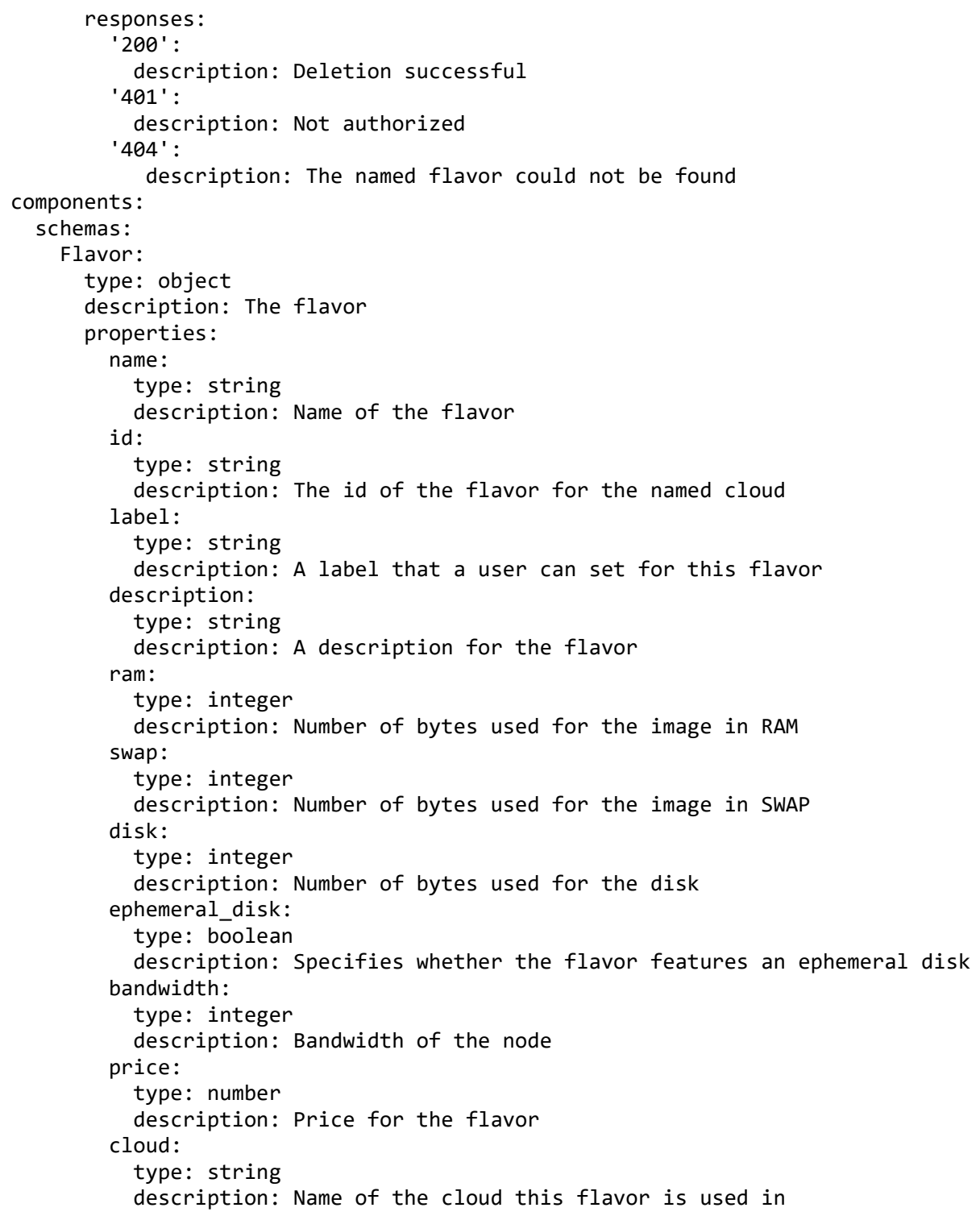

\subsubsection{Virtual Machine}

4432

$4433 \quad \mathrm{VM}$ is used to manage virtual machines. 


\subsubsection{Schema Vm}

\begin{tabular}{lrl} 
Property & Type & Description \\
\hline provider & string & Name of the provider \\
name & string & the unique name of the virtual machine \\
image & string & the image name for the virtual machine \\
flavor & string & the flavor name for the virtual machine \\
region & string & an optional region \\
state & string & The state of the virtual machine \\
private_ips & string & The private IPs \\
public_ips & string & The public IPs \\
metadata & string & $\begin{array}{l}\text { The meta data passed along to the virtual } \\
\text { machine }\end{array}$
\end{tabular}

4.9.3.2 Paths

\begin{tabular}{lll} 
HTTP & Path & Summary \\
\hline get & $/ \mathrm{vm} /\{$ cloud $\}$ & Returns a list of virtual machines for the cloud \\
get & $/ \mathrm{vm} /\{$ cloud $\} /\{$ name $\}$ & Returns the named virtual machine \\
put & $/ \mathrm{vm} /\{$ cloud $\} /\{$ name $\}$ & Add a virtual machine \\
delete & $/ \mathrm{vm} /\{$ cloud $\} /\{$ name $\}$ & Deletes the named virtual machine
\end{tabular}

$4436 \quad 4.9 .3 .2 .1 / \mathrm{vm} /\{$ cloud $\}$

4437 4.9.3.2.1.1 GET/vm/\{cloud\}

4438 Returns a list of all virtual machines

4439 Responses

\begin{tabular}{lll} 
Code & Description & Schema \\
\hline 200 & The list of virtual machines & array[ $\underline{\mathrm{Vm}}]$ \\
400 & No Vm found & String \\
401 & Not authorized & String
\end{tabular}

4440 Parameters

\begin{tabular}{lllll} 
Name & Located in & Description & Required & Schema \\
\hline cloud & path & The name of the cloud & True & String
\end{tabular}

$4441 \quad$ 4.9.3.2.2 $/ \mathrm{vm} /\{$ cloud $\} /\{$ name $\}$

4442 4.9.3.2.2.1 GET/vm/\{cloud\}/\{name\}

4443 Returns a virtual machine by name

4444 Responses 
NIST Big Data INTEROPERABILITY FRAMEWORK: Volume 8, REFERENCE ARCHITECTURE INTERFACES

Code Description Schema

200 Returning the information of the virtual machine $\underline{\text { Vm }}$

400 Error updating virtual machine String

401 Not authorized String

404 The named virtual machine or cloud could not be found String

4445 Parameters

\begin{tabular}{lllll} 
Name & Located in & Description & Required & Schema \\
\hline cloud & path & The name of the cloud & True & String \\
name & path & The name of the virtual machine & True & String
\end{tabular}

4446 4.9.3.2.2.2 PUT /vm/\{cloud\}/\{name\}

4447 Sets the named virtual machine

4448 Responses

\begin{tabular}{lll} 
Code & Description & Schema \\
\hline 200 & Vm updated
\end{tabular}

400 Error updating virtual machine String

401 Not authorized String

404 The named virtual machine or cloud could not be found String

4449 Parameters

\begin{tabular}{lllll} 
Name & Located in & Description & Required & Schema \\
\hline cloud & path & The name of the cloud & True & String
\end{tabular}

4450 Request Body

\begin{tabular}{llll} 
Located in & Description & Required & Schema \\
\hline Body & The virtual machine to add or modify & True & $\underline{\mathrm{Vm}}$
\end{tabular}

4451 4.9.3.2.2.3 DELETE /vm/\{cloud\}/\{name\}

4452 Deletes a virtual machine by name

4453 Responses

\begin{tabular}{lll} 
Code & Description & Schema \\
\hline 200 & Deletion successful & String \\
400 & Error updating virtual machine & String \\
401 & Not authorized & String \\
404 & The named virtual machine or cloud could not be found & String
\end{tabular}


NIST Big DATA INTEROPERABILITY FRAMEWORK: Volume 8, REFERENCE ARCHITECTURE INTERFACES

4454

Parameters

\begin{tabular}{lllll} 
Name & Located in & Description & Required & Schema \\
\hline cloud & path & The name of the cloud & True & String \\
name & path & The name of the virtual machine & True & String
\end{tabular}

4455

- 4456

4457

4458

4459

4460

4461

4462

4463

4464

4465

4466

4467

4468

4469

4470

4471

4472

4473

4474

4475

4476

4477

4478

4479

4480

4481

4482

4483

4484

4485

4486

4487

4488

4489

4490

4491

4492

4493

4494

4495

4496

4497

4498

4499

4500

4501

4502

4503

4504

4505

4506

4507

4508

4509

4510

4511

4512

4513

\subsubsection{3 vm.yaml}

openapi: "3.0.2"

info:

version: 3.2 .0

$x$-date: $17-06-2019$

$x$-status: defined

title: Virtual Machine

description: I-

$\mathrm{Vm}$ is used to manage virtual machines.

termsOfService: https://github.com/cloudmesh-community/nist/blob/master/LICENSE.txt contact:

name: NIST BDRA Interface Subgroup Service

url: https://cloudmesh-community.github.io/nist/spec/

license:

name: Apache 2.0

url: https://github.com/cloudmesh/cloudmesh-nist/blob/master/LICENSE.txt servers:

- url: /cloudmesh/v3

paths:

$/ \mathrm{vm} /\{\mathrm{cloud}\}$ :

get:

tags:

- Vm

summary: Returns a list of virtual machines for the cloud

description: Returns a list of all virtual machines

operationId: cloudmesh.vm.list

parameters:

- name: cloud

in: path

required: true

schema:

type: string

description: The name of the cloud

responses:

'200' :

description: The list of virtual machines

content:

application/json:

schema:

type: array

items:

' 400 ' :

\$ref: '\#/components/schemas/Vm'

description: No Vm found

'401' :

description: Not authorized

$/ \mathrm{vm} /\{\mathrm{cloud}\} /$ name $\}$ :

get:

tags:

- Vm

summary: Returns the named virtual machine

description: Returns a virtual machine by name

operationId: cloudmesh.vm.find_by_name

parameters:

- name: cloud

in: path

description: The name of the cloud 
NIST Big DATA INTEROPERABILITY FRAMEWORK: Volume 8, REFERENCE ARCHITECTURE INTERFACES

4514

4515

4516

4517

4518

4519

4520

4521

4522

4523

4524

거 4525

ㄷ.ᅳ. 4526

क 4527

두 4528

흐 4529

ค 4530

. 4531

을 4532

4533

4534

@ 4535

ฏ. 4536

जे 4537

ㄷ 4538

4539

त् 4540

(ब) 4541

ㅇ 4542

ค 4543

ค. 4544

4545

(1) 4546

त 4547

을 4548

3. 4549

- 4550

4551

( 4552

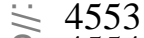

응 4554

ㅇ. 4555

윽 4556

우 4557

ㅇ 4558

') 4559

○ 4560

N 4561

工 4562

4563

- 4564

is 4565

० 4566

$\rightarrow 4567$

다 4568

○ 4569

c 4570

工 4571

4572

4573

4574

4575

4576

4577

4578

4579

4580

4581

4582

required: true

schema:

type: string

- name: name

in: path

description: The name of the virtual machine

required: true

schema:

type: string

responses:

'200' :

description: Returning the information of the virtual machine

content:

application/json:

schema:

'400' :

\$ref: '\#/components/schemas/Vm'

description: Error updating virtual machine

' 401 ' :

description: Not authorized

' 404 ' :

description: The named virtual machine or cloud could not be found

put:

tags:

- Vm

summary: Add a virtual machine

description: Sets the named virtual machine

operationId: cloudmesh.vm.add

parameters:

- name: cloud

in: path

required: true

schema:

type: string

description: The name of the cloud

requestBody:

description: The virtual machine to add or modify

required: true

content:

application/json:

schema:

\$ref: '\#/components/schemas/Vm'

responses:

'200':

description: $\mathrm{Vm}$ updated

'400' :

description: Error updating virtual machine

'401' :

description: Not authorized

'404' :

delete:

description: The named virtual machine or cloud could not be found

tags:

- Vm

summary: Deletes the named virtual machine

description: Deletes a virtual machine by name

operationId: cloudmesh.vm.delete_by_name

parameters :

- name: cloud

description: The name of the cloud

in: path

required: true

schema:

type: string

- name: name

in: path

description: The name of the virtual machine

required: true

schema: 
NIST Big DATA INTEROPERABILITY FRAMEWORK: Volume 8, REFERENCE ARCHITECTURE INTERFACES

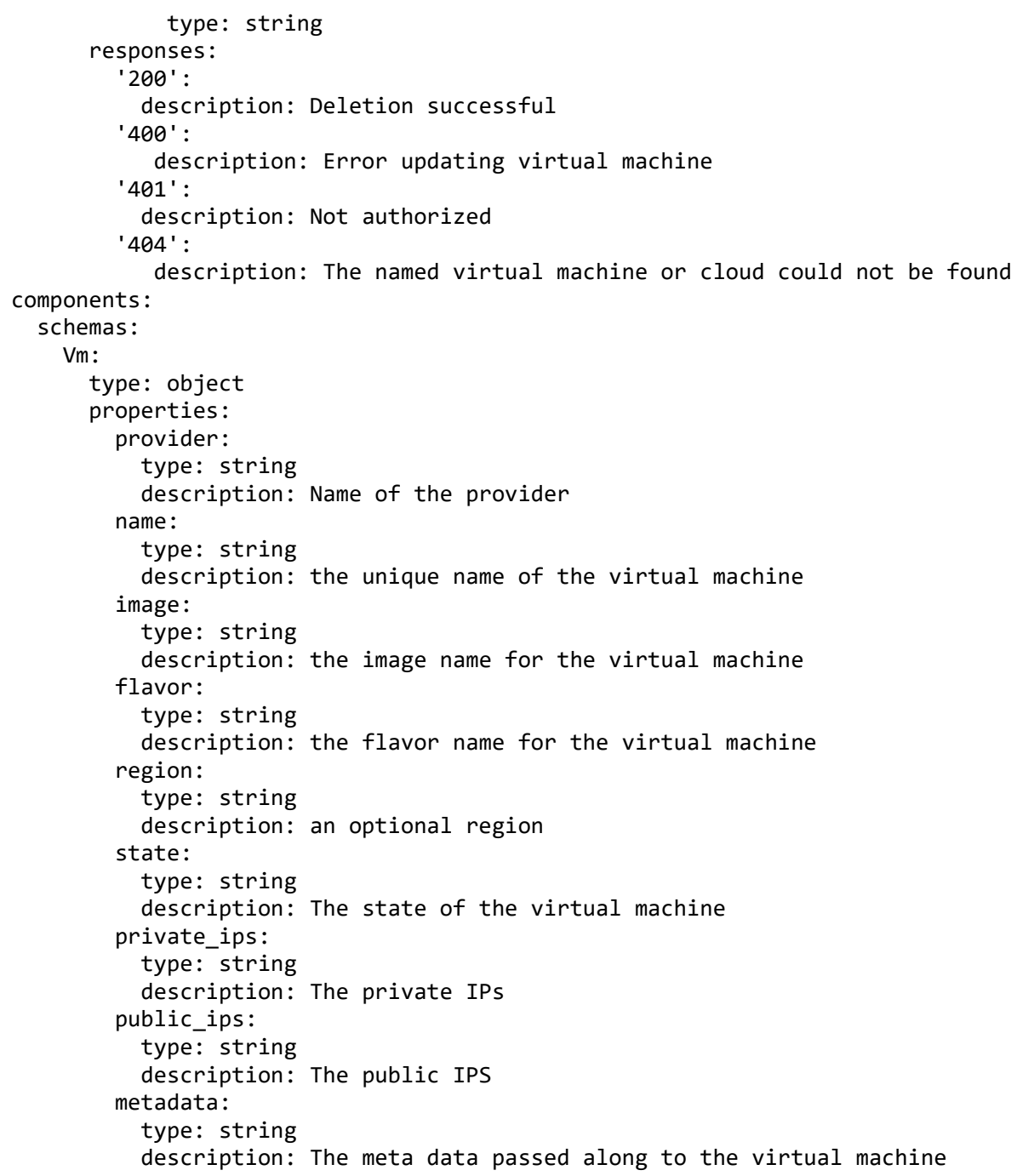

\subsubsection{SECGROUP}

A security group defines the incoming and outgoing security rules which can then be assigned to a node. other possible rules applied on network devices or from the instance's firewall settings. A security group may have one or multiple rules and a node may be associated with one or more security groups.

\subsubsection{Schema Secgroup}

\begin{tabular}{lll} 
Property & Type & Description \\
\hline name & String & Name of the security group \\
description & String & Describes what the security group is for \\
rules & array[SecGroupRule] & List of Security group rules
\end{tabular}




\subsubsection{Schema SecGroupRule}

\begin{tabular}{|c|c|c|}
\hline Property & Туре & Description \\
\hline name & String & Unique name of the rule \\
\hline ingress & boolean & The defined security group rule is for ingress if True \\
\hline egress & boolean & The defined security group rule is for egress if True \\
\hline remote_group & String & Name of the group if the rule is defined by group instead of IP range \\
\hline protocol & String & $\begin{array}{l}\text { The protocol used such as Transmission Control Protocol (TCP), User } \\
\text { Datagram Protocol (UDP), Internet Control Message Protocol (ICMP) }\end{array}$ \\
\hline from_port & integer & Port range starting port \\
\hline to_port & integer & Port range ending port \\
\hline cidr & String & $\begin{array}{l}\text { The source or destination network in Classless Inter-Domain Routing } \\
\text { (CIDR) notation }\end{array}$ \\
\hline
\end{tabular}

\subsubsection{Paths}

\begin{tabular}{lll} 
HTTP & Path & Summary \\
\hline get & $/$ secgroup & Returns all security groups \\
get & $/$ secgroup $/\{$ name $\}$ & Return the security group by name \\
post & $/$ secgroup $/\{$ name $\}$ & Create the named security group \\
get & $/$ secgroup $/\{$ name $\} /$ rule $/\{$ rule $\}$ & Get an existing rule from the specified security group \\
put & $/$ secgroup $/\{$ name $\} /$ rule $/\{$ rule $\}$ & Create or update specified security group \\
delete & $/$ secgroup $/\{$ name $\} /$ rule $/\{$ rule $\}$ & Delete an existing rule from the specified security group
\end{tabular}

$4634 \quad$ 4.9.4.3.1 /secgroup

4635 4.9.4.3.1.1 GET/secgroup

4636 Returns all security groups

4637 Responses

\begin{tabular}{lll} 
Code & Description & Schema \\
\hline 200 & security group information & array[Secgroup] \\
401 & Not authorized & String
\end{tabular}

4638 4.9.4.3.2 Isecgroup/\{name\}

\subsection{GET/secgroup/\{name\}}

4640 Return the security group by name 
Responses

\begin{tabular}{lll} 
Code & Description & Schema \\
\hline 200 & Security group information & Secgroup \\
401 & Not authorized & String \\
404 & The named security group could not be found & String
\end{tabular}

4642 Parameters

\begin{tabular}{lllll} 
Name & Located in & Description & Required & Schema \\
\hline name & path & Name of the security group & True & String
\end{tabular}

4643 4.9.4.3.2.2 POST/secgroup/\{name\}

4644 Create a new named security group

4645 Responses

\begin{tabular}{lll} 
Code & Description & Schema \\
\hline 201 & Created & String \\
400 & The group could not be created & String \\
401 & Not authorized & String
\end{tabular}

4646 Parameters

\begin{tabular}{lllll} 
Name & Located in & Description & Required & Schema \\
\hline name & path & The name of the security group to create & True & String
\end{tabular}

4647 4.9.4.3.3 /secgroup/\{name\}/rule/\{rule\}

4648 4.9.4.3.3.1 GET/secgroup/\{name\}/rule/\{rule\}

4649 Create a new rule in security group

4650 Responses

Schema

200 The security group rule definition info

Secgrouprule

401 Not authorized

String

404 The named security group or role could not be found String

4651 Parameters

\begin{tabular}{lllll} 
Name & $\begin{array}{l}\text { Located } \\
\text { in }\end{array}$ & Description & Required & Schema \\
\hline name & path & $\begin{array}{l}\text { The name of the security group from which the rule will be } \\
\text { deleted }\end{array}$ & True & String \\
rule & path & $\begin{array}{l}\text { The rule to be added } \\
\text { True }\end{array}$ & String
\end{tabular}


NIST Big DATA INTEROPERABILITY FRAMEWORK: VOLUME 8, REFERENCE ARCHITECTURE INTERFACES

\subsection{PUT /secgroup/\{name\}/rule/\{rule\}}

4653 Create a new rule in security group

4654 Responses

Code Description Schema

200 Created String

401 Not authorized String

404 The named security group or role could not be found String

Parameters

Name Located in Description Required Schema

name path The name of the new security group to create True String

4656 Request Body

Located in Description Required Schema

Body The new security group rule to create True $\quad \underline{\text { SecGroupRule }}$

4.9.4.3.3.3 DELETE /secgroup/\{name\}/rule/\{rule\}

4658 Create a new rule in security group

4659 Responses

Code Description Schema

200 Deleted successfully String

401 Not authorized String

404 The named security group or role could not be found String

Parameters

\begin{tabular}{lllll} 
Name & Located in & Description & Required & Schema \\
\hline name & path & The named secgroup & True & String \\
rule & path & The secgroup rule & True & String
\end{tabular}

\subsubsection{4 secgroup.yaml}

openapi: "3.0.2"

info:

version: 3.2 .0

x-date: 17-06-2019

$x$-status: defined

title: Secgroup

description: |-

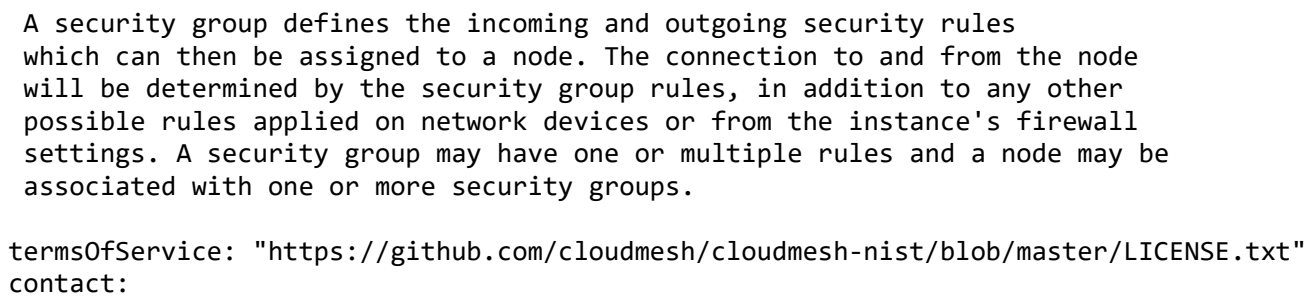


NIST Big DATA INTEROPERABILITY FRAMEWORK: Volume 8, REFERENCE ARCHITECTURE INTERFACES

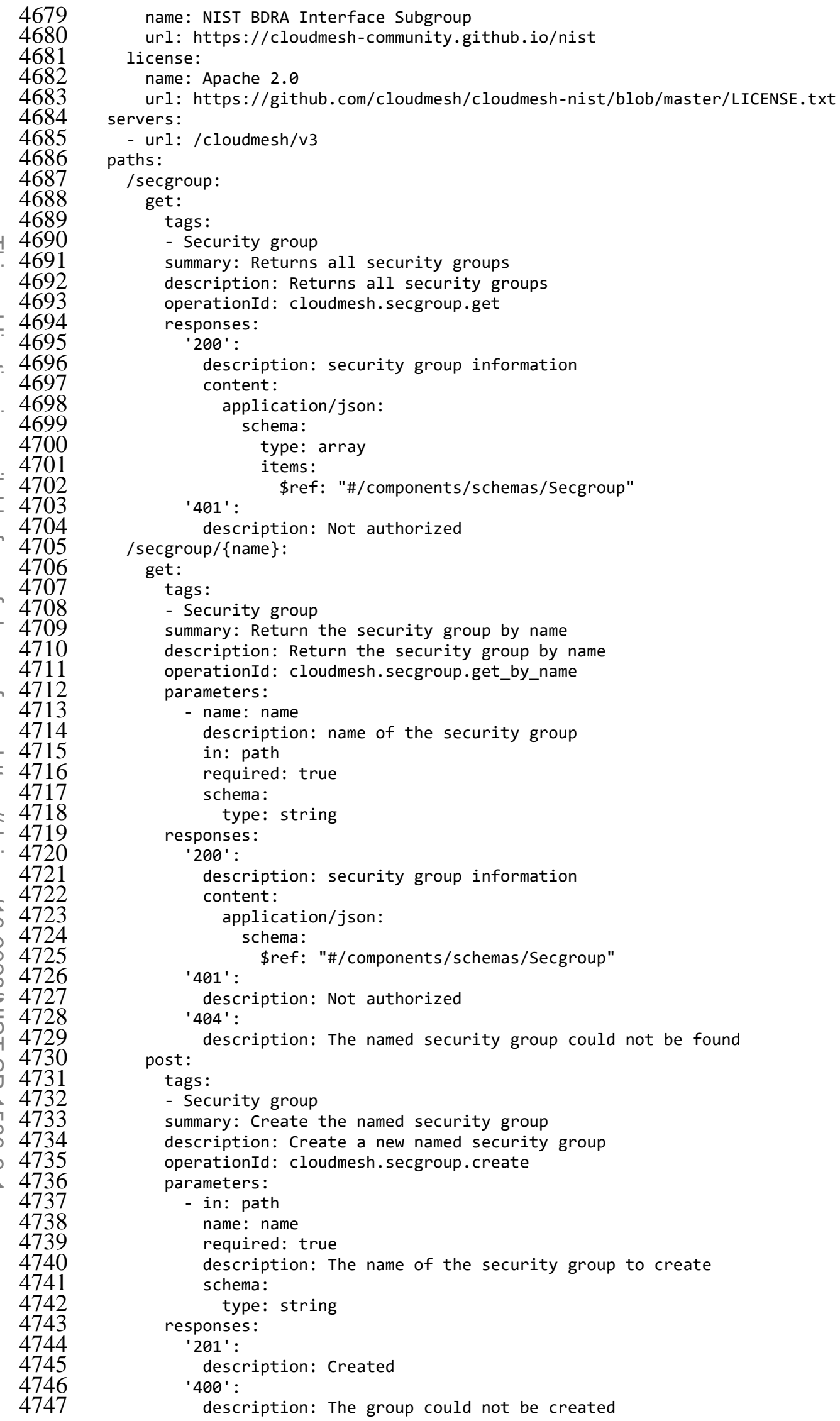




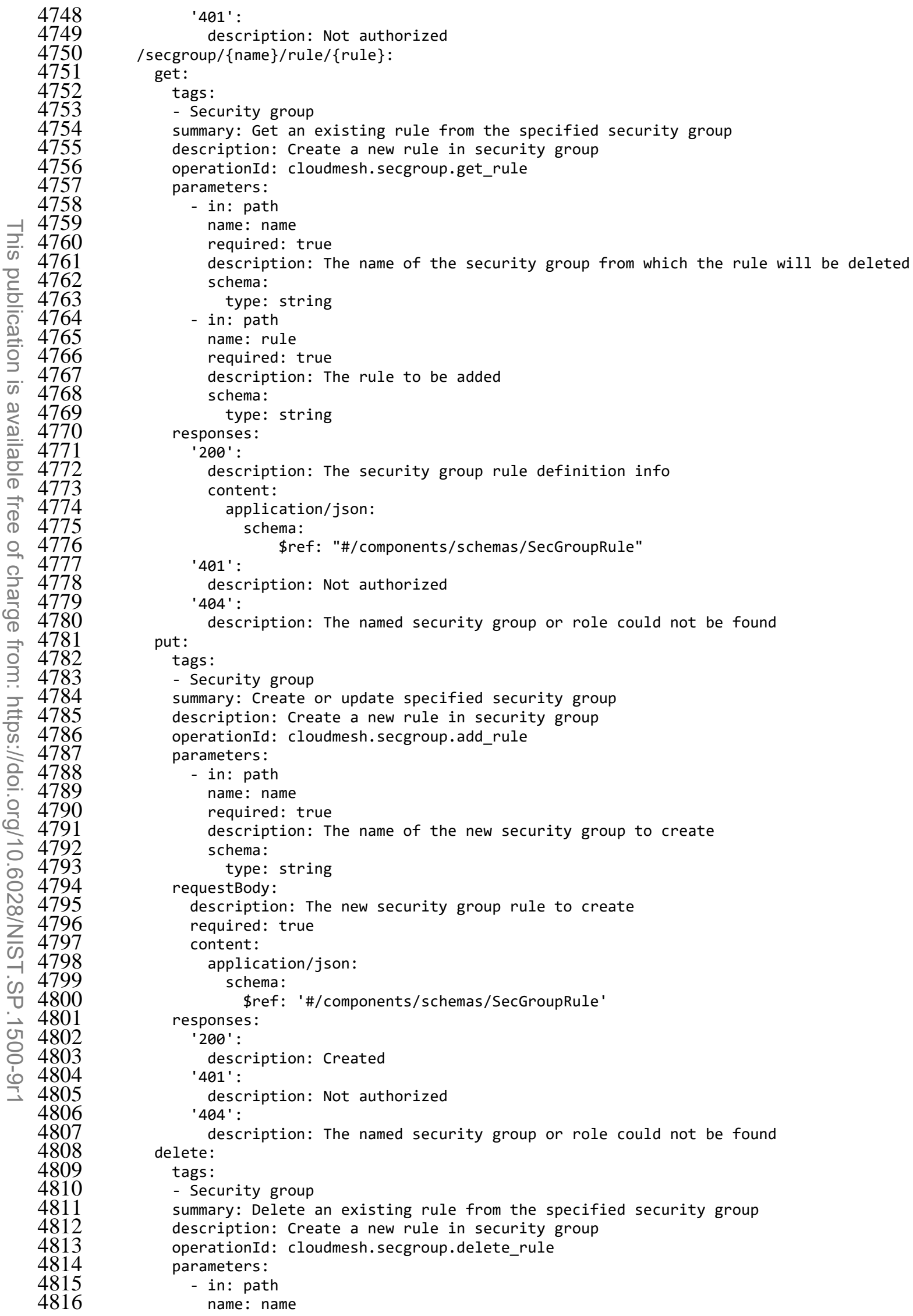


NIST Big DATA INTEROPERABILITY FRAMEWORK: VOLUME 8, REFERENCE ARCHITECTURE INTERFACES

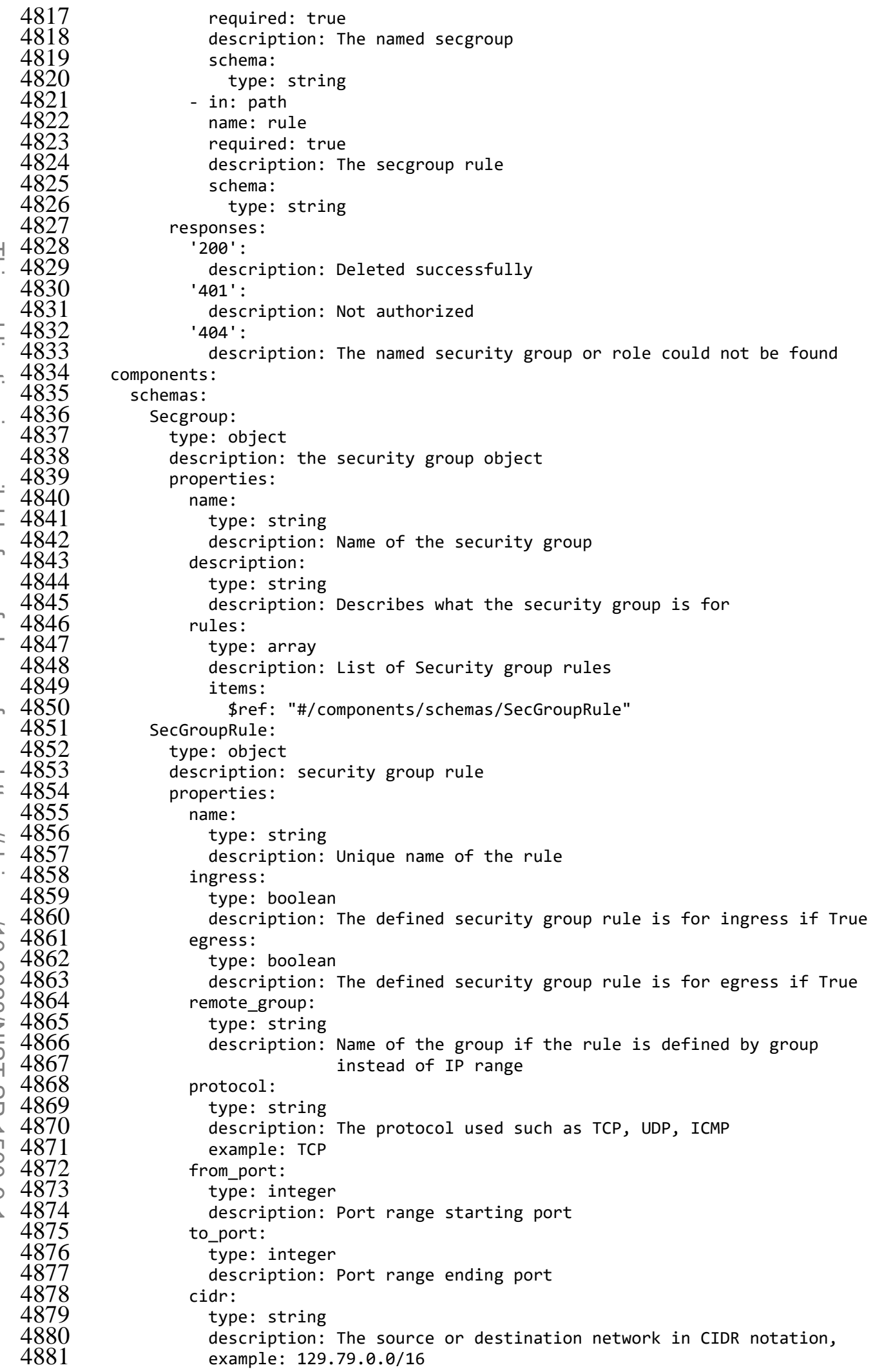


$4882 \quad 4.9 .5$ NIC

4883 A resource store Network Interface Controller (NIC) information.

$4884 \quad$ 4.9.5.1 Schema Nic

\begin{tabular}{lll} 
Property & Type & Description \\
\hline name & string & Name of the network interface controller \\
kind & string & Kind of the network interface controller (e.g., Wi-Fi, \\
& & WAN) \\
mac & string & The mac address \\
ip & string & The IP address \\
mask & string & The network mask \\
broadcast & string & The broadcast address \\
gateway & string & The gateway address \\
mtu & integer & The maximum transmission unit (MTU) of the NIC \\
bandwidth & integer & The bandwidth in bps
\end{tabular}

\subsubsection{Paths}

\begin{tabular}{lll} 
HTTP & Path & Summary \\
\hline get & $/$ nic & Returns a list of network interface controllers \\
get & $/$ nic $/\{$ name $\}$ & Returns the named network interface controller \\
put & $/$ nic $/\{$ name $\}$ & Set a network interface controller \\
delete & $/$ nic $/\{$ name $\}$ & Deletes the named network interface controller
\end{tabular}

$4886 \quad 4.9 .5 .2 .1$ Inic

4887 4.9.5.2.1.1 GET /nic

4888 Returns a list of all network interface controllers

4889 Responses

\begin{tabular}{lll} 
Code & Description & Schema \\
\hline 200 & The list of network interface controllers & array[Nic] \\
401 & Not authorized & String
\end{tabular}

$4890 \quad 4.9 .5 .2 .2$ Inic/\{name $\}$

4891 4.9.5.2.2.1 GET/nic/\{name\}

4892 Returns a network interface controller by name 
NIST Big DATA INTEROPERABILITY FRAMEWORK: Volume 8, REFERENCE ARCHITECTURE INTERFACES

4893

Responses

Code Description

Schema

200 Returning the information of the network interface controller Nic

401 Not authorized

String

404 The named network interface controller could not be found String

Parameters

\begin{tabular}{lllll} 
Name & Located in & Description & Required & Schema \\
\hline name & path & The name of the network interface controller & True & String
\end{tabular}

\subsection{PUT /nic/\{name\}}

4896 Sets the named network interface controller

Responses

Code Description Schema

$200 \quad$ NIC updated String

401 Not authorized String

4898 Request Body

Located in Description Required Schema

Body The new NIC to create or update True $\quad \underline{\text { Nic }}$

\subsection{DELETE /nic/\{name\}}

4900 Deletes a network interface controller by name

4901 Responses

Code Description Schema

200 Deletion successful
String

401 Not authorized String

404 The named network interface controller could not be found String

Parameters

\begin{tabular}{lllll} 
Name & Located in & Description & Required & Schema \\
\hline name & path & The name of the network interface controller & True & String
\end{tabular}

\subsubsection{3 nic.yaml}

openapi: "3.0.2"

info:

version: 3.2 .0

$x$-date: 17-06-2019

$x$-status: defined

title: Nic

description: |-

A resource store Network Interface Controller (NIC) information. 


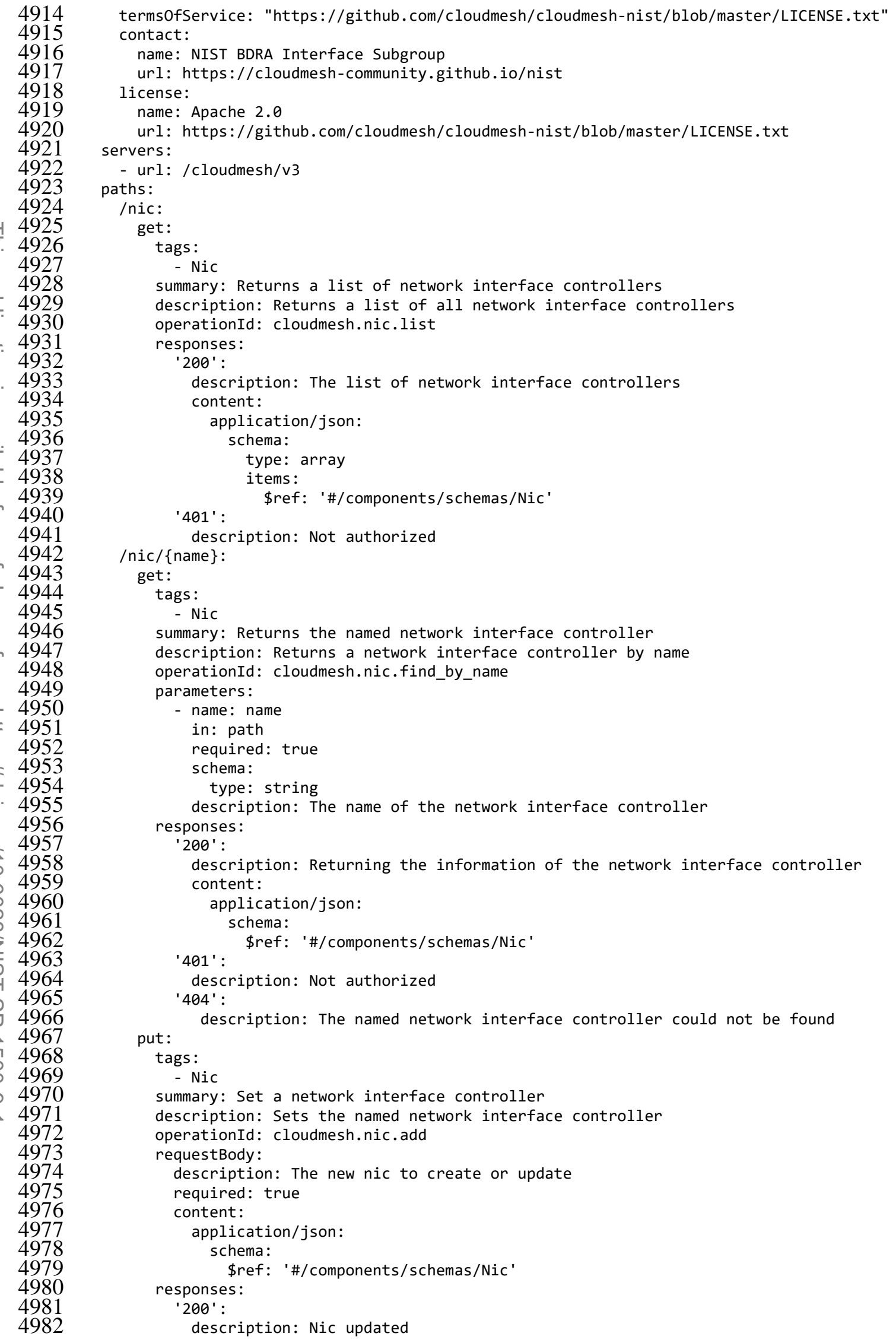


NIST Big DATA INTEROPERABILITY FRAMEWORK: VOLUME 8, REFERENCE ARCHITECTURE INTERFACES

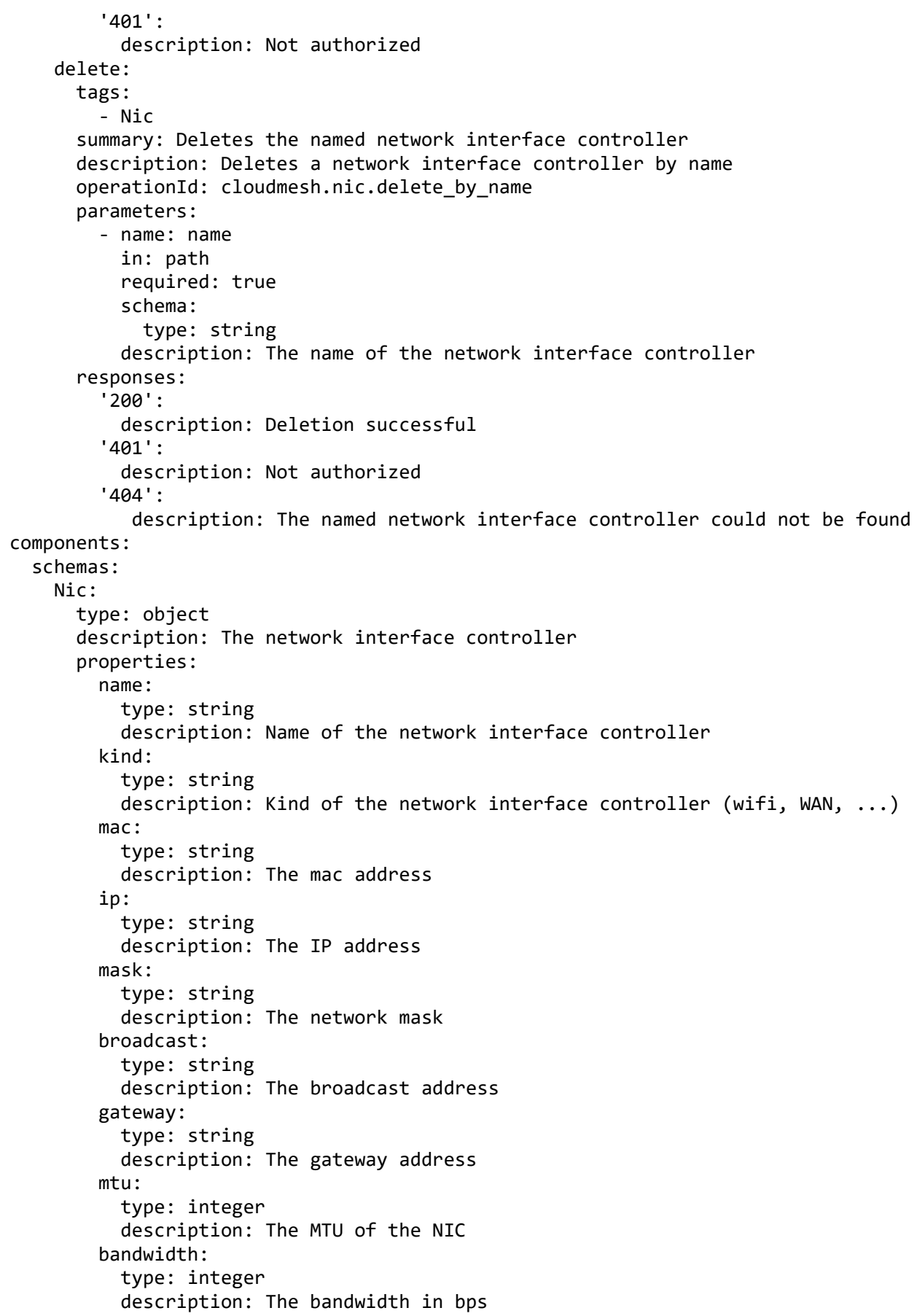

\subsubsection{CONTAINERS}

5040 Numerous different containers are likely to be created and handling them becomes more and more time 5041 consuming as their number increases. This service helps to solve that issue by storing containers and their 5042 corresponding information. 


\subsubsection{Schema Container}

\begin{tabular}{lll} 
Property & Type & Description \\
\hline name & string & Name of the container \\
version & string & Version of the container \\
label & string & Label of the container \\
type & string & Type of the container \\
definition & string & Definition or manifest of the container \\
imgURI & string & URI of the container \\
tags & array[string] & Tags of the container
\end{tabular}

5044 4.10.1.2 Paths

\begin{tabular}{lll} 
HTTP & Path & Summary \\
\hline get & /container & Returns a list of containers \\
get & /container/\{name $\}$ & Returns the named container \\
put & $/$ container $/\{$ name $\}$ & Set an container \\
delete & /container $/\{$ name $\}$ & Deletes the named container
\end{tabular}

$5045 \quad 4.10 .1 .2 .1 / c o n t a i n e r$

5046 4.10.1.2.1.1 GET/container

5047 Returns a list of all containers

5048 Responses

\begin{tabular}{lll} 
Code & Description & Schema \\
\hline 200 & The list of containers & array[Container] \\
401 & Not authorized & String
\end{tabular}

$5049 \quad$ 4.10.1.2.2/container/\{name\}

5050 4.10.1.2.2.1 GET/container/\{name\}

5051 Returns a container by name

5052 Responses

\begin{tabular}{lll} 
Code & Description & Schema \\
\hline 200 & Returning the information of the container & Container \\
400 & No Container found & String \\
401 & Not authorized & String \\
404 & The named container could not be found & String
\end{tabular}


NIST Big DATA INTEROPERABILITY FRAMEWORK: VOLUME 8, REFERENCE ARCHITECTURE INTERFACES

Parameters

\begin{tabular}{lllll} 
Name & Located in & Description & Required & Schema \\
\hline name & path & The name of the container & True & String
\end{tabular}

\subsection{PUT /container/\{name\}}

5055 Sets the named container

5056 Responses

Code Description Schema

200 Container updated String

401 Not authorized String

400 Error updating container String

5057 Request Body

Located in Description Required Schema

Body The new container to create True $\underline{\text { Container }}$

5058 4.10.1.2.2.3 DELETE /container/\{name\}

5059 Deletes a container by name

5060 Responses

Code Description Schema

200 Deletion successful String

401 Not authorized String

404 The named container could not be found String

5061 Parameters

\begin{tabular}{lllll} 
Name & Located in & Description & Required & Schema \\
\hline name & path & The name of the container & True & String
\end{tabular}

4.10.1.3 containers.yaml

openapi: "3.0.2"

info:

version: 3.2 .0

x-date: 17-06-2019

$x$-status: defined

title: Containers

description: |-

Numerous different containers are likely to be created and handling them

becomes more and more time consuming as their number increases. This service

helps to solve that issue by storing containers and their corresponding

information.

termsOfService: "https://github.com/cloudmesh/cloudmesh-nist/blob/master/LICENSE.txt"

contact:

name: NIST BDRA Interface Subgroup

url: https://cloudmesh-community.github.io/nist

license: 
NIST Big DATA INTEROPERABILITY FRAMEWORK: Volume 8, REFERENCE ARCHITECTURE INTERFACES

5081

5082

5083

5084

5085

5086

5087

5088

5089

5090

5091

5092

5093

5094

5095

5096

5097

5098

5099

5100

5101

5102

5103

5104

5105

5106

5107

5108

5109

5110

5111

5112

5113

5114

5115

5116

5117

5118

5119

5120

5121

5122

5123

5124

5125

5126

5127

5128

5129

5130

5131

5132

5133

5134

5135

5136

5137

5138

5139

5140

5141

5142

5143

5144

5145

5146

5147

5148

5149

name: Apache 2.0

url: https://github.com/cloudmesh/cloudmesh-nist/blob/master/LICENSE.txt servers:

- url: /cloudmesh/v3

paths:

/container:

get:

tags:

- Container

summary: Returns a list of containers

description: Returns a list of all containers

operationId: cloudmesh.container.list

responses:

'200' :

description: The list of containerses

content:

application/json:

schema:

type: array

items:

'401' :

\$ref: '\#/components/schemas/Container'

description: Not authorized

/container/ $\{$ name $\}$ :

get:

tags:

- Container

summary: Returns the named container

description: Returns an container by name

operationId: cloudmesh.container.find_by_name

parameters:

- name: name

in: path

required: true

schema:

type: string

description: The name of the container

responses:

' 200 ' :

description: Returning the information of the container

content:

application/json:

schema:

'400' :

\$ref: '\#/components/schemas/Container'

description: No Container found

'401' :

description: Not authorized

'404' :

description: The named container could not be found

put:

tags:

- Container

summary: Set an container

description: Sets the named container

operationId: cloudmesh.container.add

requestBody:

description: The new container to create

required: true

content:

application/json:

schema:

responses:

\$ref: '\#/components/schemas/Container'

'200' :

description: Container updated

'401' :

description: Not authorized

'400' : 
NIST Big DATA INTEROPERABILITY FRAMEWORK: VOLUME 8, REFERENCE ARCHITECTURE INTERFACES

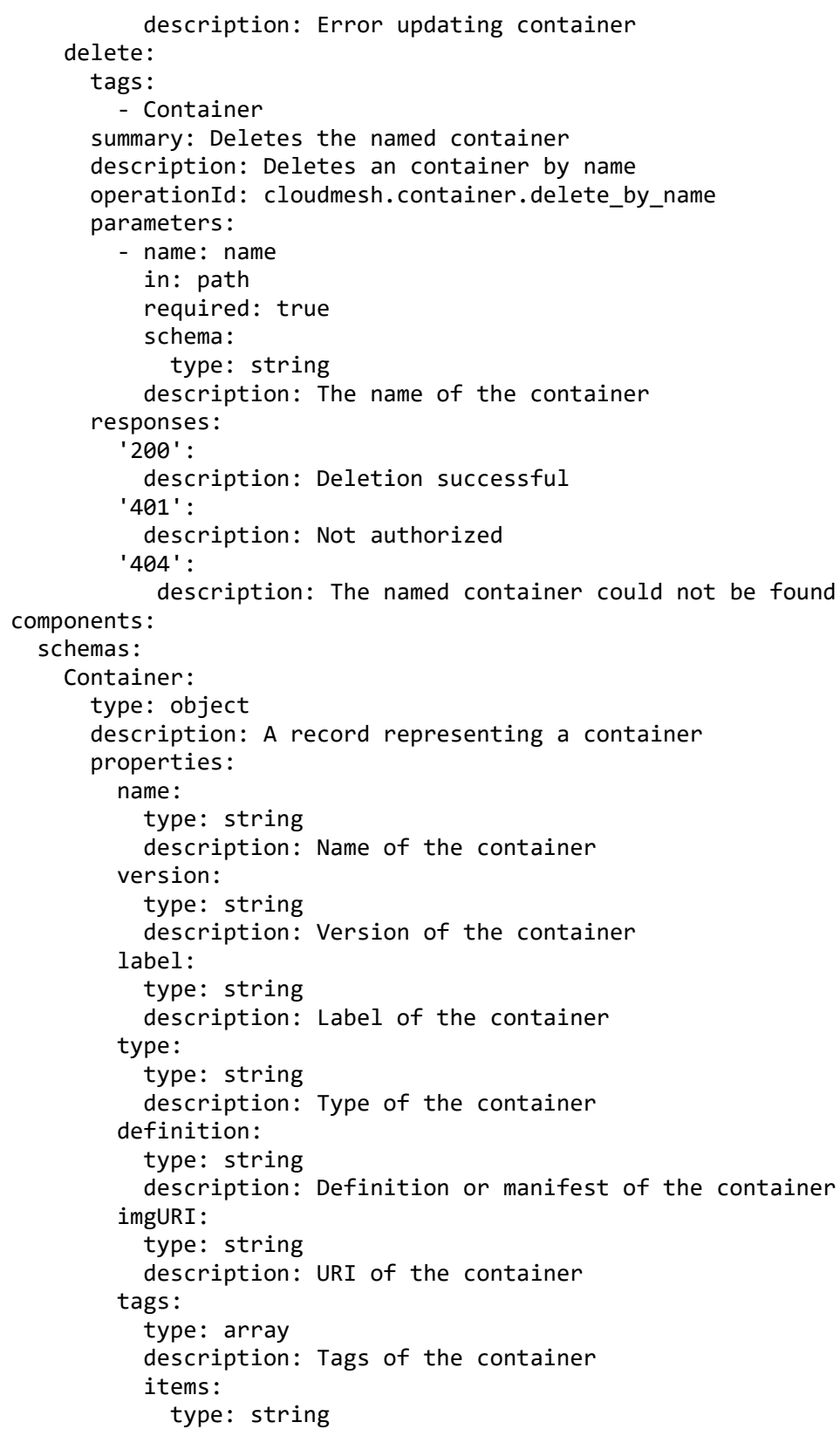

5200 4.11 COMPUTE MANAGEMENT: MAPREDUCE

\section{$5201 \quad$ 4.11.1 MAPREDUCE}

5202 A service to store the information of a MapReduce deployment definition. All of the attributes are stored 5203 as Strings. 


\subsubsection{Schema Map}

\begin{tabular}{lll} 
Property & Type & Description \\
\hline name & string & The name of the map function \\
kind & string & The kind in which the specification is provided \\
content & string & The kind in which the specification is provided
\end{tabular}

\subsubsection{Schema Reduce}

\begin{tabular}{lrl} 
Property & Type & Description \\
\hline name & string & The name of the reduce function \\
kind & string & The kind in which the specification is provided \\
content & string & The kind in which the specification is provided
\end{tabular}

5206 4.11.1.3 Schema Data

\begin{tabular}{lll} 
Property & Type & Description \\
\hline name & string & The name of the data \\
content & string & The content of the data
\end{tabular}

$5207 \quad$ 4.11.1.4 Paths

\begin{tabular}{lll} 
HTTP & Path & Summary \\
\hline get & /mapreduce & Returns the data identified by the MapReduce resource \\
get & $/$ mapreduce/ $\{$ name $\}$ & Returns the data identified by the map and function \\
put & $/$ mapreduce/map/ $\{$ name $\}$ & Create or update the map function \\
get & $/$ mapreduce/map/ $\{$ name $\}$ & Returns the data identified by the map function \\
put & $/$ mapreduce/reduce/ $\{$ name $\}$ & Create or update the reduce function \\
get & /mapreduce/reduce/ $\{$ name $\}$ & Returns the data identified by the reduce function
\end{tabular}

5208 4.11.1.4.1/mapreduce

5209 4.11.1.4.1.1 GET/mapreduce

5210 Returns the data identified by the MapReduce resource

5211 Responses

\begin{tabular}{lll} 
Code & Description & Schema \\
\hline 200 & MapReduce names & array[String] \\
401 & Not authorized & String
\end{tabular}


$5212 \quad$ 4.11.1.4.2/mapreduce $/\{$ name\}

5213 4.11.1.4.2.1 GET/mapreduce/\{name\}

5214 Returns the data identified by the reduce function.

5215 Responses

\begin{tabular}{lll} 
Code & Description & Schema \\
\hline 200 & The data identified by reduce & array[Data] \\
401 & Not authorized & String \\
404 & The resource could not be found & String
\end{tabular}

5216 Parameters

\begin{tabular}{lllll} 
Name & Located in & Description & Required & Schema \\
\hline name & path & The name of the function & True & String
\end{tabular}

5217 4.11.1.4.3/mapreduce/map/\{name\}

5218 4.11.1.4.3.1 PUT/mapreduce/map/\{name\}

5219 Create or update the map function

5220 Responses

\begin{tabular}{lll} 
Code & Description & Schema \\
\hline 200 & The map function was created or updated & String \\
401 & Not authorized & String \\
404 & The resource could not be found & String
\end{tabular}

5221 Parameters

\begin{tabular}{lllll} 
Name & Located in & Description & Required & Schema \\
\hline name & path & The name of the function & True & String
\end{tabular}

5222 Request Body

\begin{tabular}{llll} 
Located in & Description & Required & Schema \\
\hline Body & The new default to create & True & Map
\end{tabular}

5223 4.11.1.4.3.2 GET/mapreduce/map/\{name\}

5224 Returns the data identified by the map function

5225 Responses

\begin{tabular}{lll} 
Code & Description & Schema \\
\hline 200 & The data identified by map & array[Data] \\
401 & Not authorized & String \\
404 & The resource could not be found & String
\end{tabular}


NIST Big DATA INTEROPERABILITY FRAMEWORK: VOLUME 8, REFERENCE ARCHITECTURE INTERFACES

Parameters

\begin{tabular}{lllll} 
Name & Located in & Description & Required & Schema \\
\hline name & path & The name of the function & True & String
\end{tabular}

4.11.1.4.4/mapreduce/reduce $/\{$ name $\}$

5228 4.11.1.4.4.1 PUT /mapreduce/reduce/\{name\}

5229 Create or update the reduce function

5230 Responses

\begin{tabular}{lll} 
Code & Description & Schema \\
\hline 200 & The reduce function was created or updated & String \\
401 & Not authorized & String \\
404 & The resource could not be found & String
\end{tabular}

5231 Parameters

\begin{tabular}{lllll} 
Name & Located in & Description & Required & Schema \\
\hline name & path & The name of the function & True & String
\end{tabular}

5232 Request Body

\begin{tabular}{llll} 
Located in & Description & Required & Schema \\
\hline Body & The new default to create & True & Reduce
\end{tabular}

\subsection{GET/mapreduce/reduce/\{name\}}

5234 Returns the data identified by the reduce function

5235 Responses

\begin{tabular}{lll} 
Code & Description & Schema \\
\hline 200 & The data identified by reduce & array[Data] \\
401 & Not authorized & String \\
404 & The resource could not be found & String
\end{tabular}

Parameters

\begin{tabular}{lllll} 
Name & Located in & Description & Required & Schema \\
\hline name & path & The name of the function & True & String
\end{tabular}

\subsubsection{5 mapreduce.yaml}

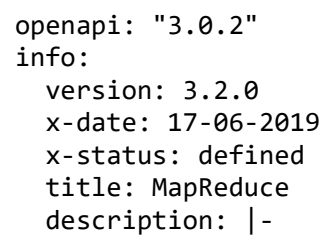


NIST Big DATA INTEROPERABILITY FRAMEWORK: VOLUME 8, REFERENCE ARCHITECTURE INTERFACES

All of the attributes are stored as Strings.

termsOfService: "https://github.com/cloudmesh/cloudmesh-nist/blob/master/LICENSE.txt" contact:

name: NIST BDRA Interface Subgroup

url: https://cloudmesh-community.github.io/nist

license:

name: Apache 2.0

url: https://github.com/cloudmesh/cloudmesh-nist/blob/master/LICENSE.txt servers:

- url: /cloudmesh/v3 paths:

/mapreduce:

get:

tags:

- mapreduce

summary: Returns the data identified by the mapreduce resource

description: Returns the data identified by the mapreduce resource

operationId: cloudmesh.mapreduce.list

responses:

'200' :

description: mapreduce names

content:

application/json:

schema:

type: array

items:

'401' :

type: string

description: Not authorized

/mapreduce/ $\{$ name $\}$ :

get:

tags:

- mapreduce

summary: Returns the data identified by the map and function

description: Returns the data identified bythe reduce function.

operationId: cloudmesh.mapreduce.get

parameters :

- name: name

in: path

required: true

schema:

type: string

description: The name of the function responses:

' 200 ' :

description: The data identified by reduce

content:

application/json:

schema:

type: array

items:

'401' :

\$ref: '\#/components/schemas/Data'

description: Not authorized

' 404 ' :

description: The resource could not be found

/mapreduce/map/ $\{$ name $\}$ :

put:

tags:

- mapreduce

summary: Create or update the map function

description: Create or update the map function

operationId: cloudmesh.mapreduce.map.put

parameters:

- name: name

in: path

required: true

schema: 
NIST Big DATA INTEROPERABILITY FRAMEWORK: Volume 8, REFERENCE ARCHITECTURE INTERFACES

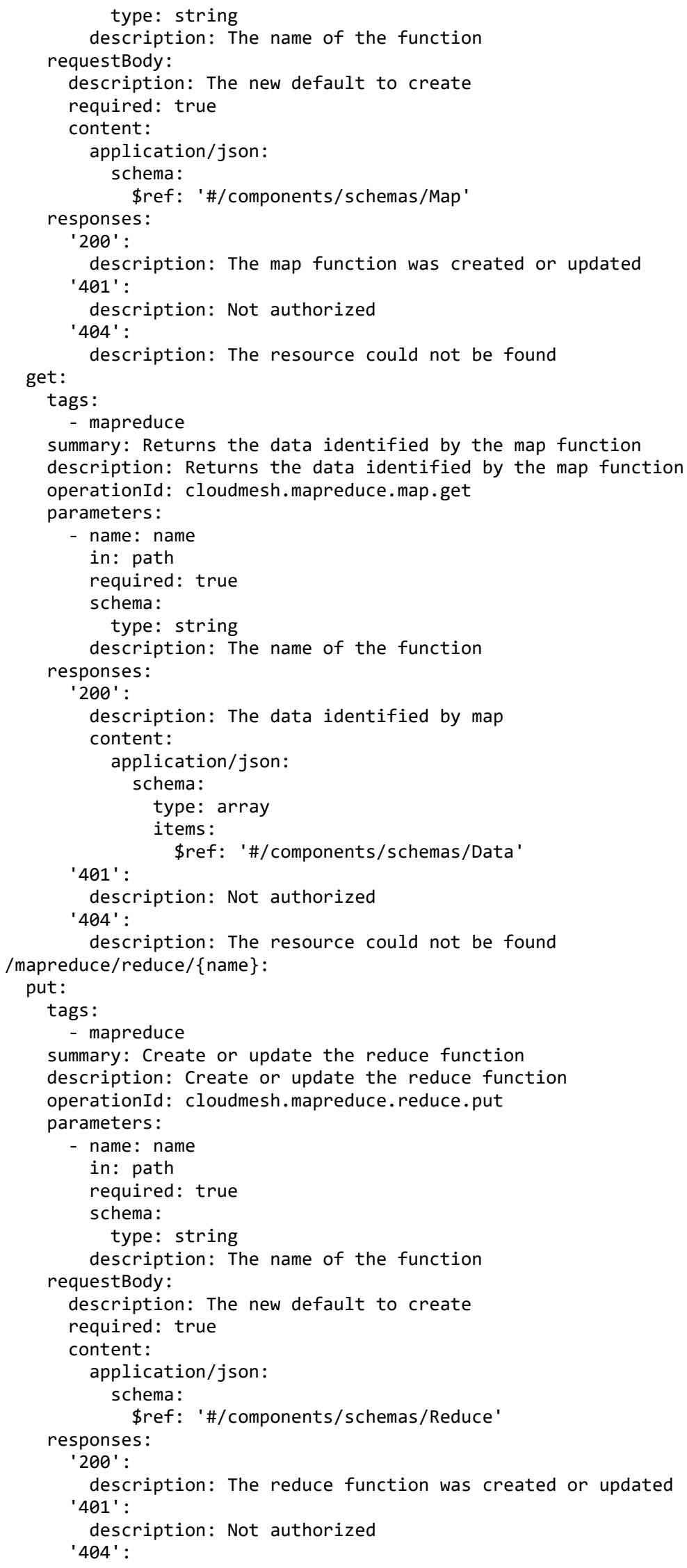


NIST Big DATA INTEROPERABILITY FRAMEWORK: Volume 8, REFERENCE ARCHITECTURE INTERFACES

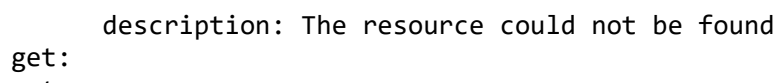




\section{$5450 \quad 4.12$ COMPUTE MANAGEMENT: FUNCTIONS}

\section{$5451 \quad$ 4.12.1 MICROSERVICE}

5452 As part of microservices, a function with parameters that can be invoked has been defined.

$5453 \quad$ 4.12.1.1 Schema Microservice

\begin{tabular}{lrl} 
Property & Type & Description \\
\hline name & string & Name of the microservice \\
endpoint & string & The end point of the microservice \\
function & string & The function the microservice represents \\
description & & The description of the microservice
\end{tabular}

$5454 \quad$ 4.12.1.2 Paths

\begin{tabular}{lll} 
HTTP & Path & Summary \\
\hline get & /microservice & Returns a list of microservicees \\
get & /microservice/\{name $\}$ & Returns the named microservice \\
put & $/$ microservice $/\{$ name $\}$ & Set an microservice \\
delete & $/$ microservice $/$ nname $\}$ & Deletes the named microservice
\end{tabular}

$5455 \quad$ 4.12.1.2.1/microservice

5456 4.12.1.2.1.1 GET/microservice

5457 Returns a list of all microservices

5458 Responses

Code Description Schema

200 The list of microserviceses array[Microservice]

401 Not authorized String

$5459 \quad$ 4.12.1.2.2/microservice/\{name\}

5460 4.12.1.2.2.1 GET/microservice/\{name\}

5461 Returns the named microservice

5462 Responses

\begin{tabular}{lll} 
Code & Description & Schema \\
\hline 200 & Returns the microservice & Microservice \\
401 & Not authorized & String \\
404 & The named microservice could not be found & String
\end{tabular}


NIST Big DATA INTEROPERABILITY FRAMEWORK: Volume 8, REFERENCE ARCHITECTURE INTERFACES

Parameters

\begin{tabular}{lllll} 
Name & Located in & Description & Required & Schema \\
\hline name & path & The name of the microservice & True & String
\end{tabular}

\subsection{PUT /microservice/\{name\}}

5465 Sets the named microservice

5466 Responses

\begin{tabular}{lll} 
Code & Description & Schema \\
\hline 200 & Microservice updated & String \\
400 & Error updating microservice & String \\
401 & Not authorized & String
\end{tabular}

5467 Request Body

Located in Description Required Schema

Body The new microservice to create True

\subsection{DELETE /microservice/\{name\}}

5469 Deletes an microservice by name

5470 Responses

Code Description

Schema

200 Deletion successful

String

400 Error deleting microservice

String

401 Not authorized

String

404 The named microservice could not be found String

Parameters

\begin{tabular}{lllll} 
Name & Located in & Description & Required & Schema \\
\hline name & path & The name of the microservice & True & String
\end{tabular}

\subsubsection{3 microservice.yaml}

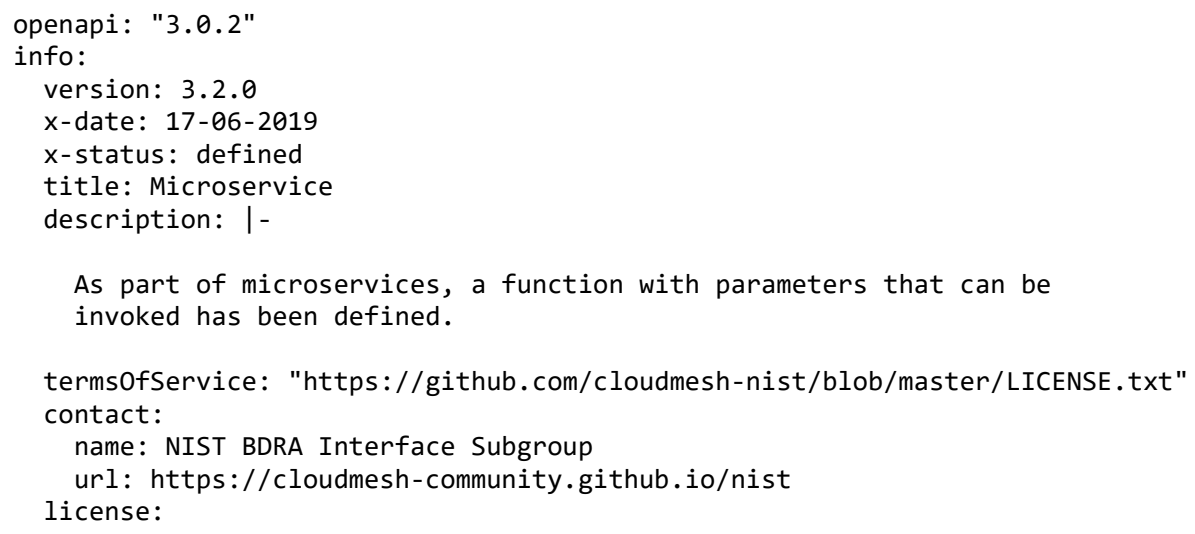


NIST Big DATA INTEROPERABILITY FRAMEWORK: Volume 8, REFERENCE ARCHITECTURE INTERFACES

name: Apache 2.0

url: https://github.com/cloudmesh/cloudmesh-nist/blob/master/LICENSE.txt servers:

- url: /cloudmesh/v3 paths:

/microservice:

get:

tags:

- Microservice

summary: Returns a list of microservicees

description: Returns a list of all microservicees

operationId: cloudmesh.microservice.list responses:

' 200 ' :

description: The list of microserviceses

content:

application/json:

schema:

type: array

items:

' 401 ' :

\$ref: '\#/components/schemas/Microservice'

description: Not authorized

/microservice/ $\{$ name $\}$ :

get :

tags:

- Microservice

summary: Returns the named microservice

description: Returns the named microservice

operationId: cloudmesh.microservice.find_by_name

parameters:

- name: name

in: path

required: true

schema:

type: string

description: The name of the microservice

responses:

'200' :

description: Returns the microservice

content:

application/json:

schema :

' 401 ' :

\$ref: '\#/components/schemas/Microservice'

description: Not authorized

'404' :

description: The named microservice could not be found put:

tags:

- Microservice

summary: Set an microservice

description: Sets the named microservice

operationId: cloudmesh.microservice.add

requestBody:

description: The new microservice to create

required: true

content:

application/json:

schema:

\$ref: '\#/components/schemas/Microservice'

responses:

'200' :

description: Microservice updated

'400' :

description: Error updating microservice

'401' : delete:

description: Not authorized 
NIST Big DATA INTEROPERABILITY FRAMEWORK: VOLUME 8, REFERENCE ARCHITECTURE INTERFACES

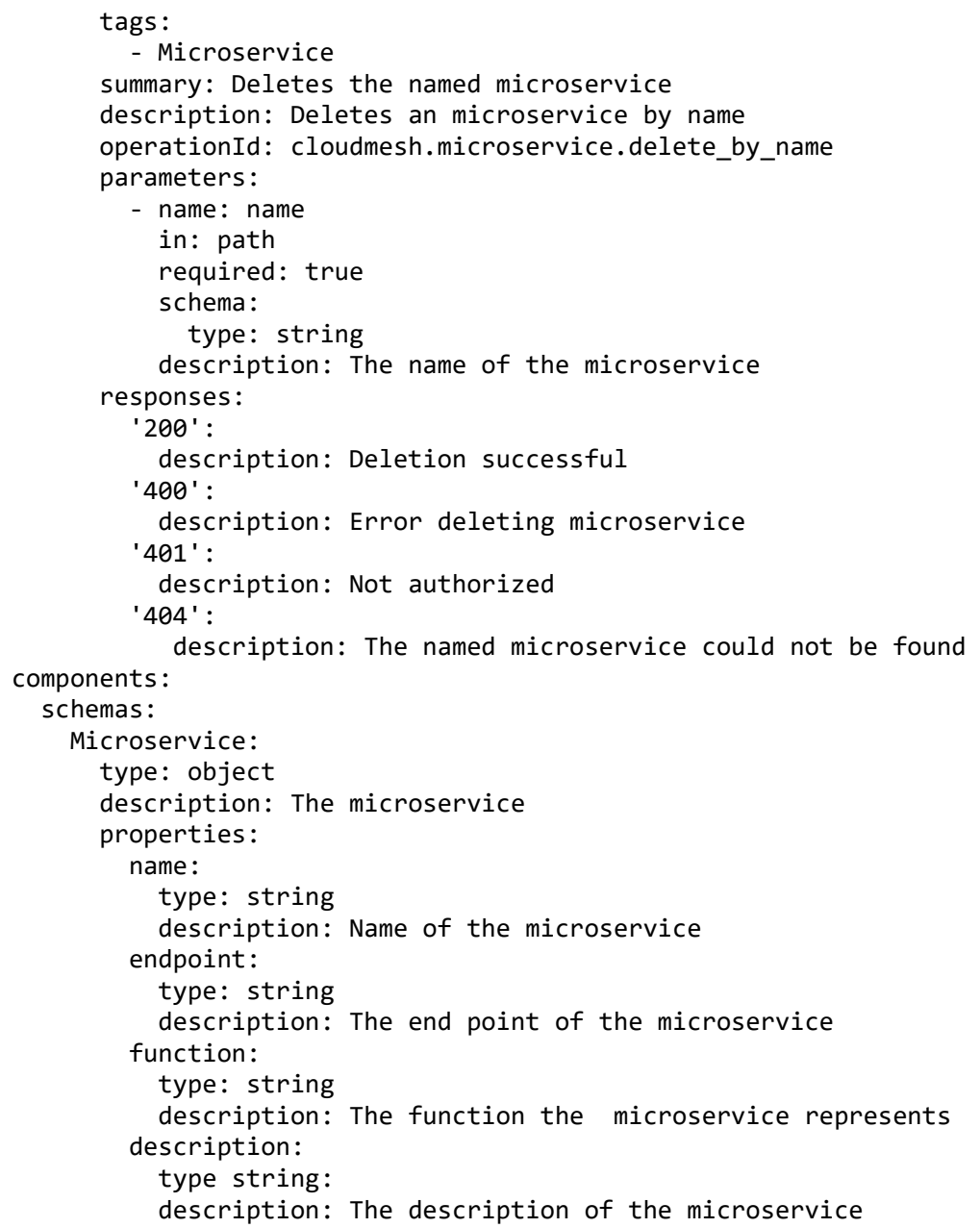

\section{4.13 RESERVATION}

\section{$5598 \quad$ 4.13.1 RESERVATION}

5599 Some services may consume a considerable amount of resources, necessitating the reservation of 5600 resources.

5601 4.13.1.1 Schema Reservation

\begin{tabular}{lll} 
Property & Type & Description \\
\hline name & string & Name of the reservation \\
service & string & The name of the service for which the reservation is applied \\
description & string & The description of the reservation \\
start & string(date) & The start time and date \\
end & string(date) & The end time and date
\end{tabular}


NIST Big DATA INTEROPERABILITY FRAMEWORK: VOLUME 8, REFERENCE ARCHITECTURE INTERFACES

5602

\subsubsection{Paths}

\begin{tabular}{lll} 
HTTP & Path & Summary \\
\hline get & /reservation & Returns a list of reservations \\
get & $/$ reservation $/\{$ name $\}$ & Returns the named reservation \\
put & $/$ reservation $/\{$ name $\}$ & Uploads a reservation to the list of reservations \\
delete & $/$ reservation $/\{$ name $\}$ & Deletes the named reservation
\end{tabular}

5603 4.13.1.2.1/reservation

5604 4.13.1.2.1.1 GET/reservation

5605 Returns a list of all reservations

5606 Responses

\begin{tabular}{lll} 
Code & Description & Schema \\
\hline 200 & The list of reservations & array[Reservation] \\
400 & No Reservations found & String
\end{tabular}

5607 4.13.1.2.2/reservation/\{name\}

5608 4.13.1.2.2.1 GET/reservation/\{name\}

5609 Returns reservation by name

5610 Responses

\begin{tabular}{lll} 
Code & Description & Schema \\
\hline 200 & Returning the information of the reservation & Reservation \\
400 & No reservation found & String \\
401 & Not authorized & String \\
404 & The named reservation could not be found & String
\end{tabular}

5611 Parameters

\begin{tabular}{lllll} 
Name & Located in & Description & Required & Schema \\
\hline name & path & The name of the reservation & True & String
\end{tabular}

5612 4.13.1.2.2.2 PUT /reservation/\{name\}

5613 Uploads a reservation to the list of reservations

5614 Responses

\begin{tabular}{lll} 
Code & Description & Schema \\
\hline 200 & Reservation updated & String \\
400 & Error updating reservation & String
\end{tabular}


NIST Big DATA INTEROPERABILITY FRAMEWORK: VOLUME 8, REFERENCE ARCHITECTURE INTERFACES

Request Body

Located in Description Required Schema

Body The reservation to be uploaded True $\underline{\text { Reservation }}$

5616

\subsection{DELETE /reservation/\{name\}}

5617 Deletes a reservation by name

Responses

Code Description

Schema

200 Deletion successful String

$400 \quad$ No reservation found

String

401 Not authorized

String

404 The named reservation could not be found String

Parameters

\begin{tabular}{lllll} 
Name & Located in & Description & Required & Schema \\
\hline name & path & The name of the reservation & True & String
\end{tabular}

\subsubsection{3 reservation.yaml}

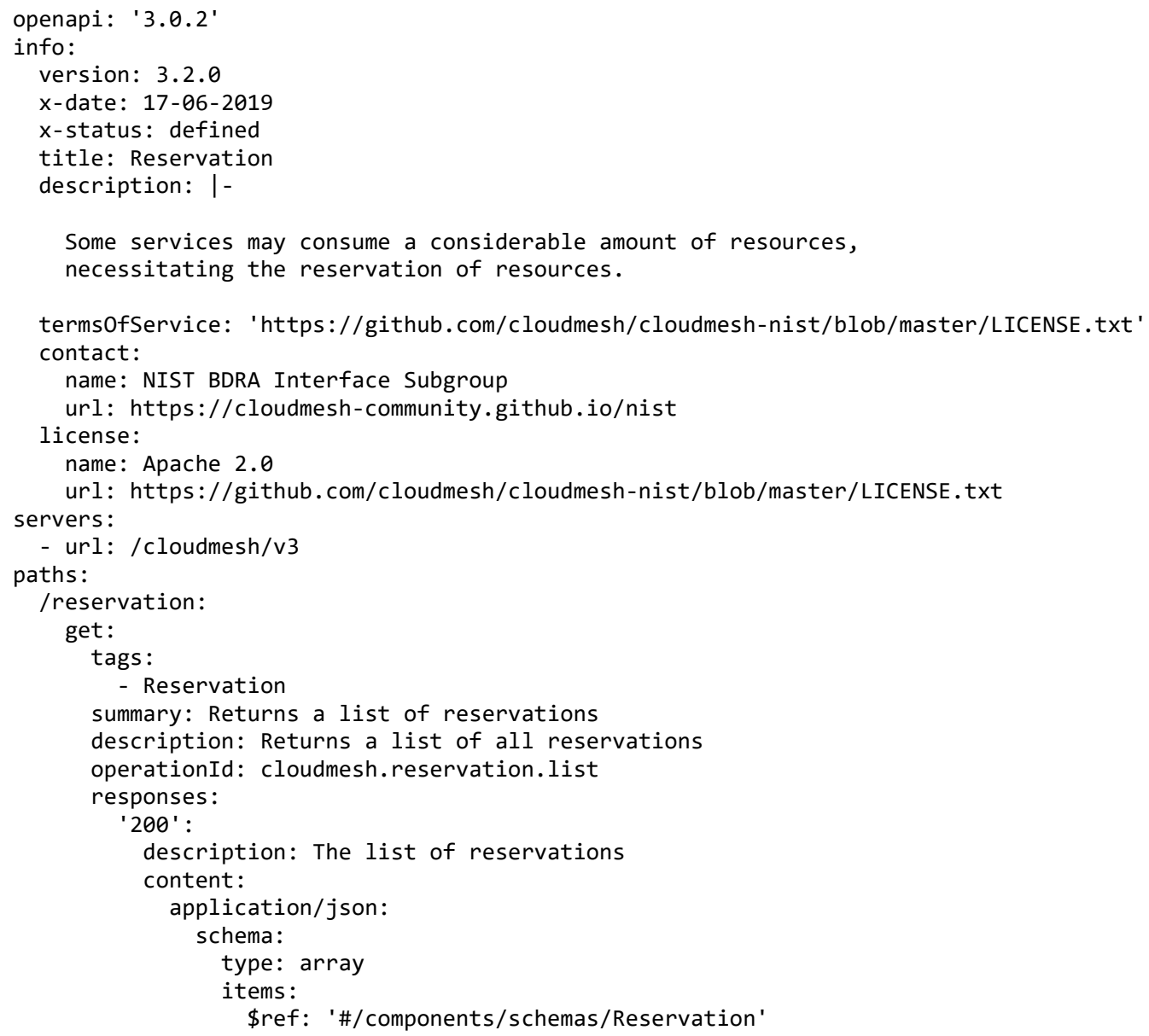


NIST Big DATA INTEROPERABILITY FRAMEWORK: VOLUME 8, REFERENCE ARCHITECTURE INTERFACES

5658

5659

5660

5661

5662

5663

5664

5665

5666

5667

5668

거 5669

5670

5671

5672

5673

5674

5675

5676

5677

5678

5679

5680

5681

5682

5683

5684

5685

5686

5687

5688

5689

5690

5691

5692

5693

5694

5695

5696

5697

5698

5699

5700

5701

5702

5703

5704

5705

5706

5707

5708

5709

5710

5711

5712

5713

5714

5715

5716

5717

5718

5719

5720

5721

5722

5723

5724

5725

5726

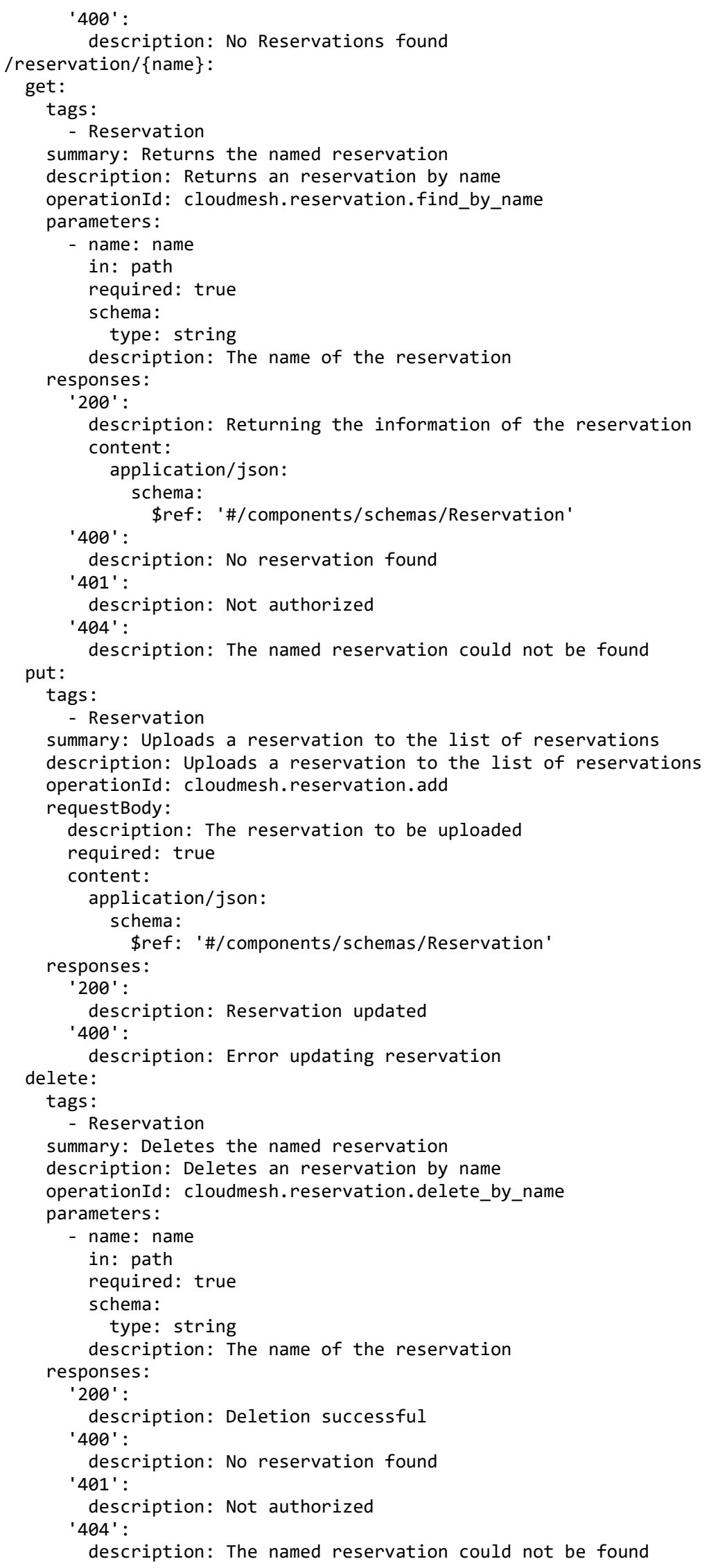




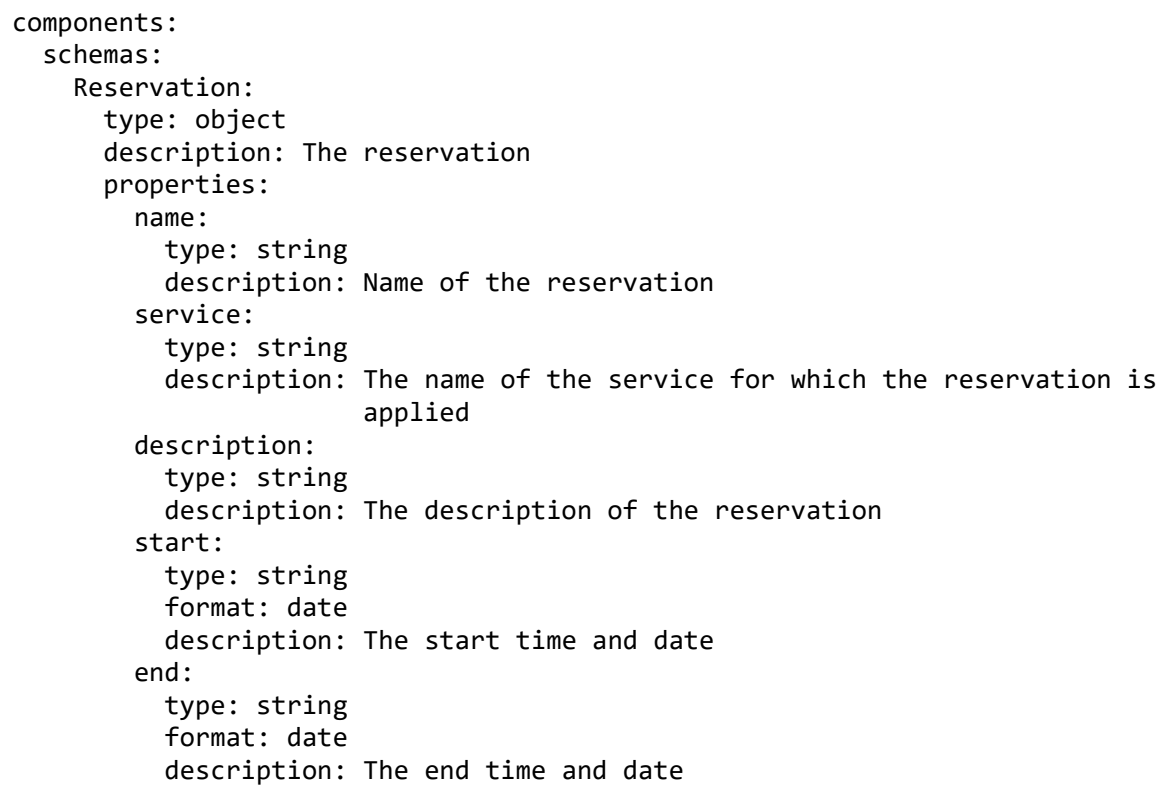

\section{$5752 \quad 4.14 .1 \quad$ STREAM}

5753 The stream object describes a data flow, providing information about the rate and number of items

5754 exchanged while issuing requests to the stream. A stream may return data items in a specific format that is 5755 defined by the stream.

5756 4.14.1.1 Schema Stream

\begin{tabular}{lll} 
Property & Type & Description \\
\hline name & string & Name of the stream \\
format & string & Format of the stream \\
rate & integer & The rate of messages \\
limit & integer & The limit of items send \\
endpoint & string & The endpoint of the stream \\
protocol & string & DThe definition of the protocol used
\end{tabular}

5757 4.14.1.2 Paths

\begin{tabular}{lll} 
HTTP & Path & Summary \\
\hline get & $/$ stream & Returns a list of streams \\
get & $/$ stream $/\{$ name $\}$ & Returns the named stream \\
put & $/$ stream $/\{$ name $\}$ & Set an stream \\
delete & $/$ stream $/\{$ name $\}$ & Deletes the named stream
\end{tabular}




\subsubsection{1/stream}

5759

4.14.1.2.1.1 GET/stream

5760 Returns a list of all streams

5761 Responses

Code Description Schema

200 The list of streams array[Stream]

$400 \quad$ No Stream found String

$401 \quad$ Not authorized String

\section{$5762 \quad 4.14 .1 .2 .2 /$ stream/ $\{$ name $\}$}

\section{4.14.1.2.2.1 GET/stream/\{name\}}

5764 Returns a stream by name

5765 Responses

\begin{tabular}{lll} 
Code & Description & Schema \\
\hline 200 & Returning the information of the stream & $\underline{\text { Stream }}$ \\
401 & Not authorized & String \\
404 & The named stream could not be found & String
\end{tabular}

5766 Parameters

\begin{tabular}{lllll} 
Name & Located in & Description & Required & Schema \\
\hline name & path & The name of the stream & True & String
\end{tabular}

5767 4.14.1.2.2.2 PUT/stream/\{name\}

5768 Sets the named stream

5769 Responses

Code Description Schema

200 Stream updated String

401 Not authorized String

5770 Request Body

Located in Description Required Schema

Body The new stream to create True $\underline{\text { Stream }}$

5771 4.14.1.2.2.3 DELETE /stream/\{name\}

5772 Deletes a stream by name 
NIST Big DATA INTEROPERABILITY FRAMEWORK: Volume 8, REFERENCE ARCHITECTURE INTERFACES

Responses

Code Description

Schema

\begin{tabular}{lll}
\hline 200 & Deletion successful & String \\
401 & Not authorized & String \\
404 & The named stream could not be found & String
\end{tabular}

Parameters

\begin{tabular}{lllll} 
Name & Located in & Description & Required & Schema \\
\hline name & path & The name of the stream & True & String
\end{tabular}

\subsubsection{3 stream.yaml}

openapi: "3.0.2"

info:

version: 3.2 .0

$x$-date: 17-06-2019

$x$-status: defined

title: Stream

description: |-

The stream object describes a data flow, providing information about the rate and number of items exchanged while issuing requests

to the stream. A stream may return data items in a specific format

that is defined by the stream.

termsOfService: "https://github.com/cloudmesh/cloudmesh-nist/blob/master/LICENSE.txt" contact:

name: NIST BDRA Interface Subgroup

url: https://cloudmesh-community.github.io/nist

license:

name: Apache 2.0

url: https://github.com/cloudmesh/cloudmesh-nist/blob/master/LICENSE.txt servers:

- url:/cloudmesh/v3

paths:

/stream:

get:

tags:

- Stream

summary: Returns a list of streams

description: Returns a list of all streams

operationId: cloudmesh.stream.list

responses:

'200' :

description: The list of streams

content:

application/json:

schema:

type: array

items:

' 400 ' :

\$ref: '\#/components/schemas/Stream'

description: No Stream found

' 401 ' :

description: Not authorized

/stream/\{name $\}$ :

get:

tags:

- Stream

summary: Returns the named stream

description: Returns an stream by name

operationId: cloudmesh.stream.find_by_name 
NIST Big DATA INTEROPERABILITY FRAMEWORK: Volume 8, REFERENCE ARCHITECTURE INTERFACES

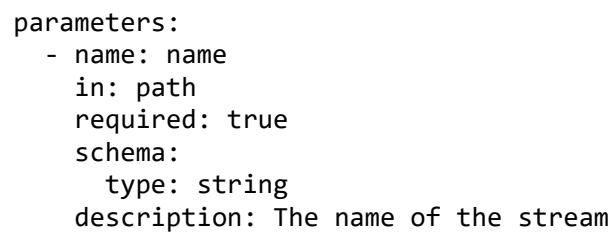


NIST Big DATA INTEROPERABILITY FRAMEWORK: VOLUME 8, REFERENCE ARCHITECTURE INTERFACES

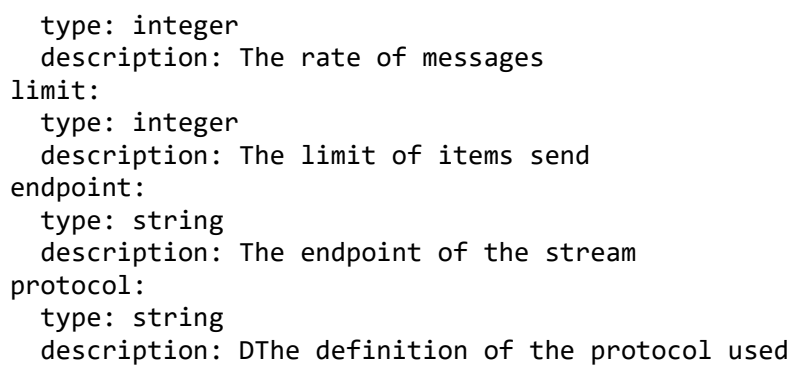

\subsubsection{FILTER}

5907 Filters can operate on a variety of objects and reduce the information received based on a search criterion.

$5908 \quad$ 4.14.2.1 Schema Filter

\begin{tabular}{lll} 
Property & Type & Description \\
\hline name & string & Name of the filter \\
function & string & The function used to filter the data in the stream \\
kind & string & The filter kind or type
\end{tabular}

5909 4.14.2.2 Paths

\begin{tabular}{lll} 
HTTP & Path & Summary \\
\hline get & /filter & Returns a list of filters \\
get & $/$ filter/\{name $\}$ & Returns the named filter \\
put & $/$ filter $/\{$ name $\}$ & Set an filter \\
delete & $/$ filter/\{name $\}$ & Deletes the named filter
\end{tabular}

$5910 \quad$ 4.14.2.2.1/filter

5911 4.14.2.2.1.1 GET /filter

5912 Returns a list of all filters

5913 Responses

\begin{tabular}{lll} 
Code & Description & Schema \\
\hline 200 & The list of filters & array[Filter] \\
401 & Not authorized & String
\end{tabular}

$5914 \quad 4.14 .2 .2 .2 / f i l t e r /\{n a m e\}$

5915 4.14.2.2.2.1 GET/filter/\{name\}

5916 Returns a filter by name 
NIST Big DATA INTEROPERABILITY FRAMEWORK: Volume 8, REFERENCE ARCHITECTURE INTERFACES

5917 Responses

Code Description Schema

200 Returning the information of the filter $\underline{\text { Filter }}$

401 Not authorized String

404 The named filter could not be found String

5918 Parameters

\begin{tabular}{lllll} 
Name & Located in & Description & Required & Schema \\
\hline name & path & The name of the filter & True & String
\end{tabular}

5919 4.14.2.2.2.2 PUT /filter/\{name\}

5920 Sets the named filter

5921 Responses

Code Description Schema

$200 \quad$ Filter updated $\quad$ String

401 Not authorized String

400 Error updating filter String

5922 Request Body

\begin{tabular}{llll} 
Located in & Description & Required & Schema \\
\hline Body & The new filter to create & True & $\underline{\text { Filter }}$
\end{tabular}

5923 4.14.2.2.2.3 DELETE /filter/\{name\}

5924 Deletes a filter by name

5925 Responses

\begin{tabular}{lll} 
Code & Description & Schema \\
\hline 200 & Deletion successful & String \\
401 & Not authorized & String \\
404 & The named filter could not be found & String
\end{tabular}

5926 Parameters

\begin{tabular}{lllll} 
Name & Located in & Description & Required & Schema \\
\hline name & path & The name of the filter & True & String
\end{tabular}

\subsubsection{3 filter.yaml}

openapi: "3.0.2"

info:

version: 3.2 .0

$x$-date: $17-06-2019$

$x$-status: defined

title: Filter

description: |- 
NIST Big DATA INTEROPERABILITY FRAMEWORK: Volume 8, REFERENCE ARCHITECTURE INTERFACES

5936

5937

5938

5939

5940

5941

5942

5943

5944

5945

5946

5947

5948

5949

5950

5951

5952

Filters can operate on a variety of objects and reduce the information received based on a search criterion.

termsOfService: "https://github.com/cloudmesh/cloudmesh-nist/blob/master/LICENSE.txt" contact:

name: NIST BDRA Interface Subgroup

url: https://cloudmesh-community.github.io/nist

license:

name: Apache 2.0

url: https://github.com/cloudmesh/cloudmesh-nist/blob/master/LICENSE.txt servers:

- url: /cloudmesh/v3 paths:

/filter:

get:

tags:

- Filter

summary: Returns a list of filters

description: Returns a list of all filters

operationId: cloudmesh.filter.list

responses:

' 200 ' :

description: The list of filters

content:

application/json:

schema:

type: array

items:

'401' :

\$ref: '\#/components/schemas/Filter'

description: Not authorized

/filter/\{name $\}$ :

get:

tags:

- Filter

summary: Returns the named filter

description: Returns an filter by name

operationId: cloudmesh.filter.find_by_name

parameters:

- name: name

in: path

required: true

schema:

type: string

description: The name of the filter

responses:

'200' :

description: Returning the information of the filter

content:

application/json:

schema:

'401' :

\$ref: '\#/components/schemas/Filter'

description: Not authorized

' 404 ' :

description: The named filter could not be found

put:

tags:

- Filter

summary: Set an filter

description: Sets the named filter

operationId: cloudmesh.filter.add

requestBody:

description: The new filter to create

required: true

content:

application/json:

schema:

\$ref: '\#/components/schemas/Filter' 
NIST Big DATA INTEROPERABILITY FRAMEWORK: VOLUME 8, REFERENCE ARCHITECTURE INTERFACES

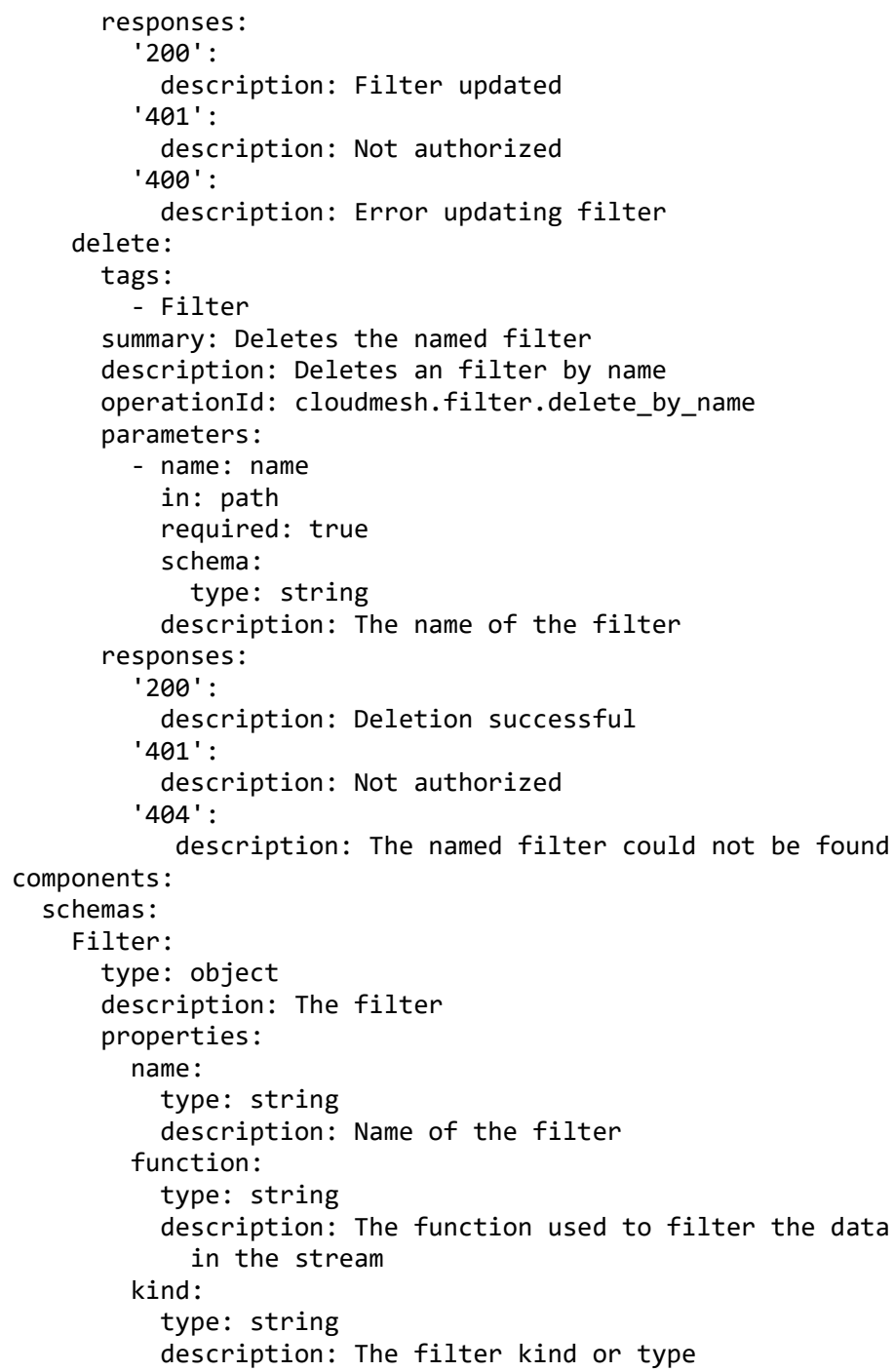

\section{$6048 \quad 4.15$ DEPLOYMENT}

$6049 \quad$ 4.15.1 DEPLOYMENT

6050 A resource to store software deployments. The deployment is formulated in a specification file. To 6051 distinguish the format of the specification file, a string is used that defines the kind of the deployment. In 6052 case the specification uses an external service, an endpoint to the service can be used and the name of the 6053 specification is used to identify the deployment. 


\subsubsection{Schema Deployment}

\begin{tabular}{lll} 
Property & Type & Description \\
\hline name & string & The name of the deployment \\
kind & string & The kind of the deployment \\
specification & string & The specification of the deployment \\
endpoint & string & The location of the deployment service \\
endpointname & string & $\begin{array}{l}\text { In case an endpoint is used, the endpointname is used to uniquely identify the } \\
\text { deployment within the endpoint defined service }\end{array}$
\end{tabular}

\subsubsection{Paths}

\begin{tabular}{lll} 
HTTP & Path & Summary \\
\hline get & /deployment & Returns a list of deployments \\
get & $/$ deployment $/\{$ name $\}$ & Returns the named deployment \\
put & $/$ deployment $/\{$ name $\}$ & Set an deployment \\
delete & $/$ deployment $/\{$ name $\}$ & Deletes the named deployment
\end{tabular}

$6056 \quad 4.15 .1 .2 .1 / d e p l o y m e n t$

6057 4.15.1.2.1.1 GET /deployment

6058 Returns a list of all deployments

6059 Responses

\begin{tabular}{lll} 
Code & Description & Schema \\
\hline 200 & The list of deployments & array[Deployment] \\
401 & Not authorized & String
\end{tabular}

$6060 \quad 4.15 .1 .2 .2 / d e p l o y m e n t /\{$ name $\}$

\section{$6061 \quad$ 4.15.1.2.2.1 GET/deployment/\{name\}}

6062 Returns a deployment by name

6063 Responses

\begin{tabular}{lll} 
Code & Description & Schema \\
\hline 200 & Returning the information of the deployment & Deployment \\
401 & Not authorized & String \\
404 & The named deployment could not be found & String
\end{tabular}

6064 Parameters

\begin{tabular}{lllll} 
Name & Located in & Description & Required & Schema \\
\hline name & path & The name of the deployment & True & String
\end{tabular}


NIST Big DATA INTEROPERABILITY FRAMEWORK: VOLUME 8, REFERENCE ARCHITECTURE INTERFACES

6065

6066

6067

\subsection{PUT /deployment/\{name\}}

Sets the named deployment

Responses

Code Description Schema

200 Deployment updated String

401 Not authorized String

Request Body

Located in Description Required Schema

$\begin{array}{llll}\text { Body } & \text { The new deployment to create } & \text { True } & \text { Deployment }\end{array}$

\subsection{DELETE /deployment/\{name\}}

Deletes a deployment by name

Responses

Code Description Schema

200 Deletion successful String

401 Not authorized String

404 The named deployment could not be found String

Parameters

Name Located in Description Required Schema

name path The name of the deployment True String

\subsubsection{3 deployment.yaml}

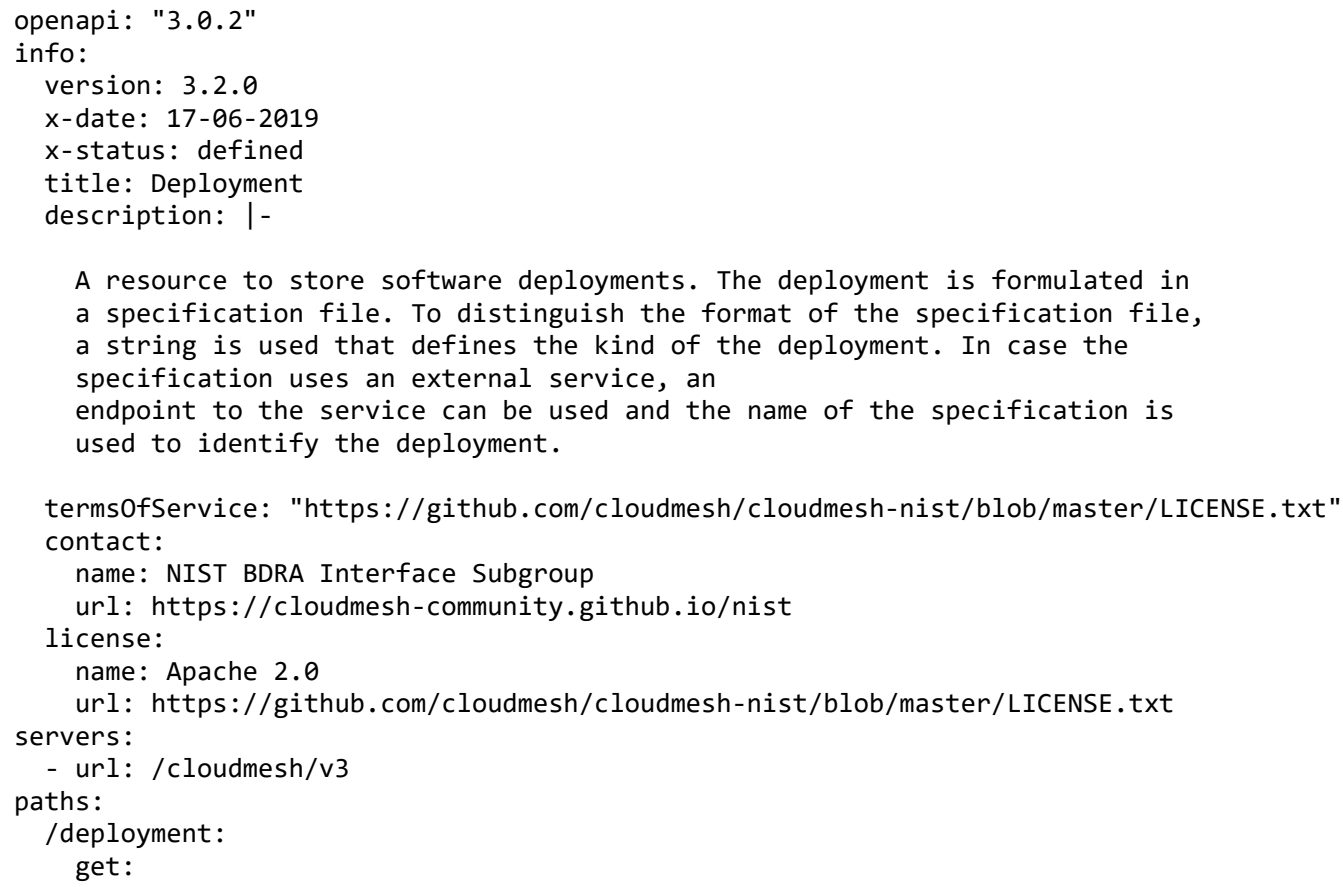


NIST Big DATA INTEROPERABILITY FRAMEWORK: VOLUME 8, REFERENCE ARCHITECTURE INTERFACES

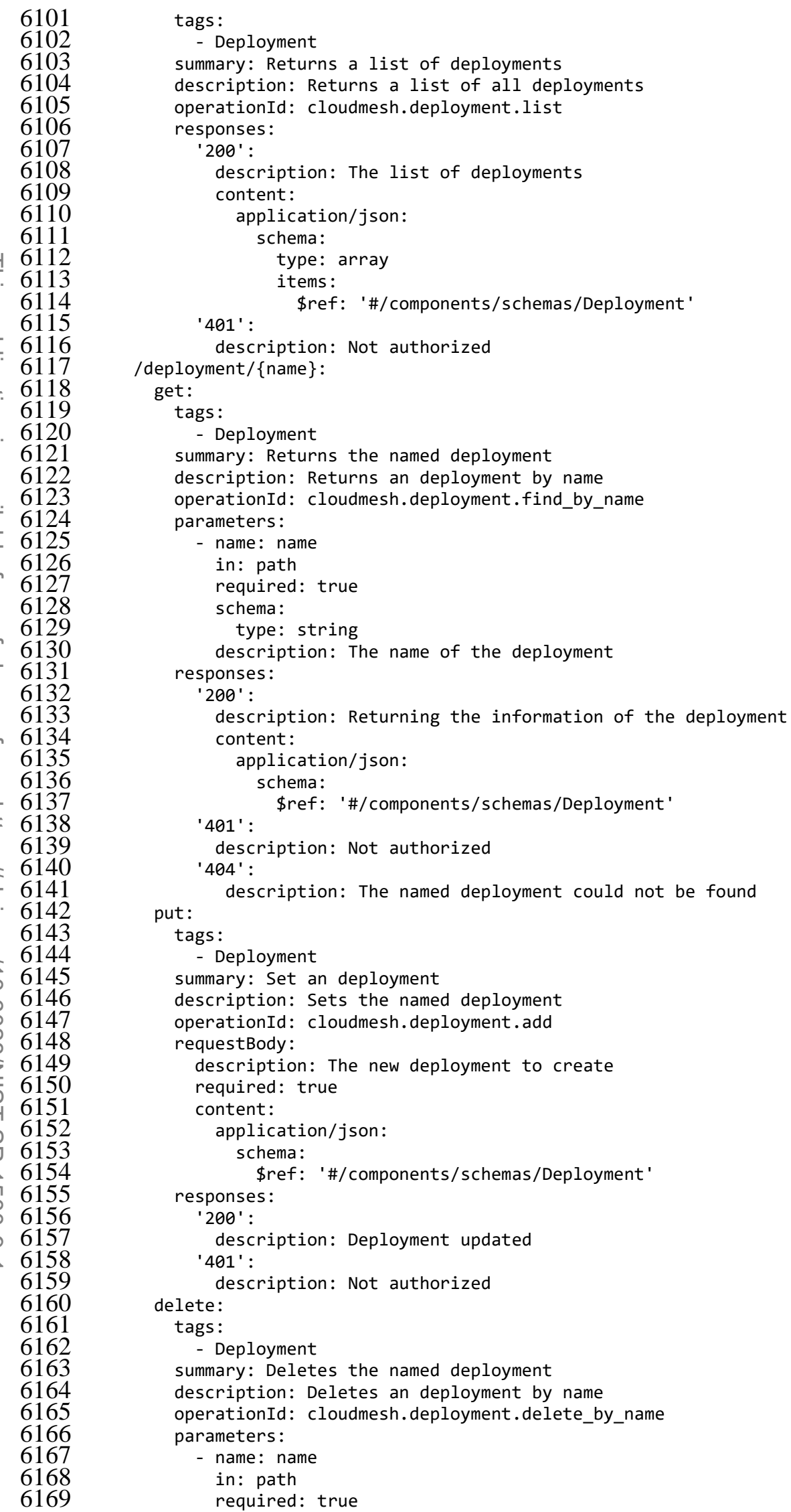


NIST Big DATA INTEROPERABILITY FRAMEWORK: VOLUME 8, REFERENCE ARCHITECTURE INTERFACES

6170

6171

6172

6173

6174

6175

6176

6177

6178

6179

6180

거 6181

그. 6182

6183

ㄷ 6184

흥 6185

त. 6186

․ 6187

후 6188

- 6189

क 6190

¿ 6191

๗. 6192

ज) 6193

드 6194

(D) 6195

त्ञ 6196

(ब) 6197

ㅇ 6198

6199

6200

6201

6202

6203

schema:

type: string

description: The name of the deployment

responses:

'200' :

description: Deletion successful

' 401 ' :

description: Not authorized

'404' :

description: The named deployment could not be found

components:

schemas:

Deployment:

type: object

description: the deployment

properties:

name:

type: string

description: The name of the deployment

kind:

type: string

description: The kind of the deployment

specification:

type: string

description: The specification of the deployment

endpoint:

type: string

description: The location of the deployment service endpointname:

type: string

description: in case an endpoint is used, the endpointname is used

to uniquely identify the deployment within the

endpoint defined service 


\section{Appendix A: Acronyms and Terms}

6205

ACID

6206 API

-1 6207

6208

6209

6210 Container

6211 Cloud Computing

6212

6213

$6214 \mathrm{CPU}$

6215 DevOps

6216

6217 Deployment

6218 DSA

6219 ETL

6220 ELT

6221 FIFO

6222 FPGAs

6223 GPUs

6224 HTTP

6225 HTTPS

6226 Hybrid Cloud

6227

6228

6229

6230

6231

6232

6233

6234

6235

6236

MTU

6237
Atomicity, Consistency, Isolation, Durability

Application Programming Interface

American Standard Code for Information Interchange

Basically Available, Soft state, Eventual consistency

Classless Inter-Domain Routing

See http://csrc.nist.gov/publications/drafts/800-180/sp800-180_draft.pdf

The practice of using a network of remote servers hosted on the Internet to store, manage, and process data, rather than a local server or a personal computer. See http://nvlpubs.nist.gov/nistpubs/Legacy/SP/nistspecialpublication800-145.pdf.

Central Processing Unit

A clipped compound of software DEVelopment and information technology OPerations

The action of installing software on resources

Digital Signature Algorithm

Extract, Transform, Load

Extract, Load, Transform

first-in-first-out

field-programmable gate arrays

graphics processing units

HyperText Transfer Protocol

HTTP Secure

See http://nvlpubs.nist.gov/nistpubs/Legacy/SP/nistspecialpublication800145.pdf.

Internet Control Message Protocol

Internet Protocol

Information Technology Laboratory

last-in-first-out

Media Access Control
Is an approach to build applications based on many smaller modular services. Each module supports a specific goal and uses a simple, well-defined interface to communicate with other sets of services.

maximum transmission unit

NIST Big Data Interoperability Framework 


\begin{tabular}{|c|c|c|}
\hline 6238 & NBD-PWG & NIST Big Data Public Working Group \\
\hline 6239 & NBDRA & NIST Big Data Reference Architecture \\
\hline 6240 & NBDRAI & NIST Big Data Reference Architecture Interface \\
\hline 6241 & NIC & Network Interface Controller \\
\hline 6242 & NIST & National Institute of Standards and Technology \\
\hline 6243 & NON & Network of Nodes \\
\hline 6244 & OS & Operating System \\
\hline 6245 & RAM & Random Access Memory \\
\hline 6246 & REST & REpresentational State Transfer \\
\hline $\begin{array}{l}6247 \\
6248\end{array}$ & Replica & $\begin{array}{l}\text { A duplicate of a file on another resource to avoid costly transfer costs in case of } \\
\text { frequent access. }\end{array}$ \\
\hline 6249 & RSA & Rivest-Shamir-Adleman \\
\hline 6250 & SSH & Secure Shell \\
\hline $\begin{array}{l}6251 \\
6252 \\
6253 \\
6254 \\
6255\end{array}$ & Serverless Computing & $\begin{array}{l}\text { Serverless computing specifies the paradigm of function as a service (FaaS). It is } \\
\text { a cloud computing code execution model in which a cloud provider manages the } \\
\text { function deployment and utilization while clients can utilize them. The charge } \\
\text { model is based on execution of the function rather than the cost to manage and } \\
\text { host the VM or container. }\end{array}$ \\
\hline $\begin{array}{l}6256 \\
6257\end{array}$ & Software Stack & $\begin{array}{l}\text { A set of programs and services that are installed on a resource to support } \\
\text { applications. }\end{array}$ \\
\hline 6258 & TCP & Transmission Control Protocol \\
\hline 6259 & UDP & User Datagram Protocol \\
\hline 6260 & URI & Uniform Resource Identifier \\
\hline $\begin{array}{l}6261 \\
6262\end{array}$ & Virtual File System & $\begin{array}{l}\text { An abstraction layer on top of a distributed physical file system to allow easy } \\
\text { access to the files by the user or application. }\end{array}$ \\
\hline $\begin{array}{l}6263 \\
6264 \\
6265 \\
6266\end{array}$ & VM & $\begin{array}{l}\text { Virtual Machine. A virtual machine is a software computer that, like a physical } \\
\text { computer, runs an operating system and applications. The VM is composed of a } \\
\text { set of specification and configuration files and is backed by the physical } \\
\text { resources of a host. }\end{array}$ \\
\hline $\begin{array}{l}6267 \\
6268 \\
6269 \\
6270 \\
6271 \\
6272\end{array}$ & Virtual Cluster & $\begin{array}{l}\text { A virtual cluster is a software cluster that integrate either VMs, containers, or } \\
\text { physical resources into an agglomeration of compute resources. A virtual cluster } \\
\text { allows users to authenticate and authorize to the virtual compute nodes to utilize } \\
\text { them for calculations. Optional high-level services that can be deployed on a } \\
\text { virtual cluster may simplify interaction with the virtual cluster or provide higher- } \\
\text { level services. }\end{array}$ \\
\hline 6273 & Workflow & The sequence of processes or tasks \\
\hline 6274 & WWW & World Wide Web \\
\hline 6275 & & \\
\hline & & \\
\hline
\end{tabular}




\section{Appendix B: Bibliography}

[1] W. Chang, “NIST Big Data Public Working Group (NBD-PWG),” 2018. [Online]. Available: http://bigdatawg.nist.gov/home.php. [Accessed: 03-Apr-2018]

[2] W. L. Chang (Co-Chair), N. Grady (Subgroup Co-chair), and NIST Big Data Public Working Group, “NIST Big Data Interoperability Framework: Volume 1, Big Data Definitions (NIST SP 1500-1 VERSION 3),” Gaithersburg MD, Sep. 2019 [Online]. Available: https://doi.org/10.6028/NIST.SP.1500-1r2

[3] W. L. Chang (Co-Chair), N. Grady (Subgroup Co-chair), and NIST Big Data Public Working Group, “NIST Big Data Interoperability Framework: Volume 2, Big Data Taxonomies (NIST SP 1500-2 VERSION 3),” Gaithersburg, MD, Sep. 2019 [Online]. Available: https://doi.org/10.6028/NIST.SP.1500-2r2

[4] W. L. Chang (Co-Chair), G. Fox (Subgroup Co-chair), and NIST Big Data Public Working Group, "NIST Big Data Interoperability Framework: Volume 3, Big Data Use Cases and General Requirements (NIST SP 1500-3 VERSION 3),” Gaithersburg, MD, Sep. 2019 [Online]. Available: https://doi.org/10.6028/NIST.SP.1500-3r2

[5] W. L. Chang (Co-Chair), A. Roy (Subgroup Co-chair), M. Underwood (Subgroup Co-chair), and NIST Big Data Public Working Group, "NIST Big Data Interoperability Framework: Volume 4, Big Data Security and Privacy (NIST SP 1500-4 VERSION 3),” Gaithersburg, MD, Sep. 2019 [Online]. Available: https://doi.org/10.6028/NIST.SP.1500-4r2

[6] W. L. Chang (Co-Chair), S. Mishra (Editor), and NIST Big Data Public Working Group, “NIST Big Data Interoperability Framework: Volume 5, Big Data Architectures White Paper Survey (NIST SP 1500-5 VERSION 1),” Sep. 2015.

[7] W. L. Chang (Co-Chair), D. Boyd (Subgroup Co-chair), O. Levin (Version 1 Subgroup Co-Chair), and NIST Big Data Public Working Group, "NIST Big Data Interoperability Framework: Volume 6, Big Data Reference Architecture (NIST SP 1500-6 VERSION 3),” Gaithersburg MD, Sep. 2019 [Online]. Available: https://doi.org/10.6028/NIST.SP.1500-6r2

[8] W. L. Chang (Co-Chair), R. Reinsch (Subgroup Co-chair), D. Boyd (Version 1 Subgroup Cochair), C. Buffington (Version 1 Subgroup Co-chair), and NIST Big Data Public Working Group, "NIST Big Data Interoperability Framework: Volume 7, Big Data Standards Roadmap (NIST SP 1500-7 VERSION 3),” Gaithersburg, MD, Sep. 2019 [Online]. Available: https://doi.org/10.6028/NIST.SP.1500-7r2

[9] W. L. Chang (Co-Chair), R. Reinsch (Subgroup Co-chair), C. Austin (Editor), and NIST Big Data Public Working Group, "NIST Big Data Interoperability Framework: Volume 9, Adoption and Modernization (NIST SP 1500-10 VERSION 2),” Gaithersburg, MD, Sep. 2019 [Online]. Available: https://doi.org/10.6028/NIST.SP.1500-10r1 
NIST Big DATA INTEROPERABILITY FRAMEWORK: VOLUME 8, REFERENCE ARCHITECTURE INTERFACES

[10] T. White House Office of Science and Technology Policy, “Big Data is a Big Deal,” OSTP Blog, 2012. [Online]. Available: http://www.whitehouse.gov/blog/2012/03/29/big-data-big-deal. [Accessed: 21-Feb-2014]

[11] W. L. Chang (Co-Chair), N. Grady (Subgroup Co-chair), and NIST Big Data Public Working Group, “NIST Big Data Interoperability Framework: Volume 1, Big Data Definitions (NIST SP 1500-1 VERSION 2),” Jun. 2018 [Online]. Available: https://doi.org/10.6028/NIST.SP.1500-1r1

[12] W. L. Chang (Co-Chair) and G. Fox (Subgroup Co-chair), “NIST Big Data Interoperability Framework: Volume 3, Big Data Use Cases and General Requirements (NIST SP 1500-3 VERSION 2),” Jun. 2018.

[13] W. L. Chang (Co-Chair), A. Roy (Subgroup Co-chair), M. Underwood (Subgroup Co-chair), and NIST Big Data Public Working Group, “NIST Big Data Interoperability Framework: Volume 4, Big Data Security and Privacy (NIST SP 1500-4 VERSION 2),” Jun. 2018.

[14] W. L. Chang (Co-Chair), D. Boyd (Subgroup Co-chair), and NIST Big Data Public Working Group, "NIST Big Data Interoperability Framework: Volume 6, Big Data Reference Architecture (NIST SP 1500-6 VERSION 2),” Jun. 2018.

[15] W. L. Chang (Co-Chair), R. Reinsch (Subgroup Co-chair), and NIST Big Data Public Working Group, “NIST Big Data Interoperability Framework: Volume 7, Big Data Standards Roadmap (NIST SP 1500-7 VERSION 2),” Jun. 2018.

[16] W. L. Chang (Co-Chair), G. von Laszewski (Editor), and NIST Big Data Public Working Group, “NIST Big Data Interoperability Framework: Volume 8, Big Data Reference Architecture Interfaces (NIST SP 1500-9 VERSION 1),” Jun. 2018.

[17] W. L. Chang (Co-Chair), R. Reinsch (Subgroup Co-chair), and NIST Big Data Public Working Group, "NIST Big Data Interoperability Framework: Volume 9, Adoption and Modernization (NIST SP 1500-10 VERSION 1),” Jun. 2018.

[18] Department of Defense, “The DoDAF Architecture Framework Version 2.02,” Apr. 2010 [Online]. Available: https://dodcio.defense.gov/library/dod-architecture-framework/

[19] R. Fielding (Editor) and J. Reschke (Editor), "Hypertext Transfer Protocol (HTTP/1.1): Semantics and Content,” Internet Engineering Task Force (IETF), Jun-2014. [Online]. Available: https://tools.ietf.org/html/rfc7231

[20] G. Klyne (Editor) and C. Newman, "Date and Time on the Internet: Timestamps,” Internet Engineering Task Force, Network Working Group, Jul-2002. [Online]. Available: https://xml2rfc.tools.ietf.org/public/rfc/html/rfc3339.html\#anchor14

[21] Internet2 Middleware Architecture Committee for Education Directory Working Group (MACEDir), “eduPerson Object Class Specification (201602),” 2016. [Online]. Available: http://software.internet2.edu/eduperson/internet2-mace-dir-eduperson-201602.html. [Accessed: 15-Nov-2017] 